\title{
Glossary of Uranium-
}

and Thorium-Bearing

Minerals

FOURTH EDITION

GEOLOGICAL SURVEY BULLETIN 1250 


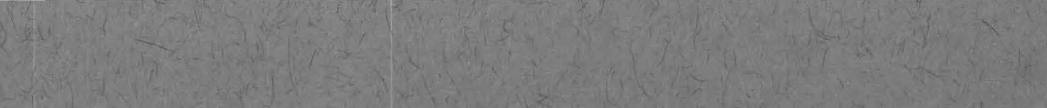

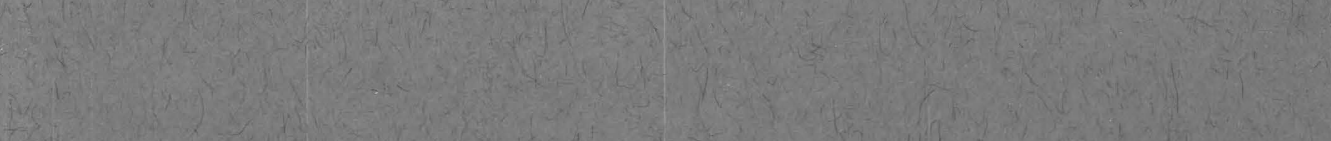

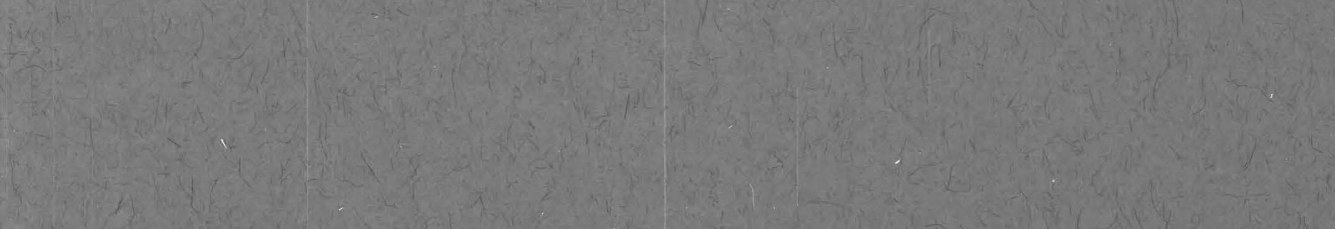

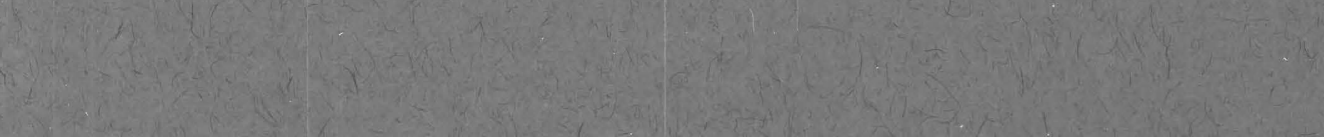

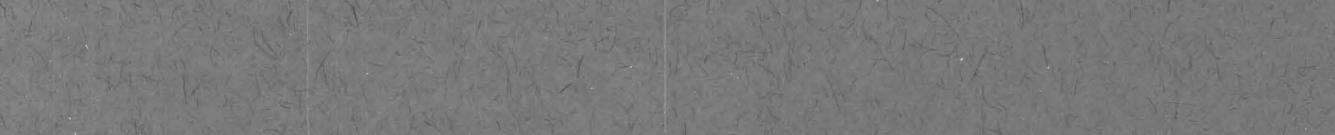

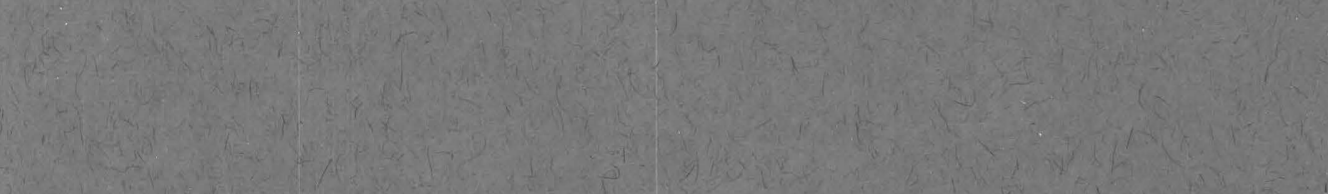

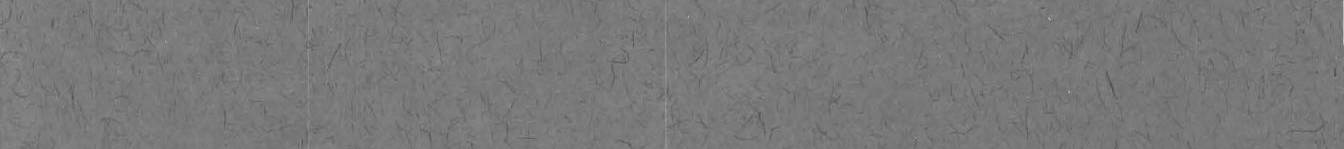

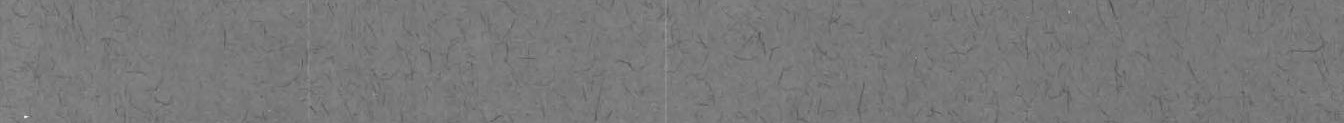

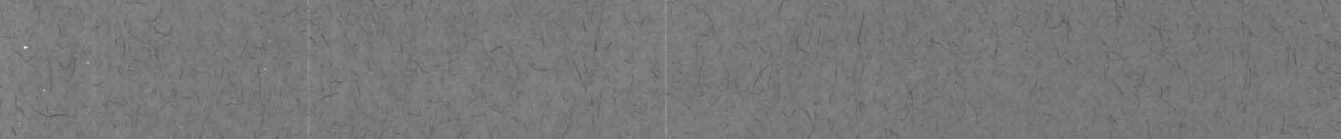

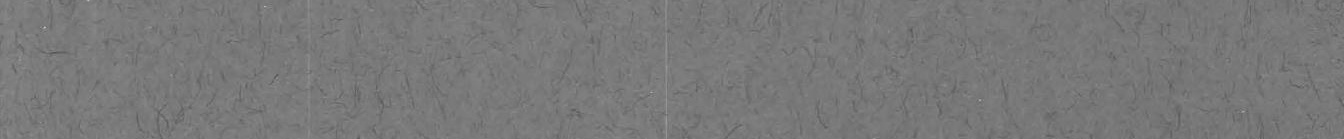

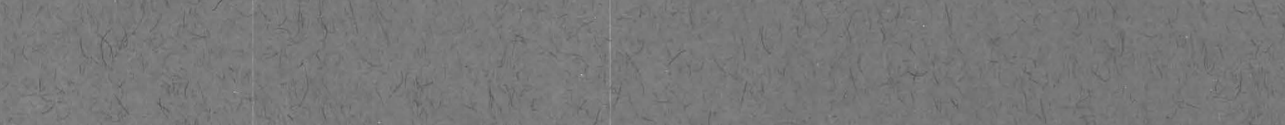

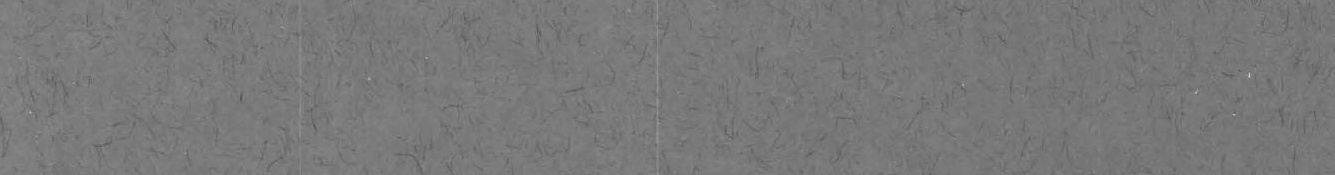
ate.

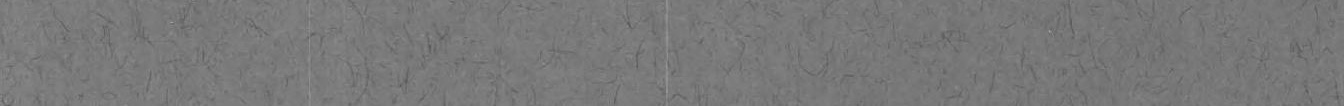

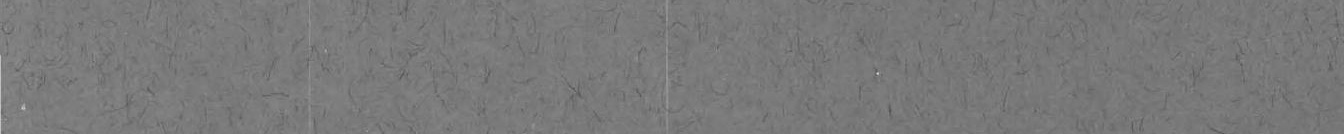

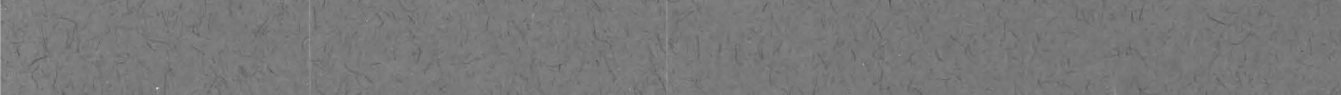
- 


\section{Glossary of Uranium-}

\section{and Thorium-Bearing}

\section{Minerals}

\section{OURTH EDITION}

JUDITH W. FRONDEL, MICHAEL FLEISCHER, and ROBERT S. JONES

E O L G I C A L S U R V E Y B L L E T I N 1250

4 list of uranium-and thorium-containing ninerals, with data on composition, type foccurrence, chemical classification, ind synonymy

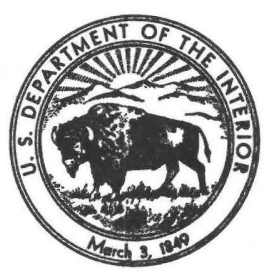


UNITED STATES DEPARTMENT OF THE INTERIOR

STEWART L. UDALL, Secretary

\section{GEOLOGICAL SURVEY}

William T. Pecora, Director

Library of Congress catalog-card No. GS 67-278

For sale by the Superintendent of Documents, U.S. Government Printing Office Washington, D.C. 20402 - Price 30 cents (paper cover) 


\section{CONTENTS}

Page

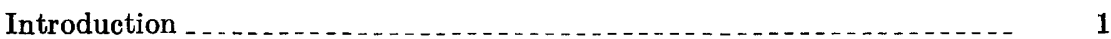

Chemical classification of the uranium and thorium minerals........ 5

A. Uranium and thorium minerals . . 10

B. Minerals with minor amounts of uranium and thorium 50

C. Minerals reported to contain uranium and thorium minerals as impurities or intergrowths ...... 61

Index

III 



\title{
GLOSSARY OF URANIUM- AND THORIUM-BEARING MINERALS
}

\author{
FOURTH EDITION
}

By Judith W. Frondel, Michael Fleischer, and Robert S. Jones

\section{INTRODUCTION}

The first edition of this work was published as U.S. Geological Survey Circular 74 in April 1950, the second edition as Circular 194 in February 1952, and the third edition in 1955 as U. S. Geological Survey Bulletin 1009-F. Research on radioactive minerals has grown in volume and scope, and this fourth edition, with its numerous additions, revisions, and corrections, brings the glossary up to date as of December 1966.

The compilation of this glossary is part of a continuing systematic survey of data on uranium and thorium minerals and on minerals that contain small amounts of uranium or thorium. This survey consists of collecting authoritative chemical, optical, and X-ray diffraction data from the literature and of adding to these data-where inadequateby further laboratory research. The results obtained are published from time to time as separate papers, and a comprehensive volume on the mineralogy of uranium and thorium appeared in 1958 as U.S. Geological Survey Bulletin 1064.

The glossary is divided into three sections: A, minerals that contain uranium or thorium as a major constituent; $B$, minerals that contain minor amounts of uranium and thorium; and $\mathrm{C}$, minerals that probably do not contain uranium or thorium, but which have been reported to contain impurities or intergrowths of uranium or thorium minerals. Many of the minerals listed are highly variable in composition, some varieties containing much, some little uranium or thorium. The known range of composition is listed for each, but the variability causes the division of species between A and B to be somewhat arbitrary. In the first and second editions of this glossary, a fourth group of nearly 40 minerals was listed as "Minerals That Should Be Tested for Uranium and Thorium"; these minerals had not been reported to contain uranium or thorium, but, on crystal-chemical grounds, they might be expected to show the presence of uranium or thorium if investigated 
by modern methods. This list is not included in the present edition, but many of those minerals have been added to section $B$ as a result of additional studies.

In recent years careful studies have shown that many rock-forming minerals contain a few tenths of a part per million to a few parts per million of uranium and thorium. Data on these are not included; a summary is given by J. A. S. Adams, J. K. Osmond, and J. J. W. Rogers, "The Geochemistry of Thorium and Uranium." 1

It is hoped that this glossary will help to alleviate the confusion that obscures the nomenclature of the uranium and thorium minerals. For many of them, the confusion will remain until more thorough research is done. Some species may always be subject to question because their type specimens have been lost, and existing specimens bearing the species name have been found to be other minerals. For instance, in section A, uranochalcite (p. 45). The synonomy has been given for each species, but it is hoped that the many synonyms that serve no useful purpose will be abandoned. Identities and group relations are indicated. A chemical classification of the uranium and thorium minerals has been included, and very brief statements of the color of the minerals and the type of occurrence have been added.

It is beyond the scope of this publication to describe the minerals that are listed or to include determinative tables. Those interested are referred to the more comprehensive volumes listed below; and especially to "Systematic Mineralogy of Uranium and Thorium," by Clifford Frondel, U.S. Geol. Survey Bull. 1064, 400 p. (1958). For most minerals, the present publication generally gives references to recent papers or to abstracts of them in the American Mineralogist, or to standard reference books, especially to Dana's "System of Mineralogy." 2

Other major works on uranium and thorium are:

Angelelli, Victorio, 1958, Los minerales de uranio; sus yacimientos prospección: Argentia, Com. Nac. de Energía Atómica, Dept. Geología y Minería, 160 p. Butschkowskyj, Miguel, 1958, Minerales radioactivos: [Buenos Aires] Inst. Nac. Invest. Cienc. Nat. y Mus. Argentino Cienc. Nat. "Bernardino Rivadavia" Rev. Ser. Cienc. geol., v. 4, no. 3, p. 293-567.

Chervet, J., and Branche, Georges, 1955, Contribution à l'étude des minéraux secondaires d'uranium français: Sciences de la Terre, v. 3, nos. 1 and 2, p. 3-192.

\footnotetext{
1 In Ahrens, L. H., and others, eds., 1959, Physics and chemistry of the earth, v. 3: London, Pergamon Press, p. 298-348.

6th edition, by E. S. Dana, New York, John Wiley \& Sons (1892) (cited as Dana VI); 7th edition by Charles Palache, Harry Berman, and Clifford Frondel, v. I (1944), v. II (1951), New York John Wiley \& Sons (cited as Dana VII).
} 
Getseva, R. V., and Savel'eva, K. I., 1956, Rukovodstov po opredeleniyu uranovykh mineralov [Handbook for identification of uranium minerals]: Moscow, Gosudar. Nauch.-Tekh. Izd. Lit. Geol. i Okhrane Nedr, 260 p.

Heinrich, E. W., 1958, Mineralogy and geology of radioactive raw materials: New York, McGraw-Hill Book Co., 654 p.

Nininger, R. D., 1956, Minerals for atomic energy: 2d ed., New York, D. Van Nostrand Co., 399 p.

Roubault, Marcel, 1958, Géologie de l'uranium: Paris, Masson et Cie, $462 \mathrm{p}$.

Soboleva, M. V., and Pudovkina, I. A., 1957, Mineraly urana, spravochnik [Handbook of uranium minerals]: Moscow, Gosudar. Nauch.-Tekh. Izd. Lit. Geol. i Okhrane Nedr, 409 p.

The index of mineral names 534 entries. These represent 260 named species: 185 in section $A, 55$ in section $B$, and 20 in section $C$. Often the question is asked, "How many uranium and thorium minerals are there?" If section A is used as the criterion, there are about 185 such minerals to which 300 names have been applied.

We are indebted to many persons for suggestions and for pointing out errors in the previous editions, and we hope that readers will continue to inform us of any errors, omissions, or additional data. 



\section{CHEMICAL CLASSIFICATION OF THE URANIUM AND THORIUM MINERALS}

\section{Arsenates:}

Abernathyite $\mathrm{K}_{2}\left(\mathrm{UO}_{2}\right)_{2}\left(\mathrm{AsO}_{4}\right)_{2} \cdot 6 \mathrm{H}_{2} \mathrm{O}$

Arsenuranylite $\mathrm{Ca}\left(\mathrm{UO}_{2}\right)_{4}\left(\mathrm{AsO}_{4}\right)_{2}(\mathrm{OH})_{4} \cdot 6 \mathrm{H}_{2} \mathrm{O}$

Hallimondite $\mathrm{Pb}_{2}\left(\mathrm{UO}_{2}\right)\left(\mathrm{AsO}_{4}\right)_{2}$

Heinrichite $\mathrm{Ba}\left(\mathrm{UO}_{2}\right)_{2}\left(\mathrm{AsO}_{4}\right)_{2} \cdot 10-12 \mathrm{H}_{2} \mathrm{O}$

Hügelite $\mathrm{Pb}_{2}\left(\mathrm{UO}_{2}\right)_{3}\left(\mathrm{AsO}_{4}\right)_{2}(\mathrm{OH})_{4} \cdot 3 \mathrm{H}_{2} \mathrm{O}$

Kahlerite $\mathrm{Fe}\left(\mathrm{UO}_{2}\right)_{2}\left(\mathrm{AsO}_{4}\right)_{2} \cdot n \mathrm{H}_{2} \mathrm{O}$

Metaheinrichite $\mathrm{Ba}\left(\mathrm{UO}_{2}\right)_{2}\left(\mathrm{AsO}_{4}\right)_{2} \cdot 8 \mathrm{H}_{2} \mathrm{O}$

Metakahlerite $\mathrm{Fe}\left(\mathrm{UO}_{2}\right)_{2}\left(\mathrm{AsO}_{4}\right)_{2} \cdot 8 \mathrm{H}_{2} \mathrm{O}$

Metakirchheimerite $\mathrm{Co}\left(\mathrm{UO}_{2}\right)_{2}\left(\mathrm{AsO}_{4}\right)_{2} \cdot 8 \mathrm{H}_{2} \mathrm{O}$

Metanovacekite $\mathrm{Mg}\left(\mathrm{UO}_{2}\right)_{2}\left(\mathrm{AsO}_{4}\right)_{2} \cdot 4 \mathrm{H}_{2} \mathrm{O}$

Meta-uranospinite $\mathrm{Ca}\left(\mathrm{UO}_{2}\right)_{2}\left(\mathrm{AsO}_{4}\right)_{2} \cdot 8 \mathrm{H}_{2} \mathrm{O}$

Metazeunerite $\mathrm{Cu}\left(\mathrm{UO}_{2}\right)_{2}\left(\mathrm{AsO}_{4}\right)_{2} \cdot 8 \mathrm{H}_{2} \mathrm{O}$

Novacekite $\mathrm{Mg}\left(\mathrm{UO}_{2}\right)_{2}\left(\mathrm{AsO}_{4}\right)_{2} \cdot 8-10 \mathrm{H}_{2} \mathrm{O}$

Paulite $\mathrm{HAl}\left(\mathrm{UO}_{2}\right)_{4}\left(\mathrm{AsO}_{4}\right)_{4} \cdot 16 \mathrm{H}_{2} \mathrm{O}(?)$

Sodium uranospinite $\left(\mathrm{Na}_{2}, \mathrm{Ca}\right)\left(\mathrm{UO}_{2}\right)_{2}\left[(\mathrm{As}, \mathrm{P}) \mathrm{O}_{4}\right]_{2} \cdot 5 \mathrm{H}_{2} \mathrm{O}$

Troegerite $\mathrm{H}_{2}\left(\mathrm{UO}_{2}\right)_{2}\left(\mathrm{AsO}_{4}\right)_{2} \cdot 8 \mathrm{H}_{2} \mathrm{O}$

Uranospathite $\mathrm{Cu}\left(\mathrm{UO}_{2}\right)_{2}\left(\mathrm{AsO}_{4}, \mathrm{PO}_{4}\right)_{2} \cdot 11 \mathrm{H}_{2} \mathrm{O}(?)$

Uranospinite $\mathrm{Ca}\left(\mathrm{UO}_{2}\right)_{2}\left(\mathrm{AsO}_{4}\right)_{2} \cdot 10 \mathrm{H}_{2} \mathrm{O}$

Walpurgite $\mathrm{Bi}_{4}\left(\mathrm{UO}_{2}\right)\left(\mathrm{AsO}_{4}\right)_{2} \mathrm{O}_{4} \cdot 3 \mathrm{H}_{2} \mathrm{O}$

Zeunerite $\mathrm{Cu}\left(\mathrm{UO}_{2}\right)_{2}\left(\mathrm{AsO}_{4}\right)_{2} \cdot 10-12 \mathrm{H}_{2} \mathrm{O}$

See also Unnamed minerals, p. 43-44.

\section{Carbonates:}

Andersonite $\mathrm{Na}_{2} \mathrm{Ca}\left(\mathrm{UO}_{2}\right)\left(\mathrm{CO}_{3}\right)_{3} \cdot 6 \mathrm{H}_{2} \mathrm{O}$

Bayleyite $\mathrm{Mg}_{2}\left(\mathrm{UO}_{2}\right)\left(\mathrm{CO}_{3}\right)_{3} \cdot 18 \mathrm{H}_{2} \mathrm{O}$

Liebigite $\mathrm{Ca}_{2}\left(\mathrm{UO}_{2}\right)\left(\mathrm{CO}_{3}\right)_{3} \cdot 10 \mathrm{H}_{2} \mathrm{O}$

Mackelveyite Near $\mathrm{Na}_{2} \mathrm{Ba} a_{4} \mathrm{Ca}(\mathrm{Y}, \mathrm{U})_{2}\left(\mathrm{CO}_{3}\right)_{0} \cdot 5 \mathrm{H}_{2} \mathrm{O}$

Metazellerite $\mathrm{Ca}\left(\mathrm{UO}_{2}\right)\left(\mathrm{CO}_{3}\right)_{2} \cdot 3 \mathrm{H}_{2} \mathrm{O}$

Rabbittite $\mathrm{Ca}_{3} \mathrm{Mg}_{3}\left(\mathrm{UO}_{2}\right)_{2}\left(\mathrm{CO}_{3}\right)_{6}(\mathrm{OH})_{4} \cdot 18 \mathrm{H}_{2} \mathrm{O}$

Rutherfordine $\left(\mathrm{UO}_{2}\right) \mathrm{CO}_{3}$

Schroeckingerite $\mathrm{NaCa}_{3}\left(\mathrm{UO}_{2}\right)\left(\mathrm{CO}_{3}\right)_{3}\left(\mathrm{SO}_{4}\right) \mathrm{F} \cdot 10 \mathrm{H}_{2} \mathrm{O}$

Sharpite $\left(\mathrm{UO}_{2}\right)\left(\mathrm{CO}_{3}\right) \cdot \mathrm{H}_{2} \mathrm{O}$ or $6 \mathrm{UO}_{3} \cdot 5 \mathrm{CO}_{2} \cdot 7 \mathrm{H}_{2} \mathrm{O}$

Studtite Hydrated carbonate of $\mathrm{U}$ and $\mathrm{Pb}$.

Swartzite $\mathrm{CaMg}\left(\mathrm{UO}_{2}\right)\left(\mathrm{CO}_{3}\right)_{3} \cdot 12 \mathrm{H}_{2} \mathrm{O}$

Thorbastnaesite $\mathrm{Th}(\mathrm{Ce}, \mathrm{Ca})\left(\mathrm{CO}_{3}\right)_{2} \mathrm{~F}_{2} \cdot 3 \mathrm{H}_{2} \mathrm{O}$

Voglite $\mathrm{Ca}_{2} \mathrm{Cu}\left(\mathrm{UO}_{2}\right)\left(\mathrm{CO}_{3}\right)_{4} \cdot 6 \mathrm{H}_{2} \mathrm{O}$

Widenmannite Carbonate of $\mathrm{U}$ and $\mathrm{Pb}$.

Wyartite $\mathrm{UO}_{2} \cdot 6 \mathrm{UO}_{3} \cdot 2 \mathrm{CO}_{2} \cdot 3 \mathrm{CaO} \cdot 12-14 \mathrm{H}_{2} \mathrm{O}$

Zellerite $\mathrm{Ca}\left(\mathrm{UO}_{2}\right)\left(\mathrm{CO}_{3}\right)_{2} \cdot 5 \mathrm{H}_{2} \mathrm{O}$

See also Unnamed minerals, p. 42.

Molybdates:

Calcurmolite $\mathrm{Ca}\left(\mathrm{UO}_{2}\right)_{3}\left(\mathrm{MoO}_{4}\right)_{3}(\mathrm{OH})_{2} \cdot 11 \mathrm{H}_{2} \mathrm{O}$

Cousinite $\mathrm{MgO} \cdot 2 \mathrm{MoO}_{3} \cdot 2 \mathrm{UO}_{2} \cdot 6 \mathrm{H}_{2} \mathrm{O}$ 
Molybdates-Continued

Iriginite $\mathrm{UO}_{3} \cdot 2 \mathrm{MoO}_{3} \cdot 3 \mathrm{H}_{2} \mathrm{O}$

Moluranite $\mathrm{UO}_{2} \cdot 3 \mathrm{UO}_{3} \cdot 7 \mathrm{MoO}_{3} \cdot 20 \mathrm{H}_{2} \mathrm{O}$

Mourite Hydrous uranous uranic molybdate.

Sedovite $\mathrm{U}\left(\mathrm{MoO}_{4}\right)_{2}(?)$

Umohoite $\left(\mathrm{UO}_{2}\right)\left(\mathrm{MoO}_{4}\right) \cdot 4 \mathrm{H}_{2} \mathrm{O}(?)$

Wulfenite $\mathrm{Pb}(\mathrm{Mo}, \mathrm{U}) \mathrm{O}_{4}$

Niobates-tantalates-titanates:

Aeschynite $\left(\mathrm{Ce}, \mathrm{Ca}, \mathrm{Fe}^{+2}, \mathrm{Th}\right)(\mathrm{Ti}, \mathrm{Nb})_{2}(\mathrm{O}, \mathrm{OH})_{6}$

Betafite $A_{2-x} B_{2} \mathrm{O}_{6}\left(\mathrm{O}, \mathrm{OH}_{x}\right)$, where $A=\mathrm{Ca}, \mathrm{Na}, \mathrm{U}, \mathrm{Ce} ; B=\mathrm{Nb}, \mathrm{Ta}, \mathrm{Ti}$, Fe.

Brannerite $A B_{2} \mathrm{O}_{6}$, where $A=\mathrm{U}, \mathrm{Ca}, \mathrm{Fe}, \mathrm{Th}, \mathrm{Y} ; B=\mathrm{Ti}$ mainly and $\mathrm{Fe}$.

Calciosamarskite Probably (Ca, Y,Ce, U, Th $)_{3}(\mathrm{Nb}, \mathrm{Ta}, \mathrm{Fe}, \mathrm{Ti}, \mathrm{Sn})_{5} \mathrm{O}_{15}($ ?)

Davidite $A_{6} B_{15}(\mathrm{O}, \mathrm{OH})_{38}$, where $A=\mathrm{Fe}^{+2}$, rare earths, $\mathrm{U}^{+6}, \mathrm{Ca}, \mathrm{Zr}$, $\mathrm{Th}$; $B=\mathrm{Ti}, \mathrm{Fe}^{+3}$.

Euxenite ( $\mathrm{Y}, \mathrm{Ca}, \mathrm{Ce}, \mathrm{U}, \mathrm{Th})(\mathrm{Nb}, \mathrm{Ta}, \mathrm{Ti})_{2} \mathrm{O}_{6}$

Fergusonite (Y,Er,Ce,Fe) $(\mathrm{Nb}, \mathrm{Ta}, \mathrm{Ti}) \mathrm{O}_{4}$

Formanite $(\mathrm{Y}, \mathrm{U}, \mathrm{Th}, \mathrm{Ca})(\mathrm{Ta}, \mathrm{Nb}, \mathrm{Ti}) \mathrm{O}_{4}$

Hielmite $(\mathrm{Y}, \mathrm{Fe}, \mathrm{U})(\mathrm{Nb}, \mathrm{Ta}, \mathrm{Sn}, \mathrm{W})_{2} \mathrm{O}_{6}$

Ishikawaite (U,Fe, $\mathrm{Y}, \mathrm{Ce})(\mathrm{Nb}, \mathrm{Ta}) \mathrm{O}_{4}$

Khlopinite $(\mathrm{Y}, \mathrm{U}, \mathrm{Th})_{3}(\mathrm{Nb}, \mathrm{Ta}, \mathrm{Ti}, \mathrm{Fe})_{7} \mathrm{O}_{20}(?)$

Kobeite $A B_{2}(\mathrm{O}, \mathrm{OH})_{6}$, where $A=\mathrm{Y}, \mathrm{U} ; B=\mathrm{Ti}$ mainly, $\mathrm{Fe}^{+3}, \mathrm{Nb}$, Ta

Loparite $(\mathrm{Na}, \mathrm{Ce}, \mathrm{Th})_{1-x}(\mathrm{Nb}, \mathrm{Ti})\left[\mathrm{O}_{3-x}(\mathrm{OH})_{x}\right]$

Microlite $A_{2} B_{2} \mathrm{O}_{6}(\mathrm{O}, \mathrm{OH}, \mathrm{F})$, where $A=\mathrm{Na}, \mathrm{Ca}, \mathrm{Fe}^{+2}, \mathrm{U}^{+4}, \mathrm{Sb}^{+3}, \mathrm{~Pb}$, $\mathrm{Th}, \mathrm{Zr}, \mathrm{Ce}, \mathrm{Y} ; B=\mathrm{Nb}, \mathrm{Ta}, \mathrm{Ti}, \mathrm{Sn}, \mathrm{Fe}^{+3}$.

Niobo-aeschynite (Ce, $\mathrm{Y}, \mathrm{Ca}, \mathrm{Fe}, \mathrm{Th})(\mathrm{Nb}, \mathrm{Ti})_{2} \mathrm{O}_{6}$

Nohlite $\left(\mathrm{Ca}, \mathrm{Mg}, \mathrm{Fe}^{+2}, \mathrm{Y}, \mathrm{U}\right)_{2}\left(\mathrm{Nb}, \mathrm{Zr}, \mathrm{Fe}^{+3}\right)_{3} \mathrm{O}_{10}$

Obruchevite (Y, Na, Ca, U) (Nb, Ta, Ti, Fe) $)_{2}(\mathrm{O}, \mathrm{OH})_{7} \cdot \mathrm{H}_{2} \mathrm{O}$

Pisekite Niobate-tantalate-titanate of $\mathrm{U}$ and rare earths, with $\mathrm{Th}$ and Sn.

Polycrase $\quad(\mathrm{Y}, \mathrm{Ca}, \mathrm{Ce}, \mathrm{U}, \mathrm{Th})(\mathrm{Ti}, \mathrm{Nb}, \mathrm{Ta})_{2} \mathrm{O}_{6}$

Priorite ( $\left.\mathrm{Y}, \mathrm{Er}, \mathrm{Ca}, \mathrm{Fe}^{+2}, \mathrm{Th}\right)(\mathrm{Ti}, \mathrm{Nb})_{2} \mathrm{O}_{6}$

Pyrochlore $A_{2} B_{2} \mathrm{O}_{6}(\mathrm{O}, \mathrm{OH}, \mathrm{F})$, where $A=\mathrm{Na}, \mathrm{Ca}, \mathrm{U}, \mathrm{Th}, \mathrm{Ce}, \mathrm{Y} ; B=\mathrm{Nb}$, Ta, Ti.

Samarskite (Y, Ce, U, Ca, Fe, $\mathrm{Pb}, \mathrm{Th})(\mathrm{Nb}, \mathrm{Ta}, \mathrm{Ti}, \mathrm{Sn})_{2} \mathrm{O}_{6}$

Sinicite $A B_{2}(\mathrm{O}, \mathrm{OH})_{7}$, where $A=\mathrm{Ce}, \mathrm{Y}, \mathrm{Th}, \mathrm{U} ; B=\mathrm{Ti}$, Nb mainly

Thorutite ( $\mathrm{Th}, \mathrm{Ca}, \mathrm{U}) \mathrm{Ti}_{2}(\mathrm{O}, \mathrm{OH})_{6}$

Yttrocrasite (Y,Th, U, Ca) ${ }_{2} \mathrm{Ti}_{4} \mathrm{O}_{11}(?)$

Yttrotantalite $(\mathrm{Fe}, \mathrm{Y}, \mathrm{U})(\mathrm{Nb}, \mathrm{Ta}) \mathrm{O}_{4}$

Zirkelite (Ca,Fe,Th, $\mathrm{U})_{2}(\mathrm{Ti}, \mathrm{Nb}, \mathrm{Zr})_{2} \mathrm{O}_{7}(?)$

Oxides:

Becquerelite $7 \mathrm{UO}_{3} \cdot 11 \mathrm{H}_{2} \mathrm{O}$

Billietite $\mathrm{BaO} \cdot 6 \mathrm{UO}_{3} \cdot 11 \mathrm{H}_{2} \mathrm{O}$

Cerianite $(\mathrm{Ce}, \mathrm{Th}) \mathrm{O}_{2}$

Clarkeite (Na, $\left.\mathrm{Ca}, \mathrm{Pb}, \mathrm{Th}, \mathrm{H}_{2} \mathrm{O}\right) \mathrm{U}_{2}\left(\mathrm{O}, \mathrm{H}_{2} \mathrm{O}\right)_{7}$

Compreignacite $\mathrm{K}_{2} \mathrm{O} \cdot 6 \mathrm{UO}_{3} \cdot 11 \mathrm{H}_{2} \mathrm{O}$

Curite $3 \mathrm{PbO} \cdot 8 \mathrm{UO}_{3} \cdot 5 \mathrm{H}_{2} \mathrm{O}$

Fourmarierite $\mathrm{PbO} \cdot 4 \mathrm{UO}_{3} \cdot 5 \mathrm{H}_{2} \mathrm{O}$

Hydronasturan $\mathrm{UO}_{2} \cdot k \mathrm{UO}_{3} \cdot n \mathrm{H}_{2} \mathrm{O}$, where $k=2.3$ to $5.0 ; n=3.9$ to 9.0

Ianthininite $\mathrm{UO}_{2} \cdot 5 \mathrm{UO}_{3} \cdot 10-11 \mathrm{H}_{2} \mathrm{O}$

Masuyite $\mathrm{UO}_{3} \cdot 2 \mathrm{H}_{2} \mathrm{O}$

Parapitchblende Oxide of uranium, chiefly $\mathrm{U}^{+6}$, minor $\mathrm{U}^{+4}$. 
Oxides-Continued

Richetite Contains $\mathrm{Pb}$ and $\mathrm{U}$.

Schoepite $\mathrm{UO}_{3} \cdot 2 \mathrm{H}_{2} \mathrm{O}$

Thorianite $(\mathrm{Th}, \mathrm{U}) \mathrm{O}_{2}$

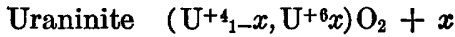

Uranosphaerite $\mathrm{BiUO}_{4}(\mathrm{OH})$

Urgite $\mathrm{UO}_{3} \cdot n \mathrm{H}_{2} \mathrm{O}$

Vandenbrandeite $\mathrm{CuUO}_{4} \cdot 2 \mathrm{H}_{2} \mathrm{O}$

Vandendriesscheite $\mathrm{PbO} \cdot 7 \mathrm{UO}_{3} \cdot 12 \mathrm{H}_{2} \mathrm{O}$ (?)

Wölsendorfite $(\mathrm{Pb}, \mathrm{Ca}) \mathrm{U}_{2} \mathrm{O}_{7} \cdot 2 \mathrm{H}_{2} \mathrm{O}$

See also Unnamed minerals, p. 43.

Phosphates:

Autunite $\mathrm{Ca}\left(\mathrm{UO}_{2}\right)_{2}\left(\mathrm{PO}_{4}\right)_{2} \cdot 10-12 \mathrm{H}_{2} \mathrm{O}$

Bassetite $\mathrm{Fe}\left(\mathrm{UO}_{2}\right)_{2}\left(\mathrm{PO}_{4}\right)_{2} \cdot 8 \mathrm{H}_{2} \mathrm{O}$

Bergenite $\mathrm{Ba}\left(\mathrm{UO}_{2}\right)_{4}\left(\mathrm{PO}_{4}\right)_{2}(\mathrm{OH})_{4} \cdot 8 \mathrm{H}_{2} \mathrm{O}$

Brockite (Ca,Th) $\mathrm{PO}_{4} \cdot \mathrm{H}_{2} \mathrm{O}$

Cerphosphorhuttonite (Th, Ce) $(\mathrm{Si}, \mathrm{P}) \mathrm{O}_{4} \cdot 1.5 \mathrm{H}_{2} \mathrm{O}$

Cheralite $(\mathrm{Ca}, \mathrm{Th}, \mathrm{Ce})(\mathrm{P}, \mathrm{Si}) \mathrm{O}_{4}$

Coconinoite $\mathrm{Fe}^{+3}{ }_{2} \mathrm{Al}_{2}\left(\mathrm{UO}_{2}\right)_{2}\left(\mathrm{PO}_{4}\right)_{4}\left(\mathrm{SO}_{4}\right)(\mathrm{OH})_{2} \cdot 20 \mathrm{H}_{2} \mathrm{O}$

Dewindtite $\mathrm{Pb}_{3}\left(\mathrm{UO}_{2}\right)_{5}\left(\mathrm{PO}_{4}\right)_{4}(\mathrm{OH})_{4} \cdot 10 \mathrm{H}_{2} \mathrm{O}(?)$

Dumontite $\mathrm{Pb}_{2}\left(\mathrm{UO}_{2}\right)_{3}\left(\mathrm{PO}_{4}\right)_{2}(\mathrm{OH})_{4} \cdot 3 \mathrm{H}_{2} \mathrm{O}$

Fritzscheite $\mathrm{Mn}\left(\mathrm{UO}_{2}\right)_{2}\left[(\mathrm{P}, \mathrm{V}) \mathrm{O}_{4} \mathrm{l}_{2} \cdot 8 \mathrm{H}_{2} \mathrm{O}(?)\right.$

Grayite Thorium phosphate, perhaps $(\mathrm{Th}, \mathrm{Pb}, \mathrm{Ca})\left(\mathrm{PO}_{4}\right) \cdot \mathrm{H}_{2} \mathrm{O}$

Hydrogen autunite $\mathrm{H}_{2}\left(\mathrm{UO}_{2}\right)_{2}\left(\mathrm{PO}_{4}\right)_{2} \cdot 8-10 \mathrm{H}_{2} \mathrm{O}$

Kivuite (Th, $\mathrm{Ca}, \mathrm{Pb}) \mathrm{H}_{2}\left(\mathrm{UO}_{2}\right)_{4}\left(\mathrm{PO}_{4}\right)_{2}(\mathrm{OH})_{8} \cdot 7 \mathrm{H}_{2} \mathrm{O}$

Lermontovite (U,Ca, R.E. $\left.{ }^{*}\right)_{3}\left(\mathrm{PO}_{4}\right)_{4} \cdot 6 \mathrm{H}_{2} \mathrm{O}$

Meta-ankoleite $\mathrm{K}_{2}\left(\mathrm{UO}_{2}\right)_{2}\left(\mathrm{PO}_{4}\right)_{2} \cdot 6 \mathrm{H}_{2} \mathrm{O}$

Meta-autunite I $\mathrm{Ca}\left(\mathrm{UO}_{2}\right)_{2}\left(\mathrm{PO}_{4}\right)_{2} \cdot 2-6 \mathrm{H}_{2} \mathrm{O}$

Meta-autunite II $\mathrm{Ca}\left(\mathrm{UO}_{2}\right)_{2}\left(\mathrm{PO}_{4}\right)_{2} \cdot 0-6 \mathrm{H}_{2} \mathrm{O}$

Metasaléeite $\mathrm{Mg}\left(\mathrm{UO}_{2}\right)_{2}\left(\mathrm{PO}_{4}\right)_{2} \cdot 4(?) \mathrm{H}_{2} \mathrm{O}$

Metatorbernite $\mathrm{Cu}\left(\mathrm{UO}_{2}\right)_{2}\left(\mathrm{PO}_{4}\right)_{2} \cdot n \mathrm{H}_{2} \mathrm{O}$, where $n=4(?)$ to 8 .

Meta-uranocircite $\mathrm{Ba}\left(\mathrm{UO}_{2}\right)_{2}\left(\mathrm{PO}_{4}\right)_{2} \cdot 8 \mathrm{H}_{2} \mathrm{O}$

Monazite (Ce,La, $\mathrm{Nd}) \mathrm{PO}_{4}$, with Th substituting for (Ce,La).

Natroautunite $\mathrm{Na}_{2}\left(\mathrm{UO}_{2}\right)_{2}\left(\mathrm{PO}_{4}\right)_{2} \cdot 8 \mathrm{H}_{2} \mathrm{O}$

Ningyoite (U,Ca, R.E.* $)_{2}\left(\mathrm{PO}_{4}\right)_{2} \cdot 1-2 \mathrm{H}_{2} \mathrm{O}$

Parsonsite $\left.\mathrm{Pb}_{2}\left(\mathrm{UO}_{2}\right) \mathrm{PO}_{4}\right)_{2}$

Phosphuranylite $\mathrm{Ca}\left(\mathrm{UO}_{2}\right)_{4}\left(\mathrm{PO}_{4}\right)_{2}(\mathrm{OH})_{4} \cdot 7 \mathrm{H}_{2} \mathrm{O}$

Przhevalskite $\mathrm{Pb}\left(\mathrm{UO}_{2}\right)_{2}\left(\mathrm{PO}_{4}\right)_{2} \cdot 2 \mathrm{H}_{2} \mathrm{O}$

Pseudo-autunite $\left(\mathrm{H}_{3} \mathrm{O}\right)_{2} \mathrm{Ca}\left(\mathrm{UO}_{2}\right)\left(\mathrm{PO}_{4}\right)_{2} \cdot 2.5 \mathrm{H}_{2} \mathrm{O}$

Renardite $\mathrm{Pb}\left(\mathrm{UO}_{2}\right)_{4}\left(\mathrm{PO}_{4}\right)_{2}(\mathrm{OH})_{4} \cdot 7 \mathrm{H}_{2} \mathrm{O}$

Sabugalite $\mathrm{HAl}\left(\mathrm{UO}_{2}\right)_{4}\left(\mathrm{PO}_{4}\right)_{4} \cdot 16 \mathrm{H}_{2} \mathrm{O}$

Saléeite $\mathrm{Mg}\left(\mathrm{UO}_{2}\right)_{2}\left(\mathrm{PO}_{4}\right)_{2} \cdot 8-10 \mathrm{H}_{2} \mathrm{O}$

Saryarkite $(\mathrm{Ca}, \mathrm{Y}, \mathrm{Th})_{2} \mathrm{Al}_{4}\left(\mathrm{SiO}_{4}, \mathrm{PO}_{4}\right)_{4}(\mathrm{OH}) \cdot 9 \mathrm{H}_{2} \mathrm{O}$

Sodium uranospinite $\left(\mathrm{Na}_{2}, \mathrm{Ca}\right)\left(\mathrm{UO}_{2}\right)_{2}\left[(\mathrm{As}, \mathrm{P}) \mathrm{O}_{4}\right]_{2} \cdot 5 \mathrm{H}_{2} \mathrm{O}$

Torbernite $\mathrm{Cu}\left(\mathrm{UO}_{2}\right)_{2}\left(\mathrm{PO}_{4}\right)_{2} \cdot 12 \mathrm{H}_{2} \mathrm{O}$

Uramphite $\left(\mathrm{NH}_{4}\right)\left(\mathrm{UO}_{2}\right)\left(\mathrm{PO}_{4}\right) \cdot 3 \mathrm{H}_{2} \mathrm{O}$

Uranospathite $\mathrm{Cu}\left(\mathrm{UO}_{2}\right)_{2}\left(\mathrm{AsO}_{4}, \mathrm{PO}_{4}\right)_{2} \cdot 11 \mathrm{H}_{2} \mathrm{O}($ ?)

See also. Unnamed minerals, p. 42-43.

Selenites:

Demesmaekerite $\mathrm{Pb}_{2} \mathrm{Cu}_{5}\left(\mathrm{UO}_{2}\right)_{2}\left(\mathrm{SeO}_{3}\right)_{6}(\mathrm{OH})_{6} \cdot 2 \mathrm{H}_{2} \mathrm{O}$

Guilleminite $\mathrm{Ba}\left(\mathrm{UO}_{2}\right)_{3}(\mathrm{OH})_{4}\left(\mathrm{SeO}_{3}\right)_{2} \cdot 3 \mathrm{H}_{2} \mathrm{O}$

- Rare earths. 
Silicates:

Barium uranophane Contains major $\mathrm{Ba}, \mathrm{U}, \mathrm{Si}$.

Beta-uranophane $\mathrm{Ca}\left(\mathrm{UO}_{2}\right)_{2}\left(\mathrm{SiO}_{3}\right)_{2}(\mathrm{OH})_{2} \cdot 5 \mathrm{H}_{2} \mathrm{O}$

Bilibinite $3(\mathrm{Ca}, \mathrm{Pb}) \mathrm{O} \cdot(\mathrm{U}, \mathrm{Th}) \mathrm{O}_{2} \cdot 7 \mathrm{UO}_{3} \cdot 10 \mathrm{SiO}_{2} \cdot 19 \mathrm{H}_{2} \mathrm{O}$

Boltwoodite $\mathrm{K}_{2}\left(\mathrm{UO}_{2}\right)_{2}\left(\mathrm{SiO}_{3}\right)_{2}(\mathrm{OH})_{2} \cdot 5 \mathrm{H}_{2} \mathrm{O}$

Caryocerite Borosilicate of $\mathrm{Ce}, \mathrm{Y}$, and Th.

Cerphosphorhuttonite (Th, Ce) $(\mathrm{Si}, \mathrm{P}) \mathrm{O}_{4} \cdot 1.5 \mathrm{H}_{2} \mathrm{O}$

Cheralite $(\mathrm{Ca}, \mathrm{Th}, \mathrm{Ce})(\mathrm{P}, \mathrm{Si}) \mathrm{O}_{4}$

Chevkinite (Ce, Y, Ca, U,Th) ${ }_{2}(\mathrm{Ti}, \mathrm{Fe}, \mathrm{Mg})_{2}(\mathrm{Si}, \mathrm{Al})_{2} \mathrm{O}_{11}(?)$

Coffinite $\mathrm{U}\left(\mathrm{SiO}_{4}\right)_{1-x}(\mathrm{OH})_{4 x}$

Cuprosklodowskite $\mathrm{Cu}\left(\mathrm{UO}_{2}\right)_{2}\left(\mathrm{SiO}_{3}\right)_{2}(\mathrm{OH})_{2} \cdot 5 \mathrm{H}_{2} \mathrm{O}$

Ekanite $(\mathrm{Th}, \mathrm{U})(\mathrm{Ca}, \mathrm{Fe}, \mathrm{Pb})_{2} \mathrm{Si}_{8} \mathrm{O}_{20}$

Haiweeite $\mathrm{CaO} \cdot 2 \mathrm{UO}_{3} \cdot 6 \mathrm{SiO}_{2} \cdot 5 \mathrm{H}_{2} \mathrm{O}$

Huttonite $\mathrm{ThSiO}_{4}$

Hydrocerite $(\mathrm{La}, \mathrm{Ce}, \mathrm{Th})_{2}(\mathrm{Si}, \mathrm{P})_{2} \mathrm{O}_{7} \cdot 5 \mathrm{H}_{2} \mathrm{O}$

Kasolite $\mathrm{Pb}\left(\mathrm{UO}_{2}\right)\left(\mathrm{SiO}_{3}\right)(\mathrm{OH})_{2}$

Metahaiweeite $\mathrm{CaO} \cdot 2 \mathrm{UO}_{3} \cdot 6 \mathrm{SiO}_{2} \cdot n \mathrm{H}_{2} \mathrm{O}$

Nenadkevite $\left(\mathrm{U}^{+4}, \mathrm{Y}, \mathrm{Ce}\right) \mathrm{U}^{+8}(\mathrm{Ca}, \mathrm{Mg}, \mathrm{Pb})\left(\mathrm{SiO}_{4}\right)_{2}(\mathrm{OH})_{4} \cdot n \mathrm{H}_{2} \mathrm{O}$

Orlite $3 \mathrm{PbO} \cdot 3 \mathrm{UO}_{3} \cdot 4 \mathrm{SiO}_{2} \cdot 6 \mathrm{H}_{2} \mathrm{O}$

Orthochevkinite Dimorph of chevkinite.

Perrierite (Ce, $\mathrm{Y}, \mathrm{Ca}, \mathrm{Th})_{2}(\mathrm{Ti}, \mathrm{Fe}, \mathrm{Mg})_{2}(\mathrm{Si}, \mathrm{Al})_{2} \mathrm{O}_{11}(?)$

Ranquilite $3 \mathrm{CaO} \cdot 4 \mathrm{UO}_{3} \cdot 10 \mathrm{SiO}_{2} \cdot 24 \mathrm{H}_{2} \mathrm{O}$

Saryarkite $(\mathrm{Ca}, \mathrm{Y}, \mathrm{Th})_{2} \mathrm{Al}_{4}\left(\mathrm{SiO}_{4}, \mathrm{PO}_{4}\right)_{4}(\mathrm{OH}) \cdot 9 \mathrm{H}_{2} \mathrm{O}$

Sklodowskite $\mathrm{Mg}\left(\mathrm{UO}_{2}\right)_{2}\left(\mathrm{SiO}_{3}\right)_{2}(\mathrm{OH})_{2} \cdot 5 \mathrm{H}_{2} \mathrm{O}$

Soddyite $\left(\mathrm{UO}_{2}\right)_{5}\left(\mathrm{SiO}_{4}\right)_{2}(\mathrm{OH})_{2} \cdot 5 \mathrm{H}_{2} \mathrm{O}$

Steenstrupine $(\mathrm{Ce}, \mathrm{La}, \mathrm{Th}, \mathrm{Ca}, \mathrm{Na})_{2}(\mathrm{Mn}, \mathrm{Fe})\left(\mathrm{SiO}_{3}\right)_{2}(\mathrm{OH})_{2} \cdot 2 \mathrm{H}_{2} \mathrm{O}$

Thorite $\mathrm{ThSiO}_{4}$

Thorogummite $\mathrm{Th}\left(\mathrm{SiO}_{4}\right)_{1-x}(\mathrm{OH})_{4 x}$

Thorosteenstrupine $(\mathrm{Ca}, \mathrm{Th}, \mathrm{Mn})_{3} \mathrm{Si}_{4}(\mathrm{O}, \mathrm{F})_{12} \cdot 5.7 \mathrm{H}_{2} \mathrm{O}$

Tritomite Borosilicate of $\mathrm{Ce}, \mathrm{Y}, \mathrm{Th}, \mathrm{Ca}$, and $\mathrm{F}$.

Uranophane $\mathrm{Ca}\left(\mathrm{UO}_{2}\right)_{2}\left(\mathrm{SiO}_{3}\right)_{2}(\mathrm{OH})_{2} \cdot 5 \mathrm{H}_{2} \mathrm{O}$

Ursilite $2(\mathrm{Ca}, \mathrm{Mg}) \mathrm{O} \cdot 2 \mathrm{UO}_{3} \cdot 5 \mathrm{SiO}_{2} \cdot 9-10 \mathrm{H}_{2} \mathrm{O}$

Usigite $R \uparrow\left(\mathrm{UO}_{2}\right)_{2}\left(\mathrm{Si}_{2} \mathrm{O}_{7}\right) \cdot n \mathrm{H}_{2} \mathrm{O}$

Weeksite $\mathrm{K}\left(\mathrm{UO}_{2}\right)_{2}\left(\mathrm{Si}_{2} \mathrm{O}_{5}\right)_{3} \cdot 4 \mathrm{H}_{2} \mathrm{O}$

Yttrialite $(\mathrm{Y}, \mathrm{Th})_{2} \mathrm{Si}_{2} \mathrm{O}_{7}$

See also Unnamed minerals, p. 43.

Sulfates:

Calciouraconite $\mathrm{Near} \mathrm{Ca}\left(\mathrm{UO}_{2}\right)_{4}\left(\mathrm{SO}_{4}\right)_{2}(\mathrm{OH})_{6} \cdot 20 \mathrm{H}_{2} \mathrm{O}$

Coconinoite $\mathrm{Fe}_{2}{ }^{+3} \mathrm{Al}_{2}\left(\mathrm{UO}_{2}\right)_{2}\left(\mathrm{PO}_{4}\right)_{4}\left(\mathrm{SO}_{4}\right)(\mathrm{OH})_{2} \cdot 20 \mathrm{H}_{2} \mathrm{O}$

Cuprozippeite $\mathrm{Cu}\left(\mathrm{UO}_{2}\right)_{2}\left(\mathrm{SO}_{4}\right)_{2}(\mathrm{OH})_{2} \cdot 11 \mathrm{H}_{2} \mathrm{O}$

Johannite $\mathrm{Cu}\left(\mathrm{UO}_{2}\right)_{2}\left(\mathrm{SO}_{4}\right)_{2}(\mathrm{OH})_{2} \cdot 6 \mathrm{H}_{2} \mathrm{O}$

Medjidite Supposedly a uranium sulfate.

Meta-uranopilite $\left(\mathrm{UO}_{2}\right)_{6}\left(\mathrm{SO}_{4}\right)(\mathrm{OH})_{10} \cdot 5 \mathrm{H}_{2} \mathrm{O}($ ?)

Schroeckingerite $\mathrm{NaCa}_{3}\left(\mathrm{UO}_{2}\right)\left(\mathrm{CO}_{3}\right)_{3}\left(\mathrm{SO}_{4}\right) \mathrm{F} \cdot 10 \mathrm{H}_{2} \mathrm{O}$

Uranochalcite Supposedly a uranium sulfate.

Uranopilite $\left(\mathrm{UO}_{2}\right)_{6}\left(\mathrm{SO}_{4}\right)(\mathrm{OH})_{10} \cdot 12 \mathrm{H}_{2} \mathrm{O}$

Voglianite Hydrous calcium uranium sulfate.

Zippeite $\mathrm{K}_{4}\left(\mathrm{UO}_{2}\right)_{6}\left(\mathrm{SO}_{4}\right)_{3}(\mathrm{OH})_{10} \cdot \mathrm{H}_{2} \mathrm{O}$

Tellurites:

Moctezumite $\mathrm{PbO} \cdot \mathrm{UO}_{3} \cdot 2 \mathrm{TeO}_{2}$

$\dagger$ Meaning of $R$ not specified. 
Vanadates:

Carnotite $\mathrm{K}_{2}\left(\mathrm{UO}_{2}\right)_{2}\left(\mathrm{VO}_{4}\right)_{2} \cdot n \mathrm{H}_{2} \mathrm{O}(n=1$ to 3$)$

Ferghanite $\left(\mathrm{UO}_{2}\right)_{3}\left(\mathrm{VO}_{4}\right)_{2} \cdot 6 \mathrm{H}_{2} \mathrm{O}($ ? $)$

Francevillite $(\mathrm{Ba}, \mathrm{Pb})\left(\mathrm{UO}_{2}\right)_{2}\left(\mathrm{VO}_{4}\right)_{2} \cdot 5 \mathrm{H}_{2} \mathrm{O}$

Fritzscheite $\mathrm{Mn}\left(\mathrm{UO}_{2}\right)_{2}\left[(\mathrm{P}, \mathrm{V}) \mathrm{O}_{4}\right]_{2} \cdot 8 \mathrm{H}_{2} \mathrm{O}(?)$

Metatyuyamunite $\mathrm{Ca}\left(\mathrm{UO}_{2}\right)_{2}\left(\mathrm{VO}_{4}\right)_{2} \cdot 3-5 \mathrm{H}_{2} \mathrm{O}$

Rauvite $\mathrm{CaO} \cdot 2 \mathrm{UO}_{3} \cdot 5 \mathrm{~V}_{2} \mathrm{O}_{5} \cdot 16 \mathrm{H}_{2} \mathrm{O}(?)$

Sengierite $\mathrm{Cu}\left(\mathrm{UO}_{2}\right)_{2}\left(\mathrm{VO}_{4}\right)_{2} \cdot 8-10 \mathrm{H}_{2} \mathrm{O}$

Tyuyamunite $\mathrm{Ca}\left(\mathrm{UO}_{2}\right)_{2}\left(\mathrm{VO}_{4}\right)_{2} \cdot 5-8 \mathrm{H}_{2} \mathrm{O}$

Uvanite $\mathrm{U}_{2} \mathrm{~V}_{6} \mathrm{O}_{21} \cdot 15 \mathrm{H}_{2} \mathrm{O}($ ?)

Vanuralite $\left(\mathrm{UO}_{2}\right)_{2} \mathrm{Al}\left(\mathrm{VO}_{4}\right)_{2}(\mathrm{OH}) \cdot 8 \mathrm{H}_{2} \mathrm{O}$

Vanuranylite $\left[\left(\mathrm{H}_{3} \mathrm{O}\right)_{1.2}(\mathrm{Ba}, \mathrm{Ca}, \mathrm{K}, \mathrm{Pb})_{0.4}\right]\left(\mathrm{UO}_{2}\right)_{2}\left(\mathrm{VO}_{4}\right)_{2} \cdot 4.2 \mathrm{H}_{2} \mathrm{O}$

See also Unnamed minerals, p. 43. 


\section{A. URANIUM AND THORIUM MINERALS}

\section{ABERNATHYITE}

$\mathrm{K}_{2}\left(\mathrm{UO}_{2}\right)_{2}\left(\mathrm{AsO}_{4}\right)_{2} \cdot 6 \mathrm{H}_{2} \mathrm{O}$

Potassium member of the meta-autunite group.

$\mathrm{U}=45.8$ percent.

Color: Yellow. A rare secondary mineral.

Am. Mineralogist, v. 41, p. 82-90 (1956).

AESCHYNITE (or eschynite)

$\left(\mathrm{Ce}, \mathrm{Ca}, \mathrm{Fe}^{+2}, \mathrm{Th}\right)(\mathrm{Ti}, \mathrm{Nb})_{2}(\mathrm{O}, \mathrm{OH})_{6}$

Part of the aeschynite-priorite series.

$\mathrm{Th}=9.9$ to 25.8 percent, $\mathrm{U}=$ up to 2.5 percent.

Color: Black to brown. From granite pegmatites and nepheline syenites.

Dana VII, v. 1, p. 793-796.

Mineralog. Mag., v. 31, p. 763-780 (1958).

Alumo-aeschynite

Aluminian aeschynite.

$\mathrm{Th}=1.6$ percent.

Am. Mineralogist, v. 50, p. 2101-2102 (1965).

Lyndochite

Variety of aeschynite, relatively high in $\mathrm{Ca}$ and $\mathrm{Th}$ and low in $\mathrm{U}$.

Dana VII, v. 1, p. 787-791.

Mineralog. Mag., v. 35, p. 801-809 (1966).

See also Niobo-aeschynite, p. 56.

Thoro-aeschynite

A thorium-rich variety.

$\mathrm{Th}=25.8$ percent, $\mathrm{U}=\mathbf{0 . 8}$ percent.

From microcline veinlets in the contact zone of biotite syenites and gneisses.

Am. Mineralogist, v. 50, p. 2101 (1965).

\section{ANDERSONITE}

$\mathrm{Na}_{2} \mathrm{Ca}\left(\mathrm{UO}_{2}\right)\left(\mathrm{CO}_{3}\right)_{3} \cdot 6 \mathrm{H}_{2} \mathrm{O}$

$\mathrm{U}=39.2$ percent.

Color: Bright green. Rare secondary mineral.

Dana VII, v. 2, p. 239.

\section{ARSENURANYLITE}

$\mathrm{Ca}\left(\mathrm{UO}_{2}\right)_{4}\left(\mathrm{AsO}_{4}\right)_{2}(\mathrm{OH})_{4} \cdot 6 \mathrm{H}_{2} \mathrm{O}$

Arsenic analog of phosphuranylite.

$\mathrm{U}=\mathbf{5 8 . 3}$ percent.

Color: Orange yellow. A secondary mineral.

Am. Mineralogist, v. 44, p. 208 (1959). 


\section{AUTUNITE}

$\mathrm{Ca}\left(\mathrm{UO}_{2}\right)_{2}\left(\mathrm{PO}_{4}\right)_{2} \cdot 10-12 \mathrm{H}_{2} \mathrm{O}$

$\mathrm{U}=48.3$ to 50.1 percent.

Color: Yellow. Common secondary mineral.

Dana VII, v. 2, p. 984-987.

Soc. Française Minéralogie et Cristallographie Bull., v. 81, p. 4-10 (1958).

Calciumphosphoruranit

Synonym of autunite.

Mineralog. Mag., v. 28, p. 732 (1949).

Calcouranit

Synonym of autunite.

Berg- u. Hüttenm. Zeitung., v. 24, p. 302 (1865).

Chalco-uranite

Synonym of autunite.

Royal Soc. South Australia Trans., v. 68, p. 334-357 (1944).

Lime-uranite

Synonym of autunite.

Brooke, H. J., and Miller, W. H., Introduction to Mineralogy, p. 519 (1852).

See also Meta-autunite, p. 24.

\section{BARIUM URANOPHANE}

Contains $\mathrm{Ba}, \mathrm{U}, \mathrm{Si}$, with a little $\mathrm{Mo}, \mathrm{Al}$, and $\mathrm{Ca}$.

Needs further study.

Color: Yellow. A secondary mineral.

United Nations Internat. Conf. on Peaceful Uses of Atomic Energy, 2d., Geneva, Proc., v. 2, p. 295 (1958).

Am. Mineralogist, v. 44, p. 466-467 (1959).

\section{BASSETITE}

$\mathrm{Fe}\left(\mathrm{UO}_{2}\right)_{2}\left(\mathrm{PO}_{4}\right)_{2} \cdot 8 \mathrm{H}_{2} \mathrm{O}$

$\mathrm{U}=51.0$ percent.

Color: Yellow. Rare secondary mineral.

Dana VII, v. 2, p. 994-995.

Mineralog. Mag., v. 30, p. 343-353 (1954).

Iron uranite

Incompletely examined mineral from New Mexico.

May be bassetite or altered bassetite.

U.S. Geol. Survey Bull. 1064, p. 204 (1958).

\section{BAYLEYITE}

$\mathrm{Mg}_{2}\left(\mathrm{UO}_{2}\right)\left(\mathrm{CO}_{3}\right)_{3} \cdot 18 \mathrm{H}_{2} \mathrm{O}$

$\mathrm{U}=28.9$ percent.

Color: Yellow. Rare secondary mineral.

Dana VII, v. 2, p. 237-238.

\section{BECQUERELITE}

$7 \mathrm{UO}_{3} \cdot 11 \mathrm{H}_{2} \mathrm{O}$

Isostructural with billietite and both natural and synthetic $\mathrm{CaO} \cdot 6 \mathrm{UO}_{3} \cdot 11 \mathrm{H}_{2} \mathrm{O}$.

$\mathrm{U}=75.7$ percent.

Color: Amber to yellow. Uncommon secondary mineral. 
BECQUERELITE-Continued

Am. Mineralogist, v. 38, p. 1019-1024 (1953); v. 42, p. 920 (1957).

Acad. sci. [Paris] Comptes Rendus, v. 244, p. 91-93 (1959).

Soc. Française Minéralogie et Cristallographie Bull., v. 82, p. 246-249 (1959).

\section{BERGENITE}

$\mathrm{Ba}\left(\mathrm{UO}_{2}\right)_{4}\left(\mathrm{PO}_{4}\right)_{2}(\mathrm{OH})_{4} \cdot 8 \mathrm{H}_{2} \mathrm{O}$.

Barium analog of phosphuranylite.

$\mathrm{U}=58.8$ percent.

Color: Yellow. Rare secondary mineral.

Neues Jahrb. Mineralogie Monatsh. 1959, no. 10, p. 232-233.

Am. Mineralogist, v. 41, p. 918-920 (1956).

Barium-phosphuranylite

Synonym of bergenite.

\section{BETAFITE}

Essentially a uranium-rich pyrochlore.

$A_{2-x} B_{2} \mathrm{O}_{6}\left(\mathrm{O}, \mathrm{OH}_{x}\right)$, with $A=\mathrm{Ca}, \mathrm{Na}, \mathrm{U}, \mathrm{Ce}, \mathrm{Y} ; B=\mathrm{Nb}, \mathrm{Ta}, \mathrm{Ti}, \mathrm{Fe}^{+3}$.

It probably forms a continuous series with pyrochlore; the name betafite is arbitrarly assigned to members of the series with $U$ greater than 15 percent.

Color: Yellow, brown, black.

From granitic pegmatites, alkalic rocks, carbonatites.

$\mathrm{U}=15-24.5$ percent; $\mathrm{Th}=1.0-1.1$ percent.

U.S. Geol. Survey Bull. 1064, p. 320-325 (1958).

Canadian Mineralogist, v. 6, p. 610-633 (1961).

Aluminobetafite

Perhaps an aluminum-rich variety of betafite $\left(\mathrm{Al}_{2} \mathrm{O}_{3}=15.7\right.$ percent), but needs further study.

$\mathrm{U}=18.1$ percent, $\mathrm{Th}=$ less than 1.4 percent.

Am. Mineralogist, v. 48, p. 1183 (1963).

Blomstrandite

Synonym of betafite.

$\mathrm{U}=16.3$ percent.

Dana VII, v. 1, p. 803-804.

Mendeleyevite

A titanian betafite.

$\mathrm{U}=$ up to 20 percent.

Dana VII, v. 1, p. $803-804$.

Samiresite

Possibly a plumboan betafite, but perhaps an independent species.

$\mathrm{U}=$ up to 18.7 percent.

Dana VII, v. 1, p. 803-805.

Am. Mineralogist, v. 51, p. 1551 (1966).

BETA-URANOPHANE

$\mathrm{Ca}\left(\mathrm{UO}_{2}\right)_{2}\left(\mathrm{SiO}_{3}\right)_{2}(\mathrm{OH})_{2} \cdot 5 \mathrm{H}_{2} \mathrm{O}$

Dimorphous with uranophane.

$\mathrm{U}=55.6$ percent.

Color: Yellow. Secondary mineral.

Am. Mineralogist, v. 35, p. 245-250 (1950); v. 40, p. 634-635 (1955).

Soc. Française Minéralogie et Cristallographie Bull., v. 74, p. 457-488 (1951). 
BETA-URANOPHANE-Continued

Beta-uranotile

Synonym of beta-uranophane.

České Spol. Nauk Věstnǐk, v. 2, p. 25 (1935).

Randite

A mixture of beta-uranophane, some tyuyamunite, and calcite.

Am. Mineralogist, v. 35, p. 245-250 (1950).

BILIBINITE

$3(\mathrm{Ca}, \mathrm{Pb}) \mathrm{O} \cdot(\mathrm{U}, \mathrm{Th}) \mathrm{O}_{2} \cdot 7 \mathrm{UO}_{3} \cdot 10 \mathrm{SiO}_{2} \cdot 19 \mathrm{H}_{2} \mathrm{O}$

Extreme uranium member in a series of amorphous thorium-uranium silicates of variable composition.

$\mathrm{U}=42.7$ percent; $\mathrm{Th}=1.3$ percent.

Color: Black. Occurs as cementing material in sandstones.

Am. Mineralogist, v. 44, p. 692 (1959).

Compare Thorogummite, p. 39.

\section{BILLIETITE}

$\mathrm{BaO} \cdot 6 \mathrm{UO}_{3} \cdot 11 \mathrm{H}_{2} \mathrm{O}$ or $\mathrm{Ba}_{6} \mathrm{O}_{16}(\mathrm{OH})_{6} \cdot 8 \mathrm{H}_{2} \mathrm{O}$

Isostructural with becquerelite and natural and synthetic $\mathrm{CaO} \cdot 6 \mathrm{UO}_{3}$. $11 \mathrm{H}_{2} \mathrm{O}$.

Barium analog of compreignacite.

$\mathrm{U}=68.8$ percent.

Color: Yellow. A rare secondary mineral.

Am. Mineralogist, v. 38, p. 1019-1024 (1953).

Soc. Française Minéralogie et Cristallographie Bull., v. 82, p. 252-254 (1959).

\section{BOLTWOODITE}

$\mathrm{K}_{2}\left(\mathrm{UO}_{2}\right)_{2}\left(\mathrm{SiO}_{3}\right)_{2}(\mathrm{OH})_{2} \cdot 5 \mathrm{H}_{2} \mathrm{O}$

$\mathrm{U}=58.2$ percent.

Color: Yellow. Secondary mineral in sandstone.

Am. Mineralogist, v. 42, p. 307 (1957).

\section{BRANNERITE}

$A B_{2} \mathrm{O}_{6}$ with $A=\mathrm{U}$ mainly, also $\mathrm{Ca}, \mathrm{Fe}, \mathrm{Th}, \mathrm{Y} ; B=\mathrm{Ti}$ mainly and $\mathrm{Fe}$.

$\mathrm{U}=26.5$ to 43.6 percent; $\mathrm{Th}=0.26$ to 11.3 percent.

Color: Black, brown, yellowish brown. In granitic rocks, placers.

Dana VII, v. 1, p. 774-775.

Am. Mineralogist, v. 39, p. 109-117 (1954); v. 42, p. 30-38 (1957).

Absite

$2 \mathrm{UO}_{3} \cdot \mathrm{ThO}_{2} \cdot 7 \mathrm{TiO}_{2} \cdot 5 \mathrm{H}_{2} \mathrm{O}$

Thorian brannerite.

$\mathrm{U}=32.1$ percent; $\mathrm{Th}=\mathbf{1 5 . 6}$ percent.

Color: Yellow, yellow brown, brownish olive green.

In granodiorite and quartz veins.

Am. Mineralogist, v. 41, p. 166 (1956).

Cordobaite

Synonym of brannerite.

U.S. Geol. Survey Bull. 1064, p. 333-337 (1958).

Lodochnikite

Synonym of brannerite.

Am. Mineralogist, v. 43, p. 380, 1007 (1958); v. 48, p. 1419-1420 (19639

See also Thorutite, p. 40.

268-008-67—3 


\section{BROCKITE}

$(\mathrm{Ca}, \mathrm{Th}) \mathrm{PO}_{4} \cdot \mathrm{H}_{2} \mathrm{O}$

$\mathrm{Th}=36.8$ percent.

Color: Red and yellow. Occurs in veins in granitic rocks.

Am. Mineralogist, v. 47, p. 1346-1355 (1962).

\section{CALCIOSAMARSKITE}

Probably (Ca, Y, Ce, U, Th) ${ }_{3}(\mathrm{Nb}, \mathrm{Ta}, \mathrm{Fe}, \mathrm{Ti}, \mathrm{Sn})_{5} \mathrm{O}_{15}(?)$

$\mathrm{U}=9.4$ to 11.3 percent; $\mathrm{Th}=1.9$ to 2.9 percent.

Color: Black to brown. Rare, from granite pegmatite.

Dana VII, v. 1, p. 772.

\section{CALCIOURACONITE}

Near $\mathrm{Ca}\left(\mathrm{UO}_{2}\right)_{4}\left(\mathrm{SO}_{4}\right)_{2}(\mathrm{OH})_{6} \cdot 20 \mathrm{H}_{2} \mathrm{O}$

Validity questionable.

$\mathrm{U}=53.7$ percent.

Boldyrev, A. K., Course of descriptive mineralogy, v. 3 (1935), Leningrad and Moscow.

\section{CALCURMOLITE}

$\mathrm{Ca}\left(\mathrm{UO}_{2}\right)_{3}\left(\mathrm{MoO}_{4}\right)_{3}(\mathrm{OH})_{2} \cdot 11 \mathrm{H}_{2} \mathrm{O}$

$\mathrm{U}=39.4$ percent.

Color: Honey yellow. A secondary mineral.

Am. Mineralogist, v. 44, p. 468 (1959); v. 49, 1152 (1964).

\section{CARNOTITE}

$\mathrm{K}_{2}\left(\mathrm{UO}_{2}\right)_{2}\left(\mathrm{VO}_{4}\right)_{2} \cdot 3 \mathrm{H}_{2} \mathrm{O}$

$\mathrm{H}_{2} \mathrm{O}$ can range from 1 to 3 .

$\mathrm{U}=52.8$ to 55.0 percent.

Color: Yellow. Common secondary mineral.

Dana VII, v. 2, p. 1043-1045.

Am. Mineralogist, v. 39, p. 323 (1954).

\section{CARYOCERITE}

A borosilicate of $\mathrm{Ce}, \mathrm{Y}$, and $\mathrm{Th}$ chiefly.

$\mathrm{U}=$ trace; $\mathrm{Th}=12.0$ percent.

Color: Brown. From syenite pegmatite.

Dana VI, p. 415.

Heinrich, E. W., Mineralogy and geology of radioactive raw materials, 1958, p. 148.

\section{CERIANITE}

$(\mathrm{Ce}, \mathrm{Th}) \mathrm{O}_{2}$

$\mathrm{Ce}: \mathrm{Th}=$ about $16: 1$.

Isostructural with thorianite and uraninite.

$\mathrm{Th}=$ about 4.5 percent.

Am. Mineralogist, v. 46, p. 560-564 (1955).

U.S. Geol. Survey Bull. 1064, p. 53-55 (1958).

\section{CERPHOSPHORHUTTONITE}

$(\mathrm{Th}, \mathrm{Ce})(\mathrm{Si}, \mathrm{P}) \mathrm{O}_{4} \cdot 1.5 \mathrm{H}_{2} \mathrm{O}$, with $\mathrm{Si}$ slightly greater than $\mathrm{P}$.

$\mathrm{Th}=35.5$ percent; $\mathrm{U}=1.4$ percent.

Color: Pale yellow to reddish brown. Occurs in amazonite pegmatite. Am. Mineralogist, v. 50, p. 2099 (1965). 


\section{CHERALITE}

Phosphate-silicate of $\mathrm{Th}, \mathrm{Ca}$, and rare earths.

Isostructural with monazite, conforming to monazite formula type $A X \mathrm{O}_{4}$, with $A=\mathrm{Th}, \mathrm{Ca}, \mathrm{Ce}, \mathrm{La}, \mathrm{U}, \mathrm{Pb} ; X=\mathrm{P}, \mathrm{Si}$.

Is essentially an intermediate member of a solid solution series apparently extending between monazite, $\mathrm{CePO}_{4}$, and $\mathrm{CaTh}\left(\mathrm{PO}_{4}\right)_{2}$, known as an artificial compound.

$\mathrm{U}=3.5$ to 5.5 percent; $\mathrm{Th}=25.9$ to 27.7 percent.

Color: Green. Rare, from granite pegmatitie.

Mineralog. Mag., v. 30, p. 93-99 (1953).

Am. Mineralogist, v. 39, p. 403 (1954).

CHEVKINITE (or tscheffkinite)

$(\mathrm{Ce}, \mathrm{Y}, \mathrm{Ca}, \mathrm{U}, \mathrm{Th})_{2}(\mathrm{Ti}, \mathrm{Fe}, \mathrm{Mg})_{2}(\mathrm{Si}, \mathrm{Al})_{2} \mathrm{O}_{11}(?)$

$\mathrm{U}=\mathrm{up}$ to 2.3 percent; $\mathrm{Th}=$ up to 18.4 percent.

Color: Black. Accessory mineral in igneous rocks.

Am. Mineralogist, v. 31, p. 582-588 (1946); v. 41, p. 474-487 (1956); v. 44, p. 115-137 (1959).

See also Perrierite, p. 31.

\section{CLARKEITE}

$\left(\mathrm{Na}, \mathrm{Ca}, \mathrm{Pb}, \mathrm{Th}, \mathrm{H}_{2} \mathrm{O}\right)_{2} \mathrm{U}_{2}\left(\mathrm{O}, \mathrm{H}_{2} \mathrm{O}\right)_{7}$

$\mathrm{U}=64.2$ to 68.0 percent; $\mathrm{Th}=2.2$ percent.

Color: Brown, reddish brown. Alteration product of uraninite.

Am. Mineralogist, v. 39, p. 826-838 (1954); v. 41, p. 127-133 (1956).

U.S. Geol. Survey Bull. 1064, p. 95-98 (1958).

\section{COCONINOITE}

$\mathrm{Fe}_{2}{ }^{+3} \mathrm{Al}_{2}\left(\mathrm{UO}_{2}\right)_{2}\left(\mathrm{PO}_{4}\right)_{4}\left(\mathrm{SO}_{4}\right)(\mathrm{OH})_{2} \cdot 20 \mathrm{H}_{2} \mathrm{O}$

$\mathrm{U}=28.5$ to 29.0 percent.

Color: Light creamy yellow. A secondary mineral.

Am. Mineralogist, v. 51, p. 651-663 (1966).

\section{COFFINITE}

$\mathrm{U}\left(\mathrm{SiO}_{4}\right)_{1-x}(\mathrm{OH})_{4 x}$

$\mathrm{U}=40.9$ to 60.2 percent in concentrated, but not pure, samples.

Color: Black very fine particles ( $-325 \mathrm{mesh})$, pale brown in transmitted light. Occurs in many deposits in sandstones and in hydrothermal veins.

Am. Mineralogist, v. 41, p. 675-688 (1956).

See also Nenadkevite, p. 29.

COMPREIGNACITE

$\mathrm{K}_{2} \mathrm{O} \cdot 6 \mathrm{UO}_{3} \cdot 11 \mathrm{H}_{2} \mathrm{O}$

Potassium analog of billietite.

$\mathrm{U}=71.0$ percent.

Color: Yellow. A secondary mineral.

Soc. Française, Mineralogie et Cristallographie Bull., v. 87, p. 365-371 (1964).

Am. Mineralogist, v. 50, p. $807-808$ (1965).

\section{COUSINITE}

$\mathrm{MgO} \cdot 2 \mathrm{MoO}_{3} \cdot 2 \mathrm{UO}_{2} \cdot 6 \mathrm{H}_{2} \mathrm{O}$

Description of mineral inadequate. May possibly be moluranite.

$\mathrm{U}=48.8$ percent.

Color: Black. A secondary mineral.

Am. Mineralogist, v. 44, p. 910 (1959). 
CUPROSKLODOWSKITE (or cuprosklowskite)

$\mathrm{Cu}\left(\mathrm{UO}_{2}\right)_{2}\left(\mathrm{SiO}_{3}\right)_{2}(\mathrm{OH})_{2} \cdot 5 \mathrm{H}_{2} \mathrm{O}$

Isostructural with sklodowskite and uranophane.

$\mathrm{U}=54.1$ percent.

Color: Greenish yellow. A secondary mineral.

Am. Mineralogist, v. 19, p. 235 (1934).

U.S. Geol. Survey Bull. 1064, p. 304-307 (1958).

Jáchymovite

Synonym of cuprosklodowskite.

Mineralog. Abs., v. 6, p. 345 (1936).

Kieselkupfer-uranoxyd

Synonym of cuprosklodowskite.

Neues. Jahrb. Mineralogie, 1845, p. 297.

CUPROZIPPEITE

$\mathrm{Cu}\left(\mathrm{UO}_{2}\right)_{2}\left(\mathrm{SO}_{4}\right)_{2}(\mathrm{OH})_{2} \cdot 11 \mathrm{H}_{2} \mathrm{O}$

Validity questionable.

Boldyrev, A. K., Course of descriptive mineralogy, v. 3, p. 83 (1935), Leningrad and Moscow.

CURITE

$3 \mathrm{PbO} \cdot 8 \mathrm{UO}_{3} \cdot 5 \mathrm{H}_{2} \mathrm{O}$

$\mathrm{U}=61.8$ to 62.6 percent.

Color: Orange red. An alteration product of uraninite.

Dana VII, v. 1, p. 629-631.

Soc. Française Minéralogie et Cristallographie Bull. v. 78, p. 1-26 (1955); v. 82 , p. $258-260$ (1959).

DAVIDITE

$A_{6} B_{15}(\mathrm{O}, \mathrm{OH})_{36}$, where $A=\mathrm{Fe}^{+2}$, rare earths, U, Ca, $\mathrm{Zr}$, Th; $B=\mathrm{Ti}, \mathrm{Fe}^{+3}$, $\mathrm{V}, \mathrm{Cr}$.

Ideal end member is $\mathrm{FeTi}_{3} \mathrm{O}_{7}$.

$\mathrm{U}=$ up to 4.4 percent; $\mathrm{Th}=\mathrm{up}$ to 0.12 percent.

Color: Black. A primary mineral in high-temperature hydrothermal lodes.

Mineralog. Mag., v. 29, p. 101 (1950).

Am. Mineralogist, v. 36 , p. $563-572$ (1951); v. 45 , p. $700-718$ (1961).

U.S. Geol. Survey Bull. 1064, p. 337-341 (1958).

Econ. Geology, v. 54, p. 64-81 (1959).

Ferutite

Synonym of davidite.

$\mathrm{U}=12.5$ percent; $\mathrm{Th}=0.06$ percent.

Color: Black. A detrital mineral.

Mineralog. Mag., v. 29, p. 101-112 (1950).

Zapiski Vses. Mineralogie Obshch., v. 83, no. 4, p. 425 (1954).

Am. Mineralogist, v. 43, p. 382-383 (1958); v. 49, p. 447 (1964).

Mavudzite

Probably the same as ferutite.

Am. Mineralogist, v. 41, p. 164 (1956).

Ufertite

Synonym of davidite.

$\mathrm{U}=2.3$ percent; $\mathrm{Th}=0.14$ to 0.18 percent.

Color: Brownish-black. Occurs in biotite gneisses and quartz-biotite schists.

Am. Mineralogist, v. 43, p. 378-379 (1958); v. 49, p. 447 (1964). 
DAVIDITE-Continued

Unnamed mineral from Tête district of Mozambique

Possibly $A B_{3}(\mathrm{O}, \mathrm{OH})_{7}$, where $A=\mathrm{Fe}^{+2}$, rare earths, $\mathrm{U}^{+6}, \mathrm{Ca}, \mathrm{Na}, \mathrm{Zr}$, $\mathrm{Th} ; B=\mathrm{Ti}, \mathrm{Fe}^{+3}, \mathrm{~V}, \mathrm{Cr}$.

Probably davidite.

$\mathrm{U}=2.7$ percent; $\mathrm{Th}=0.06$ to 0.12 percent.

Mineralog. Mag., v. 29, p. 101-112 (1950).

DEMESMAEKERITE

$\mathrm{Pb}_{2} \mathrm{Cu}_{5}\left(\mathrm{UO}_{2}\right)_{2}\left(\mathrm{SeO}_{3}\right)_{6}(\mathrm{OH})_{6} \cdot 2 \mathrm{H}_{2} \mathrm{O}$

$\mathrm{U}=22.5$ percent.

Color: Bottle green to clear olive green. Occurs in lower part of oxidation zone.

Soc. Française Minéralogie et Cristallographie Bull., v. 88 , p. $422-425$ (1965).

\section{DEWINDTITE}

$\mathrm{Pb}_{3}\left(\mathrm{UO}_{2}\right)_{5}\left(\mathrm{PO}_{4}\right)_{4}(\mathrm{OH})_{4} \cdot 10 \mathrm{H}_{2} \mathrm{O}(?)$

Isostructural with phosphuranylite and renardite; possibly identical with renardite.

$\mathrm{U}=49.5$ percent.

Color: Canary yellow. A rare secondary mineral.

Dana VII, v. 2, p. 875 .

Am. Mineralogist, v. 35, p. 756-763 (1950); v. 39, p. 444-451 (1954); v. 41 , p. 921 (1956).

Stasite

Synonym of dewindtite.

DROOGMANSITE

No chemical analysis has been made.

May be related to sklodowskite.

Am. Mineralogist, v. 11, p. 168 (1926).

DUMONTITE

$\mathrm{Pb}_{2}\left(\mathrm{UO}_{2}\right)_{3}\left(\mathrm{PO}_{4}\right)_{2}(\mathrm{OH})_{4} \cdot 3 \mathrm{H}_{2} \mathrm{O}$

$\mathrm{U}=46.5$ percent.

Color: Yellow. A rare secondary mineral.

Dana VII, v. 2, p. 928-929.

\section{EKANITE}

$(\mathrm{Th}, \mathrm{U})(\mathrm{Ca}, \mathrm{Fe}, \mathrm{Pb})_{2} \mathrm{Si}_{8} \mathrm{O}_{20}$

$\mathrm{U}=1.8$ percent; $\mathrm{Th}=24.0$ percent.

Color: Green. Occurs in gem-bearing gravel, Ceylon.

Nature, v. 190 , no. 4780 , p. 997 (1961).

Am. Mineralogist, v. 46, p. 1516 (1961).

ESCHYNITE. See Aeschynite, p. 10.

\section{EUXENITE}

$(\mathrm{Y}, \mathrm{Ca}, \mathrm{Ce}, \mathrm{U}, \mathrm{Th})(\mathrm{Nb}, \mathrm{Ta}, \mathrm{Ti})_{2} \mathrm{O}_{6}$

Part of the euxenite-polycrase series.

$\mathrm{U}=\mathbf{0 . 6}$ to 12.45 percent; $\mathrm{Th}=$ up to $\mathbf{1 4 . 2}$ percent.

Color: Black. From granite pegmatites.

Dana VII, v. 1, p. 787-791.

Geol. Soc. America Bull., v. 61, p. 129-132 (1950).

Mineralog. Mag., v. 31, p. 763-780 (1958). 


\section{EUXENITE-Continued}

Delorenzite

Originally described as $(\mathrm{Y}, \mathrm{U}, \mathrm{Fe})(\mathrm{Ti}, \mathrm{Sn})_{3} \mathrm{O}_{8}$, but recently shown to be tanteuxenite.

$\mathrm{U}=6.6$ percent.

Dana VII, v. 1, p. 808.

Mineralog. Mag., v. 32, p. 308-313 (1959).

Eschwegeite

Tantalian variety of euxenite.

Am. Mineralogist, v. 36, p. 927 (1951).

Oliveiraite

Alteration product of euxenite.

Dana VII, v. 1, p. 791.

Tanteuxenite

Variety of euxenite with Ta substituting for $\mathrm{Nb}$.

Dana VII, v. 1, p. 787, 789-790.

Am. Mineralogist, v. 35, p. 386-400 (1950).

Titanoniobite

Allied to euxenite.

Mineralog. Abs., v. 11, p. 232 (1951).

FENGHUANGLITE (or Feng-huang-shih)

An unnecessary name for thorium-rich britholite.

$(\mathrm{Ca}, \mathrm{Ce}, \mathrm{La}, \mathrm{Th})_{5}\left[(\mathrm{Si}, \mathrm{P}, \mathrm{C}) \mathrm{O}_{4}\right]_{3}(\mathrm{O}, \mathrm{OH})(?)$

$\mathrm{Th}=17.3$ percent.

Color: Yellowish brown. From nepheline hyroxenite.

Am. Mineralogist, v. 45, p. 754-755 (1960); v. 48, p. 211.

Fynchenite

Russian transliteration of Feng-huang-shih.

FER GHANITE

Possibly $\left(\mathrm{UO}_{2}\right)_{3}\left(\mathrm{VO}_{4}\right)_{2} \cdot 6 \mathrm{H}_{2} \mathrm{O}$

Perhaps leached or weathered tyuyamunite. Poorly defined; needs further study.

$\mathrm{U}=62.2$ percent.

Color: Yellow. A secondary mineral.

Dana VII, v. 2, p. 1048.

U.S. Geol. Survey Bull. 1064, p. 260-281 (1958).

FERGUSONITE

(Y, Er, Ce,Fe) (Nb,Ta,Ti) $\mathrm{O}_{4}$

Part of the fergusonite-formanite series.

$\mathrm{U}=0.8$ to 7.2 percent; $\mathrm{Th}=0.7$ to 6.0 percent.

Color: Black; alters to brown and yellow. From granite pegmatites.

Dana VII, v. 1, p. 757-762.

Soc. Française Minéralogie et Cristallographie Bull., v. 81, p. 338 (1958)

Mineralog. Mag., v. 31, p. 763-780 (1958).

Adelpholite

Synonym of fergusonite(?)

A poorly defined substance, possibly an altered mossite.

Dana VII, v. 1, p. 762, 778-779. 
FERGUSONITE-Continued

Alpha-fergusonite

Tetragonal fergusonite.

Arrhenite

An altered fergusonite.

Dana VII, v. 1, p. 762 .

Beta-fergusonite

Monoclinic fergusonite.

Bragite

Synonym of fergusonite.

Dana VII, v. 1, p. 757, 759, 761.

Kochelite

Synonym of fergusonite.

Dana VII, v. 1, p. 757, 761.

Risörite

Synonym of fergusonite.

Dana VII, v. 1, p. 758-759, 760-762.

Rutherfordite (not the same as Rutherfordine)

An altered fergusonite.

Dana VII, v. 1, p. 757, 759-760, 762.

Sipylite

Synonym of fergusonite.

Dana VII, v. 1, p. 757, 759-760, 762.

Tyrite

Synonym of fergusonite.

Dana VII, v. 1, p. 757, 760-761.

FORMANITE

(Y,U,Th, Ca) (Ta, Nb, Ti) $\mathrm{O}_{4}$

Part of the fergusonite-formanite series.

$\mathrm{U}=1.1$ percent; $\mathrm{Th}=1.1$ percent.

Color: Black. From placers.

Dana VII, v. 1, p. 758, 760, 762.

Mineralog. Mag., v. 31, p. 763-780 (1958).

FOURMARIERITE

$\mathrm{PbO} \cdot 4 \mathrm{UO}_{3} \cdot 5 \mathrm{H}_{2} \mathrm{O}$

$\mathrm{U}=64.6-65.3$ percent.

Color: Orange red. A rare secondary mineral.

Am. Mineralogist, v. 33, p. 619-621 (1948).

Soc. Française Minéralogie et Cristallographie Bull., v. 82, p. 260-261 (1958).

\section{FRANCEVILLITE}

$(\mathrm{Ba}, \mathrm{Pb})\left(\mathrm{UO}_{2}\right)_{2}\left(\mathrm{VO}_{4}\right)_{2} \cdot 5 \mathrm{H}_{2} \mathrm{O}$

Apparently the barium analog of meta-tyuyamunite.

$\mathrm{U}=45.8$ percent.

Color: Yellow. A secondary mineral; occurs in sandstone.

Am. Mineralogist, v. 43, p. 180 (1958). 


\section{FRITZSCHEITE}

$\mathrm{Mn}\left(\mathrm{UO}_{2}\right)_{2}\left[(\mathrm{P}, \mathrm{V}) \mathrm{O}_{4}\right]_{2} \cdot 8 \mathrm{H}_{2} \mathrm{O}(?)$

No analysis available. May be the manganese analog of torbernite.

Color: Reddish brown to hyacinth red. A rare secondary mineral.

Dana VII, v. 2, p. 984 .

U.S. Geol. Survey Bull. 1064, p. 195-196 (1958).

\section{GRAYITE}

A thorium phosphate, no analysis given, with structure like that of rhabdophane, perhaps ( $\mathrm{Th}, \mathrm{Pb}, \mathrm{Ca}$ ) $\mathrm{PO}_{4} \cdot \mathrm{H}_{2} \mathrm{O}$

Color: Yellow, powdery. From pegmatite.

Great Britain Geol. Survey, Summary of Progress for 1956, p. 67 (1957).

Am. Mineralogist, v. 47, p. 419-420 (1962).

Compare Ningyoite, p. 29.

\section{GUILLEMINITE}

$\mathrm{Ba}\left(\mathrm{UO}_{2}\right)_{3}(\mathrm{OH})_{4}\left(\mathrm{SeO}_{3}\right)_{2} \cdot 3 \mathrm{H}_{2} \mathrm{O}$

$\mathrm{U}=53.8$ percent.

Color: Canary yellow. A secondary mineral.

Soc. Française Mineralogie et Crystallographic Bull., v. 88, p. 132-135 (1965).

Am. Mineralogist, v. 50, p. 2103 (1965).

GUMMITE

Generic term for minerals occurring as alteration product of uraninite and not otherwise identified.

Group includes silicates, phosphates, and oxides.

Dana VII, v. 1, p. 622-625.

Am. Mineralogist, v. 41, p. 539-568 (1956).

Eliasite

Synonoym of gummite.

$\mathrm{U}=57.2$ percent.

Dana VII, v. 1, p. 622-624.

Pittinite

Synonym of gummite.

Dana VII, v. 1, p. 622-624.

Yttrogummite

Yttrian variety of gummite.

An alteration product of yttrian uraninite.

Dana VII, v. 1, p. 622-624.

See also Urgite, p. 46.

\section{HAIWEEITE}

$\mathrm{CaO} \cdot 2 \mathrm{UO}_{3} \cdot 6 \mathrm{SiO}_{2} \cdot 5 \mathrm{H}_{2} \mathrm{O}$

State of hydration uncertain.

$\mathrm{U}=52.8$ percent.

Color: Pale yellow to greenish yellow. A secondary mineral.

Am. Mineralogist, v. 441, p. 839-843 (1959).

Gastunite

Synonym of haiweeite.

U.S. Geol. Survey Bull. 1064, p. 311-312 (1958).

Am. Mineralogist, v. 45, p. 52 (1960).

Neues Jahrb. Mineralogie Monatsh. 1960, p. 37-47. 


\section{HALLIMONDITE}

$\mathrm{Pb}_{2}\left(\mathrm{UO}_{2}\right)\left(\mathrm{AsO}_{4}\right)_{2}$

$\mathrm{U}=28.1$ percent.

Color: Yellow. A secondary mineral.

Am. Mineralogist, v. 50, p. 1143-1157 (1965).

\section{HEINRICHITE}

$\mathrm{Ba}\left(\mathrm{UO}_{2}\right)_{2}\left(\mathrm{AsO}_{4}\right)_{2} \cdot 10-12 \mathrm{H}_{2} \mathrm{O}$

Formula assumed on basis of similarity of X-ray powder pattern to that of zeunerite.

$\mathrm{U}=\mathbf{4 0 . 7}$ to $\mathbf{4 1 . 9}$ percent.

Color: Yellow to green. A secondary mineral.

Am. Mineralogist, v. 43, p. 1134-1147 (1958).

Sandbergerite

Synonym of heinrichite.

Am. Mineralogist, v. 43, p. 1135 (1958).

HIELMITE (or hjelmite)

$A B_{2} \mathrm{O}_{8}$ or $A_{2} B_{3} \mathrm{O}_{10}$, where $A=\mathrm{Y}, \mathrm{Fe}^{+2}, \mathrm{U}^{+4}, \mathrm{Mn}, \mathrm{Ca} ; B=\mathrm{Nb}, \mathrm{Ta}, \mathrm{Sn}, \mathrm{W}$. $\mathrm{U}=4.0$ to 4.3 percent.

Color: Black. From pegmatite.

Dana VII, v. 1, p. 779-780.

\section{HƯGELITE}

$\mathrm{Pb}_{2}\left(\mathrm{UO}_{2}\right)_{3}\left(\mathrm{AsO}_{4}\right)_{2}(\mathrm{OH})_{4} \cdot 3 \mathrm{H}_{2} \mathrm{O}$

No analysis, formula by analogy to dumontite, with which it is isostructural.

Color: Brown to orange yellow. A secondary mineral.

Am. Mineralogist, v. 47, p. 418-419 (1962).

\section{HUTTONITE}

\section{$\mathrm{ThSiO}_{4}$}

Dimorph of thorite, isostructural with monazite and cheralite.

$\mathrm{Th}=71.6$ percent.

Color: Colorless to pale cream. Rare mineral from granitic pegmatites, alaskite granite, and placers.

Am. Mineralogist, v. 36, p. 60-69 (1951).

See also Cerphosphorhuttonite, p. 14.

\section{HYDROCERITE}

$(\mathrm{La}, \mathrm{Ce}, \mathrm{Th})_{2}(\mathrm{Si}, \mathrm{P})_{2} \mathrm{O}_{7} \cdot 5 \mathrm{H}_{2} \mathrm{O}$

Amorphous, gives a ceritelike $\mathrm{X}$-ray pattern when heated, and is perhaps related to rhabdophane.

$\mathrm{Th}=13.3$ percent.

Color: Yellow to honey yellow. Occurs in alkalic pegmatite.

Am. Mineralogist, v. 45, p. 1132 (1960); v. 47, p. 419-420 (1962).

\section{HYDROGEN AUTUNITE}

$\mathrm{H}_{2}\left(\mathrm{UO}_{2}\right)_{2}\left(\mathrm{PO}_{4}\right)_{2} \cdot 8-10 \mathrm{H}_{2} \mathrm{O}$

$\mathrm{U}=51.7$ to 54.3 percent.

Color: Pale yellow. Rare secondary mineral.

United Nations Internat. Conf. on Peaceful Uses of Atomic Energy, 2d, Geneva, Proc., v. 2, p. 298-299 (1958). 
HYDRONASTURAN

$\mathrm{UO}_{2} \cdot k \mathrm{UO}_{3} \cdot n \mathrm{H}_{2} \mathrm{O}$

$k=2.3$ to $5.0 ; n=3.9$ to 9.0 percent.

Of doubtful validity, presumably an altered pitchblende.

$\mathrm{U}=10.5$ to 45.0 percent.

Color: Black to bottle green. A secondary mineral.

Am. Mineralogist, v. 42, p. 442-443 (1957).

IANTHINITE

$\mathrm{UO}_{2} \cdot 5 \mathrm{UO}_{3} \cdot 10-11 \mathrm{H}_{2} \mathrm{O}$

This has been known also as "ianthinite français," and is not to be confused with "ianthinite," redescribed as wyartite.

$\mathrm{U}=75.6$ percent.

Color: Violet black. Rare secondary mineral.

Soc. Française Minéralogie et Cristallographie Bull., v. 82 , p. 80-86. (1959).

Am. Mineralogist. v. 44, p. 1103-1104 (1959).

\section{IRIGINITE}

$\mathrm{UO}_{3} \cdot 2 \mathrm{MoO}_{3} \cdot 3 \mathrm{H}_{2} \mathrm{O}$ or $\mathrm{U}\left(\mathrm{MoO}_{4}\right)_{2}(\mathrm{OH})_{2} \cdot 2 \mathrm{H}_{2} \mathrm{O}$

$\mathrm{U}=38.7$ percent.

Color: Canary yellow. A rare secondary mineral.

Am. Mineralogist, v. 43, p. 379 (1958); v. 45, p. 257-258 (1960); v. 49 p. 408-414 (1964).

\section{ISHIKAWAITE}

$(\mathrm{U}, \mathrm{Fe}, \mathrm{Y}, \mathrm{Ce})(\mathrm{Nb}, \mathrm{Ta}) \mathrm{O}_{4}$

$\mathrm{U}=\mathbf{1 9 . 3}$ percent.

Color: Black. Rare, from granite pegmatites.

Dana VII, v. 1, p. 766.

\section{JOHANNITE}

$\mathrm{Cu}\left(\mathrm{UO}_{2}\right)_{2}\left(\mathrm{SO}_{4}\right)_{2}(\mathrm{OH})_{2} \cdot 6 \mathrm{H}_{2} \mathrm{O}$

$\mathrm{U}=50.8$ percent.

Color: Green. A secondary mineral.

Dana VII, v. 2, p. 606-607.

\section{Gilpinite}

Synonym of johannite.

Am. Mineralogist, v. 11, p. 1-5 (1926).

\section{Peligotite}

$\mathrm{CuO} \cdot 2 \mathrm{UO}_{3} \cdot 2 \mathrm{SO}_{3} \cdot 3 \mathrm{H}_{2} \mathrm{O}$

Probably the same as johannite.

$\mathrm{U}=45.1$ percent.

Color: Green yellow. A secondary mineral.

Am. Mineralogist, v. 40, p. 369 (1955).

U.S. Geol. Survey Bull., 1064, p. 135 (1958).

Uranvitriol

Synonym of johannite.

Chem. Untersuch., v. 5, p. 254 (1821).

\section{KAHLERITE}

$\mathrm{Fe}\left(\mathrm{UO}_{2}\right)_{2}\left(\mathrm{AsO}_{4}\right)_{2} \cdot n \mathrm{H}_{2} \mathrm{O}$

Formula assumed from qualitative tests. 
KAHLERITE-Continued

Arsenate analogue of bassetite. Needs further study.

$\mathrm{U}=46.8$ percent(?).

Color: Yellow to yellow green. A rare secondary mineral.

Der Karinthin, v. 23, p. 277-280 (1953).

Am. Mineralogist, v. 39, p. 1038 (1954).

\section{KASOLITE}

$\mathrm{Pb}\left(\mathrm{UO}_{2}\right)\left(\mathrm{SiO}_{3}\right)(\mathrm{OH})_{2}$

$\mathrm{U}=40.5$ percent.

Color: Yellow to brown. A secondary mineral.

Am. Mineralogist, v. 7, p. 128-129 (1922).

Acad. Royale Belgique Bull. Cl. Sci., v. 25, p. 654 (1939).

Compare Orlite, p. 30.

KHLOPINITE (or chlopinite, or hlopinite)

$\left(\mathrm{Y}, \mathrm{U}^{+4}, \mathrm{Th}\right)_{3}(\mathrm{Nb}, \mathrm{Ta}, \mathrm{Ti}, \mathrm{Fe})_{7} \mathrm{O}_{20}(?)$

May be related to euxenite-polycrase.

$\mathrm{U}=7.2$ percent; $\mathrm{TH}=1.9$ percent.

Color: Black. From pegmatites.

Dana VII, v. 1., p. 792.

\section{KIV UITE}

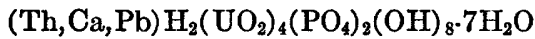

Stated to be the thorium analog of phosphuranylite. Needs further study.

$\mathrm{U}=52.3$ percent; $\mathrm{Th}=7.3$ percent.

Color: Yellow. Earthy. From pegmatites.

Am. Mineralogist, v. 44, p. 1326-1327 (1959).

\section{KOBEITE}

Perhaps $A B_{2}(\mathrm{O}, \mathrm{OH})_{6}$

$A=\mathrm{Y}, \mathrm{U} ; B=\mathrm{Ti}$ mainly, $\mathrm{Fe}^{+3}, \mathrm{Nb}$, Ta.

$\mathrm{U}=8.4$ to 10.7 percent; $\mathrm{Th}=0.7$ to 1.0 percent.

Color: Black. From a pegmatite.

Am. Mineralogist, v. 36, p. 925 (1951); v. 42, p. 342-353 (1957).

\section{LERMONTOVITE}

$\left(\mathrm{U}, \mathrm{Ca}, \mathrm{R} . \mathrm{E} \cdot{ }^{*}\right)_{3}\left(\mathrm{PO}_{4}\right)_{4} \cdot 6 \mathrm{H}_{2} \mathrm{O}$

Needs further study. Possibly identical with ningyoite.

$\mathrm{U}=44.3$ percent.

Color: Grayish green. Occurs in zone of cementation of hydrothermal deposits.

Am. Mineralogist, v. 43, p. $379-380^{\circ}(1958)$.

\section{LIEBIGITE}

$\mathrm{Ca}_{2}\left(\mathrm{UO}_{2}\right)\left(\mathrm{CO}_{3}\right)_{3} \cdot 10 \mathrm{H}_{2} \mathrm{O}$

$\mathrm{U}=33.5$ percent.

Color: Green, yellow green. Rare secondary mineral.

Dana VII, v. 2, p. 240.

Am. Mineralog ist, v. 35, p. 251-254 (1950).

U.S. Geol. Survey Bull. 1064, p. 108-112 (1958).

Flutherite

Synonym of liebigite.

- Rare earths. 
LIEBIGITE-Continued

Kalk-uran-carbonat

Synonym of liebigite.

U.S. Geol. Survey Bull. 1064, p. 108 (1958).

Uranothallite

Synonym of liebigite.

Dana VII, v. 2, p. 241.

MACKELVEYITE (or mckelveyite)

Near $\mathrm{Na}_{2} \mathrm{Ba}_{4} \mathrm{Ca}(\mathrm{Y}, \mathrm{U})_{2}\left(\mathrm{CO}_{3}\right)_{9} \cdot 5 \mathrm{H}_{2} \mathrm{O}$

$\mathrm{U}=3.9$ percent; $\mathrm{Th}=0.09$ percent.

Color: Dark green or black; rarely apple green. A secondary mineral.

Am. Mineralogist, v. 50, p. 593-612 (1965).

MASUYITE

$\mathrm{UO}_{3} \cdot 2 \mathrm{H}_{2} \mathrm{O}$

Probably isostructural with vandendriesscheite. Analysis of authentic material yielded no lead.

$\mathrm{U}=73.9$ percent.

Color: Yellow to orange yellow. Rare secondary mineral.

U.S. Geol. Survey Bull. 1064, p. 78-81 (1958).

Am. Mineralogist, v. 45, p. 1026-1061 (1960).

MEDJIDITE

Supposedly a uranium sulfate.

Validity of species is questionable.

Dana VII, v. 2, p. 600.

META-ANKOLEIITE

$\mathrm{K}_{2}\left(\mathrm{UO}_{2}\right)_{2}\left(\mathrm{PO}_{4}\right)_{2} \cdot 6 \mathrm{H}_{2} \mathrm{O}$

$\mathrm{U}=49.6$ to 50.6 percent.

Color: Yellow. A rare secondary mineral found in granitic pegmatite and in sandstone.

Am. Mineralogist, v. 52, p. 560 (1967).

META-A UTUNITE I

$\mathrm{Ca}\left(\mathrm{UO}_{2}\right)_{2}\left(\mathrm{PO}_{4}\right)_{2} \cdot 2-6 \mathrm{H}_{2} \mathrm{O}$

Apparently not formed directly in nature, but most field and museum specimens of autunite have been dehydrated to this phase.

$\mathrm{U}=53$ to 59 percent.

Color: Yellow. A common secondary mineral.

Dana VII, v. 2, p. 985.

Soc. Française Minéralogie et Cristallographie Bull., v. 81, p. 4-10 (1958).

Am. Mineralogist, v. 45, p. 99-128 (1960); v. 48, p. 1389-1393 (1963).

See also Autunite, p.11.

Metakalkuranite

Synonym of meta-autunite.

Centralbl. Mineralogie, 1901, p. 709.

META-A UTUNITE II

$\mathrm{Ca}\left(\mathrm{UO}_{2}\right)_{2}\left(\mathrm{PO}_{4}\right)_{2} \cdot 0-6 \mathrm{H}_{2} \mathrm{O}$

Not found in nature. Meta-autunite I passes into this phase on heating to about $80^{\circ} \mathrm{C}$.

$\mathrm{U}=53.0$ to 61.8 percent.

Color: Yellow. 


\section{META-AUTUNITE II-Continued}

Dana VII, v. 2, p. 985.

Soc. Française Minéralogie et Cristallographie Bull. v. 81, p. 4-10 (1958).

Am. Mineralogist, v. 45, p. 99-128 (1960).

See also Autunite p. 11; Meta-autunite I, above.

Para-autunite

$\mathrm{Ca}\left(\mathrm{UO}_{2}\right)_{2}\left(\mathrm{PO}_{4}\right)_{2}$

Completely dehydrated autunite. Equals meta-autunite II.

$\mathrm{U}=61.8$ percent.

Strunz, Hugo, Mineralogische Tabellen, 4th ed., 1966, p. 313; Leipzig, Akad. Verlag., 560 p.

METAHAIWEEITE

$\mathrm{CaO} \cdot 2 \mathrm{UO}_{3} \cdot 6 \mathrm{SiO}_{2} \cdot n \mathrm{H}_{2} \mathrm{O}$, where $n=$ less than 5 .

No analysis, apparently a dehydration product of haiweeite.

A secondary mineral.

Am. Mineralogist, v. 44, p. 839-843 (1959).

METAHEINRICHITE

$\mathrm{Ba}\left(\mathrm{UO}_{2}\right)_{2}\left(\mathrm{AsO}_{4}\right)_{2} \cdot 8 \mathrm{H}_{2} \mathrm{O}$

$\mathrm{U}=43.3$ percent.

Color: Yellow to green. A secondary mineral.

Am. Mineralogist, v. 43, p. 1134-1147 (1958).

Arsenuranocircite

Synonym of metaheinrichite.

Am. Mineralogist, v. 44, p. 466 (1959).

Metasandbergerite

Synonym of metaheinrichite.

Am. Mineralogist, v. 43, p. 1135 (1958).

METAKAHLERITE

$\mathrm{Fe}\left(\mathrm{UO}_{2}\right)_{2}\left(\mathrm{AsO}_{4}\right)_{2} \cdot 8 \mathrm{H}_{2} \mathrm{O}(?)$

No analysis made; microchemical tests showed Fe, As, U. X-ray powder pattern and optics indicate it is a meta form.

$\mathrm{U}=45.7$ percent.

Color: Sulfur yellow to yellowish green. A secondary mineral.

Geol. Landesamt Baden-Württemberg Jahresh., v. 3, p. 17-51 (1958).

Am. Mineralogist, v. 45, p. 254 (1960).

METAKIRCHHEIMERITE

$\mathrm{Co}\left(\mathrm{UO}_{2}\right)_{2}\left(\mathrm{AsO}_{4}\right)_{2} \cdot 8 \mathrm{H}_{2} \mathrm{O}$

Microchemical analysis shows presence of $\mathrm{Co}, \mathrm{U}$, and As.

$\mathrm{X}$-ray powder pattern shows it is a meta form.

$\mathrm{U}=46.6$ percent.

Color: Pale rose. A secondary mineral.

Am. Mineralogist, v. 44, p. 466 (1959).

METANOVACEKITE

$\mathrm{Mg}\left(\mathrm{UO}_{2}\right)_{2}\left(\mathrm{AsO}_{4}\right)_{2} \cdot 4 \mathrm{H}_{2} \mathrm{O}$

Partly dehydrated form of novacekite.

$\mathrm{U}=52.1$ percent.

Color: Yellow.

Strunz, Hugo, Mineralogischen Tabellen, 4th ed., 1966, p. 312; Leipzig, Akad. Verlag., 560 p.

Soc. Française Minéralogie et Cristallographie Bull., v. 81, p. 71 (1958). 
METASALEEITE

$$
\mathrm{Mg}\left(\mathrm{UO}_{2}\right)_{2}\left(\mathrm{PO}_{4}\right)_{2} \cdot 4 \mathrm{H}_{2} \mathrm{O}(?)
$$

$\mathrm{U}=65.1$ percent.

Color: Yellow. A secondary mineral.

Soc. Toscana Sci. Nat. Atti, ser. A, v. 55, p. 248-254 (1958).

\section{METATORBERNITE}

$\mathrm{Cu}\left(\mathrm{UO}_{2}\right)_{2}\left(\mathrm{PO}_{4}\right)_{2} \cdot n \mathrm{H}_{2} \mathrm{O}$, where $n=4(?)$ to 8 .

$\mathrm{U}=50.8$ to 55.0 percent.

Color: Green. A common secondary mineral.

Dana VII, v. 2, p. 991.

U.S Geol. Survey Bull. 1064, p. 298 (1958).

Am. Mineralogist, v. 49, p. 1603-1621 (1964).

See also Torbernite, p. 41.

Metachalcolite

Synonym of metatorbernite.

Dana VII, v. 2, p. 991.

Metakupferuranit

Synonym of metatorbernite.

Centralbl. Mineralogie, 1901, pl. 618.

METATYUYAMUNITE

$\mathrm{Ca}\left(\mathrm{UO}_{2}\right)_{2}\left(\mathrm{VO}_{4}\right)_{2} \cdot 3-5 \mathrm{H}_{2} \mathrm{O}$

Lower hydrate of tyuyamunite.

$\mathrm{U}=52.9$ to 55.1 percent.

Color: Yellow. A secondary mineral.

Am. Mineralogist, v. 41, p. 187-201 (1956).

META-URANOCIRCITE

\section{$\mathrm{Ba}\left(\mathrm{UO}_{2}\right)_{2}\left(\mathrm{PO}_{4}\right)_{2} \cdot 8 \mathrm{H}_{2} \mathrm{O}$}

Belongs to the meta-series of hydrates of the metatorbernite group and was known originally as uranocircite.

$\mathrm{U}=47.1$ percent.

Color: Yellow green. A secondary mineral.

Am. Mineralogist, v. 38, p. 476 (1953).

Soc. Française Minéralogie et Cristallographie Bull,. v. 80 , p. $32-38$ (1957).

Uranocircite

Now recognized as meta-uranocircite.

Am. Mineralogist, v. 38, p. 476 (1953).

Bariumphosphoruranit

Synonym of uranocircite.

Mineralog. Mag., v. 28, p. 732 (1949).

\section{META-URANOPILITE}

$\left(\mathrm{UO}_{2}\right)_{6}\left(\mathrm{SO}_{4}\right)(\mathrm{OH})_{10} \cdot 5 \mathrm{H}_{2} \mathrm{O}(?)$

Recent work indicates validity of species is doubtful.

$\mathrm{U}=72.3$ percent(?).

Dana VII, v. 2, p. 582-583.

Am. Mineralogist, v. 37, p. 958 (1954).

Soc. Française Minéralogie et Cristallographie Bull., v. 78 , p. 1-26 (1955).

See also Uranopilite, p. 45. 


\section{META-URANOPILITE-Continued}

Beta-uranopilite

Synonym of meta-uranopilite.

Dana VII, v. 2, p. 582.

META-URANOSPINITE

$\mathrm{Ca}\left(\mathrm{UO}_{2}\right)_{2}\left(\mathrm{AsO}_{4}\right)_{2} \cdot 8 \mathrm{H}_{2} \mathrm{O}$

It is uncertain whether this lower hydrate occurs naturally.

$\mathrm{U}=46.4$ percent.

Color: Yellow. A secondary mineral.

Geol. Landesamt Baden-Württemberg Jahresh., v. 3, p. 17-51 (1958).

Am. Mineralogist, v. 45, p. 254 (1960).

See also Uranospinite, p. 46.

\section{METAZELLERITE}

$\mathrm{Ca}\left(\mathrm{UO}_{2}\right)\left(\mathrm{CO}_{3}\right)_{2} \cdot 3 \mathrm{H}_{2} \mathrm{O}$

$\mathrm{U}=49.2$ percent.

Color: Yellow. A secondary mineral.

Am. Mineralogist v. 51, p. 1567-1578 (1966).

METAZEUNERITE

$\mathrm{Cu}\left(\mathrm{UO}_{2}\right)_{2}\left(\mathrm{AsO}_{4}\right)_{2} \cdot 8 \mathrm{H}_{2} \mathrm{O}$

$\mathrm{U}=46.4$ percent.

Color: Green. A common secondary mineral.

Dana VII, v. 2, p. 993-994.

Am. Mineralogist, v. 36, p. 249-255 (1951).

\section{MICROLITE}

$A_{2} B_{2} \mathrm{O}_{6}(\mathrm{O} \mathrm{OH}, \mathrm{F})$, where $A=\mathrm{Na}, \mathrm{Ca}, \mathrm{Fe}^{+2}, \mathrm{U}^{+4}, \mathrm{Sb}^{+3}, \mathrm{~Pb}, \mathrm{Th}, \mathrm{Zr}, \mathrm{Ce}$, $\mathrm{Y} ; B=\mathrm{Nb}, \mathrm{Ta}, \mathrm{Ti}, \mathrm{Sn}, \mathrm{Fe}^{+3}$.

In pyrochlore-microlite series, with $\mathrm{Ta}$ greater than $\mathrm{Nb}$.

$\mathrm{U}=\mathrm{up}$ to 10.4 percent; $\mathrm{Th}=0.2$ percent.

Color: Pale yellow, amber to dark brown. In granitic pegmatites and in pegmatites related alkalic igneous rocks.

Dana VII, v. 1, p. 748-754.

U.S. Geol. Survey Bull. 1064, p. 326-333 (1958).

Bismutomicrolite

Variety of microlite.

Am. Mineralogist, v. 43, p. 1223 (1958).

Calciotantalite

Possibly a mixture of microlite and tantalite.

Dana VII, v. 1, p. 787.

Djalmaite

Synonym of microlite.

Acad. Brasileira Ciênc. Anais, v. 22, p. 139-140 (1950).

Haddamite

Synonym of microlite.

Dana VII, v. 1, p. 748.

Metasimpsonite

An alteration product of simpsonite; later identified with microlite.

Dana VII, v1. 1, p. 748, 755 . 
MICROLITE-Continued

Neotantalite

An altered microlite with composition close to tantalite.

Dana VII, v. 1, p. 748, 750-751, 753.

Niobtantalpyrochlore

Synonym of microlite.

Chemie der Erde, v. 7, p. 56 (1932).

Tantalpyrochlore

Synonym of microlite.

Chemie der Erde, v. 7, p. 56 (1932).

MOCTEZUMITE

$\mathrm{PbO} \cdot \mathrm{UO}_{3} \cdot 2 \mathrm{TeO}_{2}$

$\mathrm{U}=\mathbf{2 4 . 2}$ percent.

Color: Bright orange. A rare secondary mineral.

Am. Mineralogist, v. 50, p. 1158-1163 (1965).

MOLURANITE

$\mathrm{UO}_{2} \cdot 3 \mathrm{UO}_{3} \cdot 7 \mathrm{MoO}_{3} \cdot 20 \mathrm{H}_{2} \mathrm{O}$

$\mathrm{U}=36$ percent.

Color: Black. Occurs in fissures in granulated albitite.

Am. Mineralogist, v. 43, p. 380 (1958); v. 45, p. 258 (1960).

MONAZITE

(Ce, La, Nd) $\mathrm{PO}_{4}$, with Th substituting for (Ce,La) and Si for $\mathrm{P}$.

$\mathrm{Th}$ is normally from a few percent to $\mathbf{1 0 . 6}$ percent, but series probably extends to 26.4 percent. $U$ is usually less than 0.1 percent.

Color: Yellow, brown, reddish brown. Widely disseminated as an accessory mineral in granites, gneisses, and pegmatites. In placer deposits. Rarely in veins.

Dana VII, v. 2, p. 691-696.

U.S. Geol. Survey Bull. 1064, p. 150-160 (1958).

Compare Cerphosphorhuttonite and cheralite, p. 14, 15.

Cryptolite

Synonym of monazite.

Dana VII, v. 2, p. 691.

Edwardsite

Synonym of monazite.

Dana VII, v. 2, p. 691.

Eremite

Synonym of monazite.

Dana VII, v. 2, p. 691.

Guadarramite

An intergrowth of radioactive monazite and some ilmenite.

Am. Mineralogist, v. 37, p. 1061 (1952).

Korarfveite

Impure monazite.

Dana VII, v. 2, p. 691.

Mengite

Synonym of monazite.

Dana VII, v. 2, p. 691. 
MONAZITE-Continued

Monazitoid

Synonym of monazite.

Dana VII, v. 2, p. 691.

Phosphocerite

Synonym of monazite.

Dana VII, v. 2, p. 691.

Turnerite

Synonym of monazite.

Dana VII, v. 2, p. 691, 695.

Urdite

Synonym of monazite.

Dana VII, v. 2, p. 691.

MOURITE

Hydrous uranous uranic molybdate.

$\mathrm{U}=\mathbf{1 8 . 5}$ percent.

Color: Violet. A rare secondary mineral.

Am. Mineralogist, v. 47, p. 1217 (1962).

NATROAUTUNITE (or sodium autunite, or soda-autunite)

$\mathrm{Na}_{2}\left(\mathrm{UO}_{2}\right)_{2}\left(\mathrm{PO}_{4}\right)_{2} \cdot 8 \mathrm{H}_{2} \mathrm{O}$

A member of the meta-autunite group.

$\mathrm{U}=46.7$ percent.

Color: Yellow. From granodiorite massifs.

The geology of uranium, 1958, p. 66-69. Tran.lated from Russian by Consultants Bur., Inc.

Am. Mineralogist, v. 43, p. 383 (1958).

NENADKEVITE

$\left(\mathrm{U}^{+4}, \mathrm{Y}, \mathrm{Ce}\right) \mathrm{U}^{+6}(\mathrm{Ca}, \mathrm{Mg}, \mathrm{Pb})\left(\mathrm{SiO}_{4}\right)_{2}(\mathrm{OH})_{4} \cdot n \mathrm{H}_{2} \mathrm{O}$

Much like coffinite, but perhaps distinct from it. Probably a high Umember in series thorite-uranothorite-nenadkevite (not to be confused with nenadkevichite).

$\mathrm{U}=19.2$ to 55.0 percent; $\mathrm{Th}=0.009$ to 0.3 percent.

Color: Black, greenish black, brown to reddish brown, orange and yellow. Occurs in zone of sodium metasomatism of iron-uranium deposits.

The geology of uranium, 1958, p. 43-55. Translated from Russian by Consultants Bur., Inc.

Am. Mineralogist, v. 42, p. 441-442 (1957).

NINGYOITE

$\left(\mathrm{U}^{+4}, \mathrm{Ca}, \mathrm{R} . \mathrm{E} .{ }^{*}\right)_{2}\left(\mathrm{PO}_{4}\right)_{2} \cdot 1-2 \mathrm{H}_{2} \mathrm{O}$.

Has a structure similar to that of rhabdophane.

$\mathrm{U}=20.4$ percent.

Color: Brownish green to brown. Occurs as coatings or filling cavities in $\mathrm{U}$ ore.

Am. Mineralogist, v. 44, p. 633-650 (1959).

* Rare earths.

268-008-67-5 
NOHLITE

$\left(\mathrm{Ca}, \mathrm{Mg}, \mathrm{Fe}^{+2}, \mathrm{Y}, \mathrm{U}\right)_{2}\left(\mathrm{Nb}, \mathrm{Zr}, \mathrm{Fe}^{+3}\right)_{3} \mathrm{O}_{10}$

Validity of species is doubtful.

$\mathrm{U}=13.0$ percent.

Color: Brownish black. From a feldspar quarry.

Dana VII, v. 1, p. 772-773.

NOVACEKITE

$\mathrm{Mg}\left(\mathrm{UO}_{2}\right)_{2}\left(\mathrm{AsO}_{4}\right)_{2} \cdot 8-10 \mathrm{H}_{2} \mathrm{O}$

Arsenate end-member of saléeite-novacekite series.

$\mathrm{U}=51.6$ to 53.7 percent.

Color: Yellow. A rare secondary mineral.

Am. Mineralogist, v. 36, p. 680-686 (1951).

Novacekite I and II

Various hydrates of novacekite.

Soc. Française Minéralogie et Cristallographie Bull., v. 81, p. 71 (1958).

\section{OBR UCHEVITE}

$(\mathrm{Y}, \mathrm{Na}, \mathrm{Ca}, \mathrm{U})(\mathrm{Nb}, \mathrm{Ta}, \mathrm{Ti}, \mathrm{Fe})_{2}(\mathrm{O}, \mathrm{OH})_{7} \cdot \mathrm{H}_{2} \mathrm{O}$

A member of the pyrochlore group with $\mathrm{Y}$ predominant.

$\mathrm{U}=8.1$ to 10.9 percent; $\mathrm{Th}=0.1$ to 0.2 percent.

Color: Brown to chocolate brown. From granitic pegmatites.

Am. Mineralogist, v. 43, p. 797 (1958).

Yttrobetafite

A uranoan variety of obruchevite.

$\mathrm{U}=10.9$ percent; $\mathrm{Th}=1.1$ percent.

Color: Greenish black.

Am. Mineralogist, v. 49, p. 440-441 (1964).

\section{ORLITE}

$3 \mathrm{PbO} \cdot 3 \mathrm{UO}_{3} \cdot 4 \mathrm{SiO}_{2} \cdot 6 \mathrm{H}_{2} \mathrm{O}$

Close to kasolite. Needs further study.

$\mathrm{U}=38.1$ percent.

Color: Light cream to yellow. A secondary mineral.

Am. Mineralogist, v. 43, p. 381 (1958).

\section{ORTHOCHEVKINITE}

Complex alumino-silicate of $\mathrm{Ti}, \mathrm{Fe}, \mathrm{Mg}, \mathrm{Ca}, \mathrm{Th}, \mathrm{Ce}$, and rare earths.

Polymorph of chevkinite.

Am. Mineralogist, v. 44, p. 115-137 (1959).

\section{PARAPITCHBLENDE}

Oxide of uranium, chiefly $\mathrm{U}^{+6}$, minor $\mathrm{U}^{+4}$.

Name given provisionally to material requiring further study. Probably a gummite-type alteration product of uraninite.

Am. Mineralogist, v. 43, p. 792-793 (1958).

\section{PARSONSITE}

$\mathrm{Pb}_{2}\left(\mathrm{UO}_{2}\right)\left(\mathrm{PO}_{4}\right)_{2}$

$\mathrm{U}=24.7$ to 28.8 percent.

Color: Yellow. A rare secondary mineral.

Dana VII, v. 2, p. 913-914.

Am. Mineralogist, v. 35, p. 245-250 (1950). 


\section{PAULITE}

Perhaps the arsenate analog of sabugalite.

$\mathrm{HAl}\left(\mathrm{UO}_{2}\right)_{4}\left(\mathrm{AsO}_{4}\right)_{4} \cdot 16 \mathrm{H}_{2} \mathrm{O}$ (No analysis.)

Color: Light yellow. A secondary mineral.

Am. Mineralogist, v. 46, p. 465 (1961).

\section{PERRIERITE}

$(\mathrm{Ce}, \mathrm{Ca}, \mathrm{Y}, \mathrm{Th})_{2}(\mathrm{Ti}, \mathrm{Fe}, \mathrm{Mg})_{2}(\mathrm{Si}, \mathrm{Al})_{2} \mathrm{O}_{11}(?)$

Perhaps a polymorph of chevkinite.

$\mathrm{Th}=3.6$ percent.

Am. Mineralogist, v. 36, p. 926 (1951); v. 44, p. 115-137 (1959).

\section{PHOSPHURANYLITE}

$\mathrm{Ca}\left(\mathrm{UO}_{2}\right)_{4}\left(\mathrm{PO}_{4}\right)_{2} \cdot(\mathrm{OH})_{4} \cdot 7 \mathrm{H}_{2} \mathrm{O}$

Isostructural with renardite and dewindtite.

$\mathrm{U}=63.3$ percent.

Color: Deep to golden yeliow. A secondary mineral.

Am. Mineralogist, v. 35, p. 756-763 (1950); v. 39, p. 444-451 (1954).

\section{PISEKITE}

An ill-defined minerai, essentially a niobate-tantalate-titanate of $U$ and rare earths, with Th and Sn. May be related to ampangabeite.

Color: Yellowish brown to black. From granite pegmatite.

Narodni Mus., Prague, Casopis Mineralogie Geologie, v. 1, p. 2 (1923).

Dana VII, v.1, p. 807-808.

\section{POLYCRASE}

$(\mathrm{Y}, \mathrm{Ca}, \mathrm{Ce}, \mathrm{U}, \mathrm{Th})(\mathrm{Ti}, \mathrm{Nb}, \mathrm{Ta})_{2} \mathrm{O}_{6}$

Part of the euxenite-polycrase series.

$\mathrm{U}=5.5$ to 12.4 percent; $\mathrm{Th}=$ up to 4.7 percent.

Color: Black. From granite pegmatites.

Dana VII, v. 1, p. 787-791.

Mineralog. Mag., v. 31, p. 763-780 (1958).

\section{PRIORITE}

$\left(\mathrm{Y}, \mathrm{Er}, \mathrm{Ca}, \mathrm{Fe}^{+2}, \mathrm{Th}\right)(\mathrm{Ti}, \mathrm{Nb})_{2} \mathrm{O}_{6}$

Part of the aeschynite-priorite series.

$\mathrm{U}=0.4$ to 3.4 percent; $\mathrm{Th}=0.5$ to 14.9 percent.

Color: Black. From granite pegmatites and nepheline syenites.

Dana VII, v. 1, p. 793-796.

Mineralog. Mag., v. 31, p. 763-780 (1958).

Blomstrandine (or blomstrandinite)

Synonym of priorite.

Dana VII, v. 1, p. 793-796.

PRZHEVALSKITE (or prjevalskite)

$\mathrm{Pb}\left(\mathrm{UO}_{2}\right)_{2}\left(\mathrm{PO}_{4}\right)_{2} \cdot 2 \mathrm{H}_{2} \mathrm{O}$

$\mathrm{U}=48.9$ percent.

Color: Bright greenish yellow. A rare secondary mineral.

Am. Mineralogist, v. 43, p. 381-382 (1958).

PSEUDO-AUTUNITE

$\left(\mathrm{H}_{3} \mathrm{O}\right)_{2} \mathrm{Ca}\left(\mathrm{UO}_{2}\right)\left(\mathrm{PO}_{4}\right)_{2} \cdot 2.5 \mathrm{H}_{2} \mathrm{O}$

Chemical and X-ray results indicate that the mineral is not a member of the autunite or meta-autunite group.

$\mathrm{U}=\mathbf{4 4 . 2}$ percent.

Color: Pale yellow to white. A secondary mineral.

Am. Mineralogist, v. 50, p. 1505-1506 (1965). 


\section{PYROCHLORE}

$A_{2} B_{2} \mathrm{O}_{6}(\mathrm{O}, \mathrm{OH}, \mathrm{F})$, where $A=\mathrm{Na}$, Ca mainly, also $\mathrm{Fe}^{+2}, \mathrm{U}^{+4}, \mathrm{Sb}^{+3}, \mathrm{~Pb}$, $\mathrm{Th}, \mathrm{Ce}, \mathrm{Y} ; B=\mathrm{Nb}$, Ta, Ti mainly, also $\mathrm{Sn}, \mathrm{Fe}^{+3}, \mathrm{~W}$.

In pyrochlore-microlite series, with $\mathrm{Nb}$ greater than $\mathrm{Ta}$.

$\mathrm{U}=$ up to 1.4 percent, usually, but as much as 17.1 percent in some varieties; $\mathrm{Th}=$ up to 5.5 percent.

Color: Pale yellow, amber to dark brown. In pegmatites derived from alkalic igneous rocks.

Dana VII, v. 1, p. 748-754.

U.S. Geol. Survey Bull. 1064, p. 326-333 (1958).

See also Betafite, p. 12; microlite, p. 27; obruchevite, p. 30; pandaite, p. 56; and wiikite, p. 48.

Azor-pyrrhit

Synonym of pyrochlore.

Naturw. Ver. Preuss. Rheinlande u. Westfalens Sitzungsber., v. 43, p. 217 (1886).

Chalcolamprite

Synonym of pyrochlore.

Dana VII, v. 1 , p. $748,750,754$.

Ellsworthite

An altered uranoan variety of pyrochlore related to hatchettolite.

Dana VII, v. 1, p. 748, 750-752, 755 .

Endeiolite

Similar in composition to chalcolamprite.

An altered pyrochlore(?).

Dana VII, v. 1,jp. 748, 754 .

Fluochlore

Synonym of pyrochlore(?).

Jour. Prakt. Chemie, v. 50, p. 186-187 (1850).

Hatchettolite

Uranoan variety of pyrochlore.

Dana VII, v. 1, p. 748, 750-752, 754.

Hydrochlore

Synonym of pyrochlore.

Jour. Prakt. Chemie, v. 50, p. 186-187 (1850).

Koppite

Synonym of pyrochlore.

Dana VII, v. 1 , p. 748,754 .

Marignacite

Synonym of pyrochlore.

Dana VII, v. 1, p. 748, 750-752, 755.

Niobpyrochlore

Synonym of pyrochlore.

Chemie der Erde, v. 7, p. 56 (1932).

Priazovite

Probably a variety of pyrochlore.

Am. Mineralogist, v. 50, p. 268 (1965). 


\section{PYROCHLORE-Continued}

\section{Pyrrhite}

Synonym of pyrochlore.

Dana VII, v. 1, p. 748, 752, 754.

Uranpyrochlore

Synonym of pyrochlore.

Uppsala Univ. Geol. Inst. Bull. 3, p. 181 (1896).

\section{RABBITTITE}

$\mathrm{Ca}_{3} \mathrm{Mg}_{3}\left(\mathrm{UO}_{2}\right)_{2}\left(\mathrm{CO}_{3}\right)_{6}(\mathrm{OH})_{4} \cdot 18 \mathrm{H}_{2} \mathrm{O}$

$\mathrm{U}=31.1$ percent.

Color: Pale green. A rare secondary mineral.

Am. Mineralogist, v. 40, p. 201-206 (1955).

\section{RANQUILITE}

$3 \mathrm{CaO} \cdot 4 \mathrm{UO}_{3} \cdot 10 \mathrm{SiO}_{2} \cdot 24 \mathrm{H}_{2} \mathrm{O}(?)$

Doubtful, needs further study.

$\mathrm{U}=39.7$ percent.

Occurs in fissures in gypsum

Am. Mineralogist, v. 45, p. 1078-1086 (1960).

\section{RAUVITE}

$\mathrm{CaO} \cdot 2 \mathrm{UO}_{3} \cdot 5 \mathrm{~V}_{2} \mathrm{O}_{5} \cdot 16 \mathrm{H}_{2} \mathrm{O}(?)$

$\mathrm{U}=26.1$ percent.

Color: Purplish to bluish black. A secondary mineral.

Dana VII, v. 2, p. 1058.

U.S. Geol. Survey Bull. 1009-B, p. 13-62 (1954).

\section{RENARDITE}

$\mathrm{Pb}\left(\mathrm{UO}_{2}\right)_{4}\left(\mathrm{PO}_{4}\right)_{2}(\mathrm{OH})_{4} \cdot 7 \mathrm{H}_{2} \mathrm{O}$

Isostructural with phosphuranylite and dewindtite.

$\mathrm{U}=57.3$ percent.

Color: Yellow. A rare secondary mineral.

Dana VII, v. 2, p. 928.

Am. Mineralogist, v. 39, p. 444-451 (1954).

\section{RICHETITE}

Contains $\mathrm{Pb}$ and $\mathrm{U}$.

Color: Black. A rare secondary mineral.

Soc. Belge Géologie, Paléontologie, et Hydrologie Bull., v. 70, p. 212-225 (1947).

Soc. Française Minéralogie et Cristallographie Bull., v. 82, p. 264-265 (1959).

Am. Mineralogist, v. 33, p. 384 (1948).

\section{R UTHERFORDINE}

$\left(\mathrm{UO}_{2}\right) \mathrm{CO}_{3}$

$\mathrm{U}=72.1$ percent.

Color: Yellow. A rare secondary mineral.

Dana VII, v. 2, p. 274-275.

Am. Mineralogist, v. 41, p. 127-122; p. 844-850 (1956).

\section{Diderichite}

Synonym of rutherfordine.

Am. Mineralogist, v. 41, p. 127-133 (1956). 


\section{SABUGALITE}

$\mathrm{HAl}\left(\mathrm{UO}_{2}\right)_{4}\left(\mathrm{PO}_{4}\right)_{4} \cdot 16 \mathrm{H}_{2} \mathrm{O}$

$\mathrm{U}=53.6$ percent.

Color: Yellow. A rare secondary mineral.

Am. Mineralogist, v. 36, p. 671-679 (1951).

\section{SALEEITE}

$\mathrm{Mg}\left(\mathrm{UO}_{2}\right)_{2}\left(\mathrm{PO}_{4}\right)_{2} \cdot 8-10 \mathrm{H}_{2} \mathrm{O}$

Phosphate end-member of the saléeite-novacekite series. $\mathrm{U}=50.9$ to 53.0 percent.

Color: Yellow. A rare secondary mineral.

Dana VII, v. 2, p. 988-989.

Am. Mineralogist, v. 36, p. 680-686 (1951).

Magnesium-phosphoruranit

Synonym of saléeite.

Mineralog. Mag., v. 28, p. 732 (1949).

\section{SAMARSKITE}

( Y, Ce, U, Ca, Fe, $\mathrm{Pb}, \mathrm{Th})(\mathrm{Nb}, \mathrm{Te}, \mathrm{Ti}, \mathrm{Sn})_{2} \mathrm{O}_{6}$

$\mathrm{U}=8.4$ to 16.6 percent; $\mathrm{Th}=\mathrm{up}$ to 3.7 percent.

Color: Velvet black to brown. From granite pegmatites.

Dana VII, v. 1, p. 797-800.

Mineralog. Mag., v. 31, p. 763-780 (1958).

Ampangabeite

Synonym of samarskite.

Soc. Française Minéralogie et Cristallographie Bull., v. 83, p. 295-309 (1960).

Am. Mineralogist, v. 46, p. 770 (1961).

Annerödite

Samarskite with parallel overgrowths of columbite.

Dana VII, v. 1, p. 797, 799 .

Eytlandite

Synonym of samarskite.

Dana VII, v. 1, p. 797.

Hydroeuxenite (or ampangabeite)

Dana VII, v. 1, p. 806.

Hydrosamarskite

An altered samarskite.

Dana VII, v. 1, p. 799-800.

Nuevite

Synonym of samarskite.

Am. Mineralogist, v. 36 , p. 358 (1951).

Plumboniobite

A niobate of $\mathrm{Y}, \mathrm{U}, \mathrm{Pb}, \mathrm{Fe}$, and rare earths.

May be a plumbian variety of samarskite.

Dana VII, v. 1, p. 800.

Rogersite

Probably an altered samarskite.

Of doubtful validity.

Dana VII, v. 1, p. 800 . 


\section{SAMARSKITE-Continued}

Toddite

A mixture of samarskite and columbite.

Am. Mineralogist, v. 47, p. 1363-1379 (1962).

Uranotantalite

Synonym of samarskite.

Dana VII, v. 1, p. 797.

Vietinghofite

Supposedly a ferroan samarskite.

Dana VII, v. 1, p. $800-801$.

Yttro-ilmenite

Synonym of samarskite.

Dana VII, v. 1, p. 797.

SARYARKITE

$(\mathrm{Ca}, \mathrm{Y}, \mathrm{Th})_{2} \mathrm{Al}_{4}\left(\mathrm{SiO}_{4}, \mathrm{PO}_{4}\right)_{4}(\mathrm{OH}) \cdot 9 \mathrm{H}_{2} \mathrm{O}$

Apparently a member of the rhabdophane group.

$\mathrm{Th}=6.8$ to 8.0 percent.

Color: White. Occurs in propylitized acid effusives and in altered granitic rocks.

Am. Mineralogist, v. 49, p. 1775-1776 (1964).

SCHOEPITE (or schoepite I)

$\mathrm{UO}_{3} \cdot 2 \mathrm{H}_{2} \mathrm{O}$

$\mathrm{U}=71.9$ percent.

Color: Yellow. A secondary mineral.

Dana VII, v. 1, p. 627-628.

U.S. Geol. Survey Bull. 1064, p. 72-78 (1958).

Soc. Française Minéralogie et Cristallographie Bull., v. 82, p. 242-244 (1959).

Am. Minéralogist, v. 45, p. 1026-1061 (1960); v. 50, p. 235-239 (1965).

Epiianthinite

Yellow pseudomorphous alteration of ianthinite; identical with schoepite.

Am. Mineralogist, v. 32, p. 344-350 (1947); v. 44, p. 1104 (1959).

Metaschoepite (or schoepite II)

Closely related to schoepite; contains less $\mathrm{H}_{2} \mathrm{O}$.

Am. Mineralogist, v. 45, p. 1026-1061 (1960); v. 50, p. 235-239 (1965).

Paraschoepite (or schoepite III)

Closely related to schoepite; contains less $\mathrm{H}_{2} \mathrm{O}$.

Am. Mineralogist, v. 32, p. 344-350 (1947); v. 33, p. 513-514 (1948); v. 45 , p. $1026-1061$ (1960); v. 50 , p. 235-239 (1965).

U.S. Geol. Survey Bull. 1064, p. 72-78 (1958).

\section{SCHROECKINGERITE}

$\mathrm{NaCa}_{3}\left(\mathrm{UO}_{2}\right)\left(\mathrm{CO}_{3}\right)_{3}\left(\mathrm{SO}_{4}\right) \mathrm{F} \cdot 10 \mathrm{H}_{2} \mathrm{O}$

$\mathrm{U}=26.8$ percent.

Color: Greenish yellow. A secondary mineral.

Dana VII, v. 2, p. 236.

Am. Mineralogist, v. 39, p. 901-907 (1954).

Dakeite

Synonym of schroeckingerite.

Dana VII, v. 2, p. 236. 


\section{SCHROECKINGERITE-Continued}

Neogastunite

Synonym of schroeckingerite.

Mineralog. petrog. Mitt., v. 2, p. 311-312 (1951).

\section{SEDOVITE}

$\mathrm{U}\left(\mathrm{MoO}_{4}\right)_{2}(?)$

$\mathrm{U}=36$ percent.

Color: Brown to reddish brown. A secondary mineral from the oxidation zone.

Am. Mineralogist, v. 51, p. 530 (1966).

\section{SENGIERITE}

$\mathrm{Cu}\left(\mathrm{UO}_{2}\right)_{2}\left(\mathrm{VO}_{4}\right)_{2} \cdot 8-10 \mathrm{H}_{2} \mathrm{O}$

$\mathrm{U}=47.0$ to 48.7 percent.

Color: Yellowish green. A rare secondary mineral.

Dana VII, v. 2, p. 1047.

U.S. Geol. Survey Bull. 1064, p. 258-260 (1958).

\section{SHARPITE}

Perhaps $\left(\mathrm{UO}_{2}\right)\left(\mathrm{CO}_{3}\right) \cdot \mathrm{H}_{2} \mathrm{O}$ or $6 \mathrm{UO}_{3} \cdot 5 \mathrm{CO}_{2} \cdot 7 \mathrm{H}_{2} \mathrm{O}$

Composition needs to be checked.

$\mathrm{U}=68.4$ to 68.6 percent.

Color: Greenish yellow. A secondary mineral.

Dana VII, v. 2, p. 275.

\section{SINICITE}

Perhaps $A B_{2}(\mathrm{O}, \mathrm{OH})_{7}$, where $A=\mathrm{Ce}, \mathrm{Y}, \mathrm{U}, \mathrm{Th} ; B=\mathrm{Ti}$, Nb mainly.

$\mathrm{U}=5.0$ to 5.7 percent; $\mathrm{Th}=7.0$ to 7.3 percent.

Needs further study; may be a variety of aeschynite.

Color: Blackish brown to reddish brown. Occurs in granite pegmatite.

Am. Mineralogist, v. 44, p. 467 (1959).

SKLODOWSKITE (or sklodovskite)

$\mathrm{Mg}\left(\mathrm{UO}_{2}\right)_{2}\left(\mathrm{SiO}_{3}\right)_{2}(\mathrm{OH})_{2} \cdot 5 \mathrm{H}_{2} \mathrm{O}$

Isostructural with uranophane and cuprosklodowskite.

$\mathrm{U}=56.6$ percent.

Color: Yellow. A rare secondary mineral.

U.S. Geol. Survey Bull. 1064, p. 300-304 (1958).

Chinkolobwite (or shinkolobwite)

Synonym of sklodowskite.

Mineralog. Abs., v. 3, p. 115 (1926).

SODD YITE (or soddite)

$\left(\mathrm{UO}_{2}\right)_{5}\left(\mathrm{SiO}_{4}\right)_{2}(\mathrm{OH})_{2} \cdot 5 \mathrm{H}_{2} \mathrm{O}$

$\mathrm{U}=71.8$ percent.

Color: Yellow. A secondary mineral.

Mineralog. Abs., v. 3, p. 371 (1927).

U.S. Geol. Survey Bull. 1064, p. 312-315 (1958).

\section{SODIUM URANOSPINITE}

$\left(\mathrm{Na}_{2}, \mathrm{Ca}\right)\left(\mathrm{UO}_{2}\right)_{2}\left[(\mathrm{As}, \mathrm{P}) \mathrm{O}_{4}\right]_{2} \cdot 5 \mathrm{H}_{2} \mathrm{O}$

$\mathrm{A}$ member of the meta-autunite group.

$\mathrm{U}=48.5$ percent.

Color: Yellow green to lemon and straw yellow.

A secondary mineral in a primary hydrothermal deposit.

Am. Mineralogist, v. 43, p. 383-384 (1958). 


\section{SODIUM URANOSPINITE-Continued}

\section{Ellweilerite}

Probably a Ca-free variety of sodium uranospinite.

Am. Mineralogist, v. 46, p. 465 (1961).

\section{STEENSTR UPINE}

$(\mathrm{Ca}, \mathrm{La}, \mathrm{Th}, \mathrm{Ca}, \mathrm{Na})_{2}(\mathrm{Mn}, \mathrm{Fe})\left(\mathrm{SiO}_{3}\right)_{2}(\mathrm{OH})_{2} \cdot 2 \mathrm{H}_{2} \mathrm{O}$

$\mathrm{Th}=1.9$ to 9.96 percent; $\mathrm{U}=$ up to 0.18 percent.

Color: Brown to black. From syenite and alkalic rock pegmatites.

Dana VI, p. 415.

Vlasov, K. A., Kuz'menko, M. V., and Es'kova, E. M., The Lovozero alkali massif, 1959, p. 418-422, Moscow, Akad. Nauk SSSR, Izdatel. (in Russian).

Manganosteenstrupine

A variety of steenstrupine with high Mn content.

Am. Mineralogist, v. 45, p. 1132 (1960).

Compare Thorosteenstrupine, p. 40.

\section{STUDTITE}

Hydrated carbonate of $\mathrm{U}$ and $\mathrm{Pb}$.

Species not well defined.

Color: Yellow. A secondary mineral.

Dana VII, v. 2, p. 275.

SWARTZITE

$\mathrm{CaMg}\left(\mathrm{UO}_{2}\right)\left(\mathrm{CO}_{3}\right)_{3} \cdot 12 \mathrm{H}_{2} \mathrm{O}$

$\mathrm{U}=32.6$ percent.

Color: Green. A rare secondary mineral.

Dana VII, v. 2, p. 238-239.

THORBASTNAESITE

$\mathrm{Th}(\mathrm{Ce}, \mathrm{Ca})\left(\mathrm{CO}_{3}\right)_{2} \mathrm{~F}_{2} \cdot 3 \mathrm{H}_{2} \mathrm{O}$

$\mathrm{Th}=41.0$ percent; $\mathrm{U}=0.09$ percent.

Color: Brown. Accessory mineral in iron-rich albitites; selvages of veinlets and stockworks.

Am. Mineralogist, v. 50, p. 1505 (1965).

Compare Bastnaesite, p. 52.

\section{THORIANITE}

Includes alpha-, beta-, and gamma-thorianite.

$(\mathrm{Th}, \mathrm{U}) \mathrm{O}_{2}$

Forms complete series with uraninite.

Division between thorianite and uraninite at $\mathrm{Th}: \mathrm{U}=1: 1$ atomic ratio $\mathrm{U}=\mathrm{up}$ to 44.6 percent; $\mathrm{Th}=43.4$ to 87.9 percent.

Color: Gray, black, brownish black. From placers.

Dana VII, v. 1, p. 620-622.

U.S. Geol. Survey Bull. 1064, p. 47-53 (1958).

\section{Aldanite}

A uranoan plumboan thorianite.

$\mathrm{U}=12.4$ to 16.7 percent, $\mathrm{Th}=56.5$ to 60.9 percent.

Soviet Geol., 1941, no. 6, p. 105-107.

Am. Mineralogist, v. 40, p. 369 (1955).

Uranothorianite

Synonym for thorianite containing U.

Am. Jour. Sci., v. 26, p. 47 (1933). 


\section{THORITE}

$\mathrm{ThSiO}_{4}$

$\mathrm{U}=$ up to 10.1 percent; $\mathrm{Th}=25.2$ to 64.1 percent.

Color: Yellow, brown, black. From nepheline syenites and granites.

Dana VI, p. $480-490$.

Am. Mineralogist, v. 38, p. 1007-1018 (1953).

Canadian Mineralogist, v. 6, p. 1-4 (1957).

U.S. Geol. Survey Bull. 1064, p. 265-276 (1958).

See also Varieties listed under thorogummite, p. 39; hydrocerite, p. 21.

Auerlite

Phosphatian variety of thorite or thorogrummite with $\left(\mathrm{PO}_{4}\right):\left(\mathrm{SiO}_{4}\right) \approx$ 0.8: 1 .

Am Mineralogist, v. 38, p. 1007-1018 (1953).

U.S. Geol. Survey Bull. 1064, p. 267-277 (1958).

Calciothorite

May be calcian variety of thorite or thorogummite.

Am. Mineralogist, v. 38, p. 1007-1018 (1953).

U.S. Geol. Survey Bull. 1064, p. 277 (1958).

\section{Enalite}

Uranoan variety of thorite or thorogummite.

Am. Mineralogist, v. 18, p. 223 (1933) ; v. 38, p. 1007-1018 (1953).

U.S. Geol. Survey Bull. 1064, p. 279 (1958).

Eucrasite

Possibly a variety of thorite or thorogummite high in rare earths.

Am. Mineralogist, v. 38, p. 1007-1018 (1953).

U.S. Geol. Survey Bull. 1064, p. 278-279 (1958).

Ferrothorite

An altered variety of thorite; high in Fe.

Am. Mineralogist, v. 14, p. 78 (1929); v. 38, p. 1007-1018 (1953).

U.S. Geol. Survey Bull. 1064, p. 279 (1958).

Freyalite

Variety of thorite high in rare earths.

Am. Mineralogist, v. 38, p. 1007-1018 (1953).

Jiningite

$A$ variety of thorite high in $\mathrm{Fe}_{2} \mathrm{O}_{3}$ (17.28 percent), $\mathrm{P}_{2} \mathrm{O}_{5}$ (6.08 percent) and $\mathrm{V}_{2} \mathrm{O}_{5}(0.97$ percent).

$\mathrm{Th}=39.4$ percent; $\mathrm{U}=0.39$ percent.

Am. Mineralogist, v. 45, p. 755 (1960).

Mozambikite

Possibly a variety of thorite or thorogummite.

$\mathrm{Th}=51.7$ percent; $\mathrm{U}=5.1$ percent.

Internat. Geol. Cong., 21st, Copenhagen, Rept., pt. 17, p. 53-62 (1960).

Am. Mineralogist, v. 45, p. 1317 (1960).

Orangite

Synonym of thorite.

Dana VI, p. 488-489.

Shentulite (or shen-t'u-shih)

A variety of thorite; high in $\mathrm{P}_{2} \mathrm{O}_{5}$ (5.16 percent) and $\mathrm{As}_{2} \mathrm{O}_{5}$ (3.62 percent). $\mathrm{Th}=46.4$ percent.

Am. Mineralogist, v. 45 , p. 755 (1960). 
THORITE-Continued

Silicosmirnovskite

Similar to smirnovksite, but with $\mathrm{Si}>\mathrm{P}$.

$\mathrm{Th}=39.8$ percent.

Probably thorite or thorogummite.

Am. Mineralogist, v. 47, p. 419-420 (1962).

Smirnovskite

Abbreviated theoretical formula: (Th, $\mathrm{Ca}, \mathrm{Ce})(\mathrm{P}, \mathrm{Si}, \mathrm{Al})(\mathrm{OH})(\mathrm{O}, \mathrm{OH}, \mathrm{F})_{4}$

Probably a phosphate-rich metamict thorite, near auerlite.

$\mathrm{U}=0.08$ percent; $\mathrm{Th}=\mathbf{4 5 . 0}$ to 46.7 percent.

Color: Reddish brown to dirty brown; also orange, yellow, or colorless.

Occurs in cassiterite-feldspar-quartz veins.

Am. Mineralogist, v. 43, p. 386-387 (1958).

Uranothorite

Uranoan variety of thorite.

Am. Mineralogist, v. 36, p. 557-562 (1951).

Wisaksonite

Uranoan variety of thorite.

Am. Mineralogist, v. 39, p. 825-829 (1954).

Yanshainshynite

Probably a phosphatian thorite.

$\mathrm{Th}=37.7$ percent.

Am. Mineralogist, v. 46, p. 1200-1201 (1961).

THOROGUMMITE

$\mathrm{Th}\left(\mathrm{SiO}_{4}\right)_{1-x}(\mathrm{OH})_{4 x}$

Isostructural with thorite.

$\mathrm{U}=2.5$ to 31.4 percent; $\mathrm{Th}=18.2$ to 50.8 percent.

Am. Mineralogist, v. 38, p. 1007-1018 (1953).

See also Varieties listed under thorite, p. 38-39.

Compare Coffinite, p. 15.

Chlorothorite

Synonym of thorogummite.

Am. Mineralogist, v. 38, p. 1007-1018 (1953).

Hyblite

Includes alpha- and beta-hyblite.

Synonym of thorogummite.

Am. Mineralogist, v. 38, p. 1007-1018 (1953).

U.S. Geol. Survey Bull. 1064, p. 280 (1958).

Hydrothorite

Synonym of thorogummite.

Am. Mineralogist, v. 38, p. 1007-1018 (1953).

Hydroxyl-thorite

Synonym of thorogummite.

U.S. Geol. Survey Bull. 1064, p. 280 (1958).

Mackintoshite

Synonym of thorogummite.

Am. Mineralogist, v. 38, p. 1007-1018 (1953). 


\section{THOROGUMMITE-Continued}

Maitlandite

$2(\mathrm{~Pb}, \mathrm{Ca}) \mathrm{O} \cdot 3 \mathrm{ThO}_{2} \cdot 8 \mathrm{SiO}_{2} \cdot 23 \mathrm{H}_{2} \mathrm{O}$

Synonym of thorogummite.

$\mathrm{U}=31.3$ percent; $\mathrm{Th}=21.7$ to 22.7 percent.

Am. Mineralogist, v. 38, p. 1007-1018 (1953).

Zapiski Vses. Mineralog. Obshch., v. 87, p. 667-674 (1958).

Nicolayite

$2(\mathrm{~Pb}, \mathrm{Ca}) \mathrm{O} \cdot 3 \mathrm{ThO}_{2} \cdot 4 \mathrm{UO}_{2} \cdot 8 \mathrm{SiO}_{2} \cdot 21 \mathrm{H}_{2} \mathrm{O}$

Synonym of thorogummite.

$\mathrm{U}=31.1$ percent; $\mathrm{Th}=21.5$ percent.

Am. Mineralogist, v. 38, p. 1007-1018 (1953).

Pilbarite

A mixture of thorogummite and kasolite.

Am. Mineralogist, v. 42, p. $908-910$ (1957).

THOROSTEENSTR UPINE

$(\mathrm{Ca}, \mathrm{Th}, \mathrm{Mn})_{3} \mathrm{Si}_{4}(\mathrm{O}, \mathrm{F})_{12} \cdot 5-7 \mathrm{H}_{2} \mathrm{O}$

$\mathrm{Th}=31.2$ percent.

Color: Dark brown, nearly black. Occurs in metasomatic veins.

Zapiski Vses. Mineralog. Obshch., v. 91, p. 325-330 (1962).

Am. Mineralogist, v. 48, p. 433-434 (1963).

THORUTITE (or torutite)

( $\mathrm{Th}, \mathrm{Ca}, \mathrm{U}) \mathrm{Ti}_{2}(\mathrm{O}, \mathrm{OH})_{8}$

Thorium analog of brannerite.

$\mathrm{U}=2.2$ percent; $\mathrm{Th}=47.5$ percent.

Color: Black. Occurs in syenite massif.

Am. Mineralogist, v. 43, p. 1007 (1958); v. 48, p. 1419-1420 (1963).

Smirnovite

Synonym of thorutite.

Am. Mineralogist, v. 43, p. 1007 (1958).

\section{THUCHOLITE}

A complex of uraninite with hydrocarbons.

$\mathrm{U}=$ up to 53 percent in the ash. $\mathrm{Th}=$ up to 48 percent in the ash.

Color: Black. Mainly from pegmatite dikes.

Great Britain Geol. Survey Bull., v. 3, p. 1-19 (1951).

Am. Mineralogist, v. 38, p. 802 (1953)

Econ. Geology, v. 55, p. 1716-1738 (1960).

Anthraxolite

A nickeliferous and uraniferous hydrocarbon.

$\mathrm{U}=\mathbf{0 . 0 0 3}$ percent.

Am. Mineralogist, v. 19, p. 426-428 (1934).

Heinrich, E. W., Mineralogy and geology of radioactive raw materials, 1958, p. 121.

Asphaltite (or asphaltum)

Includes solid bituminous hydrocarbonas knowns as albertite, impsonite, gilsonite, grahamite, and uintahite.

$\mathrm{U}=0.001$ percent.

Mineralog. Mag., v. 15, p. 417 (1910). 


\section{THUCHOLITE-Continued}

Broggite

A variety of asphaltite.

Mineralog. Mag., v. 24, p. 604 (1937).

Carbocer

A material similar to thucholite, high in Ce.

Great Britain Geol. Survey Bull., v. 3, p. 3 (1951).

Am. Mineralogist, v. 38, p. 804 (1953).

Carburan

A hydrocarbon complex related to thucholite.

$\mathrm{U}=4.3$ percent.

Mineralog. Abs., v. 6, p. 437 (1936).

Grahamite

A uraniferous hydrocarbon like thucholite.

Heinrich, E. W., Mineralogy and geology of radioactive raw materials, 1958, p. 121.

Sogrenite

An organo-uranium complex like thucholite.

$\mathrm{U}=9.8$ to $\mathbf{1 6 . 6}$ percent, from microchemical analysis of inorganic part.

Color: Black. In ankerite-calcite veins cutting Upper Precambrian limestone.

Am. Mineralogist, v. 43, p. 382 (1958).

Titanothucholite

Titanian variety of thucholite.

Mineralog. Abs., v. 9, p. 37 (1944).

TORBERNITE

$\mathrm{Cu}\left(\mathrm{UO}_{2}\right)_{2}\left(\mathrm{PO}_{4}\right)_{2} \cdot 12 \mathrm{H}_{2} \mathrm{O}$

$\mathrm{U}=47.1$ percent.

Color: Green. A common secondary mineral.

Dana VII, v. 2, p. 981-984.

See also Metatorbernite, p. 26.

Chalcolite (or chalkolith)

Synonym of torbernite.

Dana VII, v. 2, p. 981.

Copper uranite

Synonym of torbernite.

Dana VII, v. 2, p. 981.

Cuprouranit

Synonym of torbernite.

Dana VII, v. 2, p. 981.

Kupferphosphoruranit

Synonym of torbernite.

Mineralog. Mag., v. 28, p. 732 (1949).

Torberite

Synonym of torbernite.

Brooke, H. J., and Miller, W. H., Introduction to Mineralogy, p. 517 (1852). 


\author{
TORBERNITE-Continued \\ Uranite \\ Synonym of torbernite-autunite group. \\ Dana VII, v. 2, p. 981-984. \\ Uran-mica \\ Synonym of torbernite. \\ Jameson, Robert, System of Mineralogy (1820). \\ Uranphyllit \\ Synonym of torbernite. \\ Dana VII, v. 2, p. 981.
}

\title{
TRITOMITE
}

A borosilicate of $\mathrm{Ce}, \mathrm{Y}, \mathrm{Th}, \mathrm{Ca}$, and $\mathrm{F}$; exact formula uncertain.

$\mathrm{Th}=7.5$ to 8.3 percent.

Color: Dark brown. From nepheline syenite pegmatite.

Dana VI, p. 416.

TROEGERITE

Probably $\mathrm{H}_{2}\left(\mathrm{UO}_{2}\right)_{2}\left(\mathrm{AsO}_{4}\right)_{2} \cdot 8 \mathrm{H}_{2} \mathrm{O}$

New analysis is needed.

$\mathrm{U}=55.1$ percent.

Color: Lemon yellow. A rare secondary mineral.

Am. Mineralogist, v. 38, p. 1159-1168 (1953).

Dana VII, v. 2, p. 966-967.

TSCHEFFKINITE. See Chevkinite, p. 15.

\section{TYUYAMUNITE}

$\mathrm{Ca}\left(\mathrm{UO}_{2}\right)_{2}\left(\mathrm{VO}_{4}\right)_{2} \cdot 5-8 \mathrm{H}_{2} \mathrm{O}$

$\mathrm{U}=49.4$ to 54.1 percent.

Color: Yellow. A common secondary mineral.

Dana VII, v. 2, p. 1045-1047.

U.S. Geol. Survey Bull. 1064, p. 248-253 (1958).

See also Metatyuyamunite, p. 26.

Calciocarnotite

Synonym of tyuyamunite.

Dana VII, v. 2, P. 1045.

\section{UMOHOITE}

Close to $\left(\mathrm{UO}_{2}\right)\left(\mathrm{MoO}_{4}\right) \cdot 4 \mathrm{H}_{2} \mathrm{O}(?)$

$\mathrm{U}=47.4$ percent.

Color: Black to bluish black. A rare secondary mineral.

U.S. Atomic Energy Commission, RME-3046, p. 45, April, 1953.

Am. Mineralogist, v. 42, p. 657-660 (1957); v. 44, p. 920-925, 12481260 (1959).

\section{UNNAMED MINERALS}

(Antipov, 1900)

Essentially $\mathrm{Cu}\left(\mathrm{UO}_{2}\right)\left(\mathrm{CO}_{3}\right)_{2} \cdot 10 \mathrm{H}_{2} \mathrm{O}$

$\mathrm{U}=37.6$ percent.

Gesell. Mineralog. St. Petersburg Verh., v. 38, p. 38 (1900).

(Belova and others, 1963)

$\left(\mathrm{UO}_{2}\right)_{3}\left(\mathrm{PO}_{4}\right)_{2} \cdot 8 \mathrm{H}_{2} \mathrm{O}$

Phosphate analog of troegerite.

Voprosy Prikladnaya Radiogeol. Sbornik, 1963, p. 174-177.

Am. Mineralogist, v. 50, p. 265-266 (1965). 


\section{UNNAMED MINERALS-Continued}

(Chernik, 1922)

Ill-defined vanadate of $\mathrm{Cu}$ and $\mathrm{U}$.

Has same qualitative chemical composition as sengierite.

$\mathrm{U}=32.5$ percent.

Acad. Sci. URSS Bull., v. 16, p. 505-514 (1922).

(Chirvinsky, 1925)

An incompletely described mineral presumed to contain $U$ by virtue of its properties and association. May be a variety of metatyuyamunite.

Mineralog. Mag., v. 20, p. 287 (1925).

U.S. Geol. Survey Bull. 1064, p. 257 (1958).

(Frondel, 1956)

Mineral A

An oxide of $\mathrm{U}$, or a uranate, containing $\mathrm{Pb}, \mathrm{K}, \mathrm{Na}, \mathrm{Ca}$, and $\mathrm{Ba}$.

Color: Orange yellow or golden yellow. From pegmatites.

Am. Mineralogist, v. 41, p. 560 (1956).

Mineral B

Possibly the calcium analog of kasolite.

Color: Straw yellow to pale yellowish brown. From pegmatites.

Am. Mineralogist, v. 41, p. 560-561 (1956).

(Melkov, 1946)

Contains $\mathrm{Bi}, \mathrm{U}, \mathrm{PO}_{4}$, and $\mathrm{H}_{2} \mathrm{O} . \mathrm{Na}, \mathrm{K}$ in traces.

May be the phosphate analog of walpurgite.

Color: Yellow. A secondary mineral.

Am. Mineralogist, v. 43, p. 383 (1958).

(Protas, 1957)

$\mathrm{CaO} \cdot 6 \mathrm{UO}_{3} \cdot 11 \mathrm{H}_{2} \mathrm{O}$

Calcium analog of becquerelite.

$\mathrm{U}=74.2$ percent.

Color: Yellow. A secondary mineral.

Acad. Sci. [Paris] Comptes Rendus, v. 244,p. 91-93 (1957).

Soc. Française Minéralogie et Cristallographie Bull., v. 82, p. 246-249 (1959).

(Protas, 1959)

Contains U, Sr, $\mathrm{Ca}, \mathrm{H}_{2} \mathrm{O}$.

$\mathrm{U}=69.8$ percent.

Color: Reddish orange. A secondary mineral.

Soc. Française Minéralogie et Cristallographie Bull. v. 82, p. 250-252 (1959).

Dehydration of the above mineral gives a product with an $\mathrm{X}$-ray powder pattern identical to that of a pale mineral found in very small amounts with the above.

(Soboleva and Pudovkina, 1957)

$(\mathrm{Cu}, \mathrm{Ca})\left(\mathrm{UO}_{2}\right)_{2}[(\mathrm{P}, \mathrm{As})]_{\mathrm{g}} \cdot 18 \mathrm{H}_{2} \mathrm{O}(?)$

Uranium mica of mixed composition. Needs further study.

$\mathrm{U}=35.5$ to 40.0 percent.

Color: Green to yellow. A secondary mineral.

Mineraly urana, spravochnik, Moscow, 1957, p. 202. 


\section{UNNAMED MINERALS-Continued}

(Walenta, 1958)

Mineral D, Mineral E

Contains $\mathrm{U}$ and As.

Color: Citron yellow. Secondary minerals.

Am. Mineralogist, v. 41, p. 560-561 (1956).

Soc. Française Minéralogie et Cristallographie Bull., v. 81, p. 70 (1958).

Geol. Landesamt Baden-Wurttemberg Jahresh. v. 3, p. 17-51 (1958).

\section{URACONITE}

This name has been used for uranium sulfates, but lacks specific meaning and should be abandoned.

Dana VII, v. 2, p. 600.

\section{URAMPHITE}

$\left(\mathrm{NH}_{4}\right)\left(\mathrm{UO}_{2}\right)\left(\mathrm{PO}_{4}\right) \cdot 3 \mathrm{H}_{2} \mathrm{O}$

Possibly a member of the meta-autunite or meta-torbernite groups.

$\mathrm{U}=54.5$ percent.

Color: Bottle green to pale green. Occurs in oxidized zone of a uraniumcoal deposit.

The geology of uranium, 1958 , p. 56-60, translated from Russian by Consultants Bur., Inc.

Am. Mineralogist, v. 44, p. 464 (1959).

\section{URANINITE}

Ideally $\mathrm{UO}_{2}$, but better expressed as $\left(\mathrm{U}^{+4_{1-x}}, \mathrm{U}^{+6}{ }_{x}\right) \mathrm{O}_{2+x}$.

Usually somewhat oxidized and varying in composition to at least $\left(\mathrm{U}^{+4}, \mathrm{U}^{+6}\right) \mathrm{O}_{\mathbf{2 . 6}}$.

Also contains $\mathrm{Th}, \mathrm{Pb}, \mathrm{Y}$, and other rare earths in solid solution. Forms complete series with thorianite. Division between uraninite and thorianite at $\mathrm{U}: \mathrm{Th}=1: 1$.

$\mathrm{U}=46.5$ to 88.2 percent; $\mathrm{Th}=$ zero to 45.3 percent.

Color: Velvety brownish, grayish, and greenish black. In granite and syenite pegmatites (crystals); in hydrothermal sulfide veins (pitchblende); in sandstone-type deposits.

Dana VII, v. 1, p. 611-620.

Am. Mineralogist, v. 37, p. 363-385 (1952).

U.S. Geol. Survey Bull. 1064, p. 11-47 (1958).

See also Hydronasturan, p. 22; parapitehblende, p. 30.

Bröggerite

A thorian variety of uraninite.

Dana VII, v. 1, p. 611-614.

Cleveite

A variety of uraninite containing rare earths.

Dana VII, v. 1, p. 611-614.

Coracite

Synonym of uraninite.

Dana VII, v. 1, p. 611, 615, 617.

Nasturan

Synonym of pitchblende.

Dana VII, v. 1, p. 611, 614, 617. 
URANINITE-Continued

Nivenite

A variety of uraninite containing rare earths.

Dana VII, v. 1, p. 611, 613-615, 617.

Pitchblende

A colloform, fine-grained variety of uraninite.

Dana VII, v. 1, p. 611-619.

Ulrichite

Synonym of uraninite.

Dana VII, v. 1, p. 611, 613, 617.

Uranatemnite

Synonym of uraninite.

U.S. Geol. Survey Bull. 1064, p. 12 (1958).

Uranoniobite

Synonym of uraninite.

Dana VII, v. 1, p. 611, 613.

Uranopissite

Synonym of uraninite.

Mineralog. Mag., v. 16, p. 374 (1913).

\section{URANOCHALCITE}

An ill-defined uranium sulfate of doubtful validity.

Dana VII, v. 2, p. 600.

URANOCHER

A generic term used chiefly for uranium sulfates; also used for some uranium oxides.

Dana VII, v. 2, p. 581, 598.

\section{URANOPHANE}

$\mathrm{Ca}\left(\mathrm{UO}_{2}\right)_{2}\left(\mathrm{SiO}_{3}\right)_{2}(\mathrm{OH})_{2} \cdot 5 \mathrm{H}_{2} \mathrm{O}$

Dimorphous with beta-uranophane; isostructural with sklodowskite and cuprosklodowskite.

$\mathrm{U}=55.6$ percent.

Color: Yellow. A common secondary mineral.

Am. Mineralogist, v. 40, p. 634-645 (1955); v. 42, p. 594-618 (1957).

Lambertite

Synonym of uranophane.

Am. Mineralogist, v. 11, p. 155-157 (1926).

Uranotil (or uranotile)

Synonym of uranophane.

Dana VI, p. 699.

\section{URANOPILITE}

$\left(\mathrm{UO}_{2}\right)_{6}\left(\mathrm{SO}_{4}\right)(\mathrm{OH})_{10} \cdot 12 \mathrm{H}_{2} \mathrm{O}$

$\mathrm{U}=67.9$ percent.

Color: Yellow. A secondary mineral.

Dana VII, v. 2, p. 581-582.

Am. Mineralogist, v. 37, p. 950 (1952).

See also Meta-uranopilite, p. 26. 


\section{URANOSPATHITE}

$\mathrm{Cu}\left(\mathrm{UO}_{2}\right)_{2}\left(\mathrm{AsO}_{4}, \mathrm{PO}_{4}\right)_{2} \cdot 11 \mathrm{H}_{2} \mathrm{O}(?)$

$\mathrm{U}=46.0$ percent(?).

Color: Yellow to pale green. A rare secondary mineral.

Dana VII, v. 2, p. 990.

Mineralog. Mag., v. 30, p. 343-353 (1954).

URANOSPHAERITE (or uranospherite)

$\mathrm{Bi} \mathrm{UO}_{4}(\mathrm{OH})$

$\mathrm{U}=45.1$ percent.

Color: Orange to red. A rare secondary mineral.

Am. Mineralogist, v. 42, p. 905-908 (1957).

Soc. Française Minéralogie et Cristallographie Bull., v. 82, p. 265-268 (1959).

\section{URANOSPINITE}

$\mathrm{Ca}\left(\mathrm{UO}_{2}\right)_{2}\left(\mathrm{AsO}_{4}\right)_{2} \cdot 10 \mathrm{H}_{2} \mathrm{O}$

$\mathrm{U}=45.9$ percent.

Color: Yellow to green. A rare secondary mineral.

Dana VII, v. 2, p. 990-991.

Am. Mineralogist, v. 38, p. 1159-1168 (1953).

Calciumarsenuranit

Synonym of uranospinite.

Mineralog. Mag., v. 28, p. 732 (1949).

URGITE (or urhite, or urhyte)

$\mathrm{UO}_{3} \cdot n \mathrm{H}_{2} \mathrm{O}$

Possibly a mixture equivalent to "gummite."

$\mathrm{U}=58.9$ to 59.2 percent.

Color: Reddish yellow to amber yellow. A secondary mineral.

Am. Mineralogist, v. 42, p. 442 (1957).

\section{URSILITE}

$2(\mathrm{Ca}, \mathrm{Mg}) 0.2 \mathrm{UO}_{3} \cdot 5 \mathrm{SiO}_{2} \cdot 9-10 \mathrm{H}_{2} \mathrm{O}$

Calcium ursilite, with $\mathrm{Ca}$ predominant; magnesium ursilite, with $\mathrm{Mg}$ predominant.

$\mathrm{U}=41.4$ to $\mathbf{4 2 . 1}$ percent. Inadequately described.

Color: Lemon yellow. Occurs along joints in quartz porphyries.

The geology of uranium, 1958, p. 61-65, translated from Russian by Consultants Bur., Inc.

Am. Mineralogist, v. 44, p. 464-465 (1959).

USIGITE (or usihite, or usihyte)

$\mathrm{R} f\left(\mathrm{UO}_{2}\right)_{2} \mathrm{Si}_{2} \mathrm{O}_{7} \cdot n \mathrm{H}_{2} \mathrm{O}$

Inadequately described mineral.

Color: Yellow.

Am. Mineralogist, v. 43, p. 1008 (1958).

UVANITE

$\mathrm{U}_{2} \mathrm{~V}_{8} \mathrm{O}_{21} \cdot 15 \mathrm{H}_{2} \mathrm{O}(?)$

$\mathrm{U}=34.3$ percent(?)

Color: Brownish yellow. A rare secondary mineral.

Dana VII, v. 2, p. 1056.

†Meaning of $\mathrm{R}$ not specified. 
VANDENBRANDEITE

$\mathrm{CuUO}_{4} \cdot 2 \mathrm{H}_{2} \mathrm{O}$

$\mathrm{U}=59.3$ percent.

Color: Blackish green. A rare secondary mineral.

Dana VII, v. 1, p. 632-633.

Am. Mineralogist, v. 36, p. 394-410 (1951).

Uranolepidite

Synonym of vandenbrandeite.

Am. Mineralogist, v. 19, p. 235-236 (1934).

VANDENDRIESSCHEITE (or vandendriesscheite I)

$\mathrm{PbO} .7 \mathrm{UO}_{3} \cdot 12 \mathrm{H}_{2} \mathrm{O}(?)$

$\mathrm{U}=68.3$ percent.

Color: Yellow. A rare secondary mineral.

Am. Mineralogist, v. 18, p. 20-24 (1933).

Soc. Belge Géologie, Paléontologie, et Hydrologie Bull., v. 70, p. 212-225 (1947).

U.S. Geol. Survey Bull. 1064, p. 81-87 (1958).

Soc. Française Minéralogie et Cristallographie Bull., v. 82, p. 261-264 (1959).

Am. Mineralogist, v. 50, p. 1026-1061 (1960).

Metavandendriesscheite (or vandendriesscheite II)

A phase related to vandendriesscheite and perhaps a lower hydrate.

Am. Mineralogist, v. 50, p. 1026-1061 (1960).

VANURALITE

$\left(\mathrm{UO}_{2}\right)_{2} \mathrm{Al}\left(\mathrm{VO}_{4}\right)_{2}(\mathrm{OH}) \cdot 8 \mathrm{H}_{2} \mathrm{O}$

$\mathrm{U}=50.0$ percent.

Color: Citron yellow. A secondary mineral.

Acad. Sci. [Paris] Comptes Rendus, v. 256, p. 5374-5376 (1963).

Am. Mineralogist, v. 48, p. 1415-1416 (1963).

VANURANYLITE

$\left[\left(\mathrm{H}_{3} \mathrm{O}\right)_{1 \cdot 2}(\mathrm{Ba}, \mathrm{Ca}, \mathrm{K}, \mathrm{Pb})_{0.4}\right]\left(\mathrm{UO}_{2}\right)_{2}\left(\mathrm{VO}_{4}\right)_{2} \cdot 4.2 \mathrm{H}_{2} \mathrm{O}$

An oxonium member of the carnotite group.

$\mathrm{U}=48.5$ percent.

Color: Bright yellow. A secondary mineral.

Zapiski Vses. Mineralog. Obsheh. 94 (4) p. 463-465 (1965).

Am. Minerologist, v. 51, p. 1548 (1966).

VOGLIANITE

A hydrous calcium and uranium sulfate of doubtful validity.

Dana VII, v. 2, p. 600.

VOGLITE

$\mathrm{Ca}_{2} \mathrm{Cu}\left(\mathrm{UO}_{2}\right)\left(\mathrm{CO}_{3}\right)_{4} \cdot 6 \mathrm{H}_{2} \mathrm{O}$

$\mathrm{U}=31.3$ percent.

Color: Green. A rare secondary mineral.

Dana VII, v. 2, p. 237.

U.S. Geol. Survey Bull. 1064, p. 126-128 (1958).

Uran-Kalk-Kupfer-Carbonat

Synonym of voglite.

Reichsanst. Wien Geol. Jahrb., v. 4, p. 220 (1853). 


\section{WALPURGITE}

Probably $\mathrm{Bi}_{4}\left(\mathrm{UO}_{2}\right)\left(\mathrm{AsO}_{4}\right)_{2} \mathrm{O}_{4} \cdot 3 \mathrm{H}_{2} \mathrm{O}$

$\mathrm{U}=15.9$ percent.

Color: Yellow. A rare secondary mineral.

Dana VII, v. 2, p. 796-797.

Waltherite (or walpurgite)

The name waltherite (1857) has priority over walpurgite (1877), but the description of waltherite is so inadequate that the name should be dropped.

Chemie der Erde, v. 17, p. 341-345 (1955).

Am. Mineralogist, v. 41, p. 960 (1956); v. 42, p. 121 (1957).

\section{WEEKSITE}

$\mathrm{K}\left(\mathrm{UO}_{2}\right)_{2}\left(\mathrm{Si}_{2} \mathrm{O}_{5}\right)_{3} \cdot 4 \mathrm{H}_{2} \mathrm{O}$

$\mathrm{U}=43.4$ percent.

Color: Yellow. A secondary mineral.

Am. Mineralogist, v. 45, p. 39-52 (1960).

The "gastunite" described in Am. Mineralogist, v. 44, p. 1047-1056 (1959) is weeksite.

\section{WIDENMANNITE}

Carbonate of $\mathrm{U}$ and $\mathrm{Pb}$.

No analysis. Needs further study.

Color: Yellow. A secondary mineral.

Am. Mineralogist, v. 47, p. 415 (1962).

\section{WIIKITE}

Ill-defined material high in $\mathrm{Nb}, \mathrm{Ta}, \mathrm{Ti}$, and $\mathrm{Y}$, now found to be variable mixtures of euxenite and obruchevite.

$\mathrm{U}=\mathrm{up}$ to 13.2 percent; $\mathrm{Th}=$ up to 3.7 percent.

Finlande Comm. Géol. Bull. 82, p. 1-68 (1928); Bull. 115 p. 213-229 (1936); Bull. 149, p. 1-76 (1950).

Am. Mineralogist, v. 47, p. 812 (1962).

\section{Nuolaite}

A mixture similar to wiikite.

$\mathrm{Th}=1.8$ to 3.5 percent.

Dana VII, v. 1, p. 801.

\section{WÖLSENDORFITE}

$(\mathrm{Pb}, \mathrm{Ca}) \mathrm{U}_{2} \mathrm{O}_{7} \cdot 2 \mathrm{H}_{2} \mathrm{O}$

This is "Mineral C" of Frondel (1956), now a validated species.

$\mathrm{U}=58.1$ percent.

Color: Red, orange red. A secondary mineral.

Am. Mineralogist, v. 41, p. 561 (1957) ; v. 42, p. 919 (1957).

Soc. Française Minéralogie et Cristallographie Bull., v. 82, p. 256-257 (1959).

\section{WYARTITE}

$\mathrm{UO}_{2} \cdot 6 \mathrm{UO}_{3} \cdot 2 \mathrm{CO}_{2} \cdot 3 \mathrm{CaO} \cdot 12-14 \mathrm{H}_{2} \mathrm{O}$

This was described as ianthinite (not "ianthinite français" of Bignand), but is now recognized as a distinet species.

$\mathrm{U}=66.8$ to 67.7 percent.

Color: Violet black. Rare secondary mineral.

Am. Mineralogist, v. 40, p. $943-944$ (1955); v. 44, p. 908 (1959); v. 45, p. 200-208 (1960). 
YTTRIALITE

$(\mathrm{Y}, \mathrm{Th})_{2} \mathrm{Si}_{2} \mathrm{O}_{7}$

Possibly thalenite, with muchTh.

$\mathrm{U}=0.7$ to 4.0 percent; $\mathrm{Th}=4.6$ to 10.5 percent.

Color: Olive green. From granitic pegmatites.

Dana VI, p. 512.

Mineralog. Abs., v. 12, p. 531 (1955).

YTTROCRASITE

(Y, Th, U, Ca) ${ }_{2} \mathrm{Ti}_{4} \mathrm{O}_{11}(?)$

$\mathrm{U}=3.3$ percent; $\mathrm{Th}=7.3$ percent.

Color: Black. From granite pegmatite.

Dana VII, v. 1, p. 793.

Mineralog. Abs., v. 13, p. 565-566 (1958).

ZELLERITE

$\mathrm{Ca}\left(\mathrm{UO}_{2}\right)\left(\mathrm{CO}_{3}\right)_{2} \cdot 5 \mathrm{H}_{2} \mathrm{O}$

$\mathrm{U}=44.8$ percent.

Color: Lemon yellow. A secondary mineral.

Am. Mineralogist, v. 51, p. 1567-1578 (1966).

ZEUNERITE

$\mathrm{Cu}\left(\mathrm{UO}_{2}\right)_{2}\left(\mathrm{AsO}_{4}\right)_{2} \cdot 10-12 \mathrm{H}_{2} \mathrm{O}$

$\mathrm{U}=47.7$ to 49.5 percent.

Color: Green. A secondary mineral.

Am. Mineralogist, v. 42, p. 905-908 (1957).

Mineralog. Petrog. Mitt., v. 9. p. 111-135 (1964).

Kupferarsenuranit

Synonym of zeunerite.

Mineralog. Mag., v. 28, p. 732 (1949).

\section{ZIPPEITE}

$\mathrm{K}_{4}\left(\mathrm{UO}_{2}\right)_{6}\left(\mathrm{SO}_{4}\right)_{3}(\mathrm{OH})_{10} \cdot \mathrm{H}_{2} \mathrm{O}$

Related species contain $\mathrm{Na}, \mathrm{Co}, \mathrm{Ni}, \mathrm{Mg}, \mathrm{Fe}$, or $\mathrm{Zn}$.

$\mathrm{U}=63.4$ percent.

Color: Orange yellow. A secondary mineral.

Dana VII, v. 2, p. 598.

Am. Mineralogist, v. 37, p. 394, 950 (1952).

United Nations Internat. Conf. on Peaceful Uses of Atomic Energy, 2d, Geneva, Proc., v. 2, p. 278-285 „(1958).

Dauberite

Possibly a synonym of zippeite.

U.S. Geol. Survey Bull. 1064, p. 141 (1958).

\section{ZIRKELITE}

$(\mathrm{Ca}, \mathrm{Fe}, \mathrm{Th}, \mathrm{U})_{2}(\mathrm{Ti}, \mathrm{Nb}, \mathrm{Zr})_{2} \mathrm{O}_{l}($ ?)

$\mathrm{U}=1.4$ percent; $\mathrm{Th}=6.4$ percent.

Color: Black. From alkalic pyroxenite.

Dana VII, v. 1, p. 740. 


\section{B. MINERALS WITH MINOR AMOUNTS OF URANIUM AND THORIUM}

\section{ABUK UMALITE}

$(\mathrm{Y}, \mathrm{Th}, \mathrm{Ca})_{5}\left(\mathrm{SiO}_{4}, \mathrm{PO}_{4}, \mathrm{AlO}_{4}\right)_{3}(\mathrm{O}, \mathrm{F})$

Related to apatite.

$\mathrm{Th}=$ up to 0.8 percent.

Am. Mineralogist, v. 24, p. 66 (1939).

Strunz, Hugo, Mineralogische Tabellen, 4th ed., p. 291, Leipzig Akad. Verlags. (1966).

\section{ALLANITE}

$(\mathrm{Ca}, \mathrm{Ce}, \mathrm{Th})_{2}(\mathrm{Al}, \mathrm{Fe}, \mathrm{Mg})_{3} \mathrm{Si}_{3} \mathrm{O}_{12}(\mathrm{OH})$

$\mathrm{U}=$ up to 2.95 percent, but is usually only a few hundredths of a percent.

$\mathrm{Th}=$ up to 4.35 percent.

Dana VI, p. 522-526.

Am. Mineralogist, v. 36, p. 223-248 (1951); v. 42, p. 367-378 (1957); v. 49 , p. $1159-1178$ (1964).

Mineralog. Abs., v. 11, p. 434-435 (1952).

Soc. Française Minéralogie et Cristallographie Bull., v. 78, p. 257-261 (1955); v. 81, p. 150-153 (1958).

Akad. Nauk SSSR Doklady, v. 129, p. 916-918 (1959) (in Russian).

Bagrationite

Variety of allanite.

Name also refers to cerian variety of epidote.

Dana VI, p. 518-519.

Bodenite

Related to muromontite in composition.

Dana VI, p. 526.

Bucklandite

Synonym of allanite.

Dana VI, p. 522-523, 525.

Muromontite

Apparently related to allanite but contains yttrium and beryllium.

Dana VI, p. 526.

Nagatelite

Phosphatian variety of allanite.

Am. Mineralogist, v. 16, p. 343-344 (1931).

Orthite

Synonym of allanite.

Dana VI, p. 522-526.

Treanorite

Synonym of allanite.

Am. Mineralogist, v. 29, p. 456 (1954).

Uralorthite

Synonym of allanite.

Dana VI, p. 523-525. 


\section{ALLANITE-Continued}

Wasite

Altered allanite.

Dana VI, p. 526.

Xanthorite

Altered allanite.

Dana VI, p. 522-523, 525.

Yttro-orthite (or yttrium orthite)

Variety of allanite with high $\mathrm{Y}$ content.

Mineralog. Mag., v. 23, p. 639 (1934).

Am. Mineralogist, v. 49, p. 1159-1178 (1964).

\section{ANCYLITE}

$(\mathrm{Ce}, \mathrm{La})(\mathrm{Sr}, \mathrm{Ca})\left(\mathrm{CO}_{3}\right)_{2}(\mathrm{OH}) \cdot \mathrm{H}_{2} \mathrm{O}$

$\mathrm{Th}=0.18$ percent.

Heinrich, E. W., Mineralogy and geology of radioactive raw materials, 1958, p. 127.

\section{ANGLESITE}

$\mathrm{PbSO}_{4}$

A highly radioactive anglesite, with activity presumably due to radium, has been reported.

Great Britain Geol. Survey, Summary of Progress for 1957, p. 59 (1958).

Compare Barite, p. 52.

\section{APATITE}

$\mathrm{Ca}_{5}\left(\mathrm{PO}_{4}\right)_{3}(\mathrm{~F}, \mathrm{OH}, \mathrm{Cl})$

$\mathrm{U}=\mathrm{up}$ to 0.08 percent in apatite from igneous rocks, but is usually less than 0.01 percent; $\mathrm{Th}=\mathrm{up}$ to 0.003 percent.

U.S. Geol. Survey Prof. Paper 314-D, p. 45-90 (1958).

Adams, J. A. S., Osmond, J. K. and Rogers, J. J. W., The geochemistry of thorium and uranium in Ahrens, L. H. and others, Physies and chemistry of the earth: v. 3, London, Pergamon Press, p. 314 (1959).

Carbonate-fluorapatite

A carbonatian variety of apatite, the principal constituent of marine phosphorites, concretions, and fossil bone.

$\mathrm{U}=$ up to 0.85 percent, more commonly $0.005-0.02$ percent.

U.S. Geol. Survey Prof. Paper 314-D, p. 45-90 (1958).

\section{Uran-apatite}

A uranoan apatite.

U reported to be 1.4-3.3 percent. Needs further study.

Soc. Française minéralogie et Cristallographie Bull., v'. 81, p. 337 (1958).

\section{BADDELEYITE}

$\mathrm{ZrO}_{2}$ with minor $\mathrm{Hf}, \mathrm{Ti}$, and $\mathrm{Fe}^{+3}$.

May contain appreciable Th and minor $\mathrm{U}$.

Heinrich, E. W., Mineralogy and geology of radioactive raw materials, 1958, p. 125.

\section{Brazilite}

Mixture of baddeleyite, zircon, and altered zircon.

$\mathrm{U}=\mathrm{up}$ to 0.8 percent.

Heinrich, E. W., Mineralogy and geology of radioactive raw materials, 1958 , p. 125. 


\section{BADDELEYITE-Continued}

Caldasite

Mixture similar to brazilite.

Heinrich, E. W., Mineralogy and geology of radioactive raw materials, 1958, p. 125.

\section{Zirkite}

Mixture similar to brazilite.

Heinrich, E. W., Mineralogy and geology of radioactive raw materials, 1958 , p. 125.

\section{BARITE}

$(\mathrm{Ba}, \mathrm{Pb}, \mathrm{Ra}) \mathrm{SO}_{4}$

Anglesobarite

Synonym of hokutolite.

Dana VII, v. 2, p. 408, 411.

Hokutolite

Plumboan variety of barite. Radioactive.

Probably contains Ra, Th, U.

Dana VII, v. 2, p. 408, 411.

Radiobarite

Synonym of hokutolite.

Heinrich, E. W., Mineralogy and geology of radioactive raw materials, 1958, p. 127.

\section{BASTNAESITE}

$(\mathrm{Ce}, \mathrm{La}) \mathrm{FCO}_{3}$

$\mathrm{U}$ and Th present, but usually less than 1 percent.

Dana VII, v. 2, p. 289-291.

Am. Mineralogist, v. 38, p. 932-963 (1953).

See also Thorbastnaesite, p. 37.

Buszite

Synonym of bastnaesite.

Am. Mineralogist, v. 39, p. 406 (1954).

Hydroxyl-bastnaesite

$(\mathrm{Ce}, \mathrm{La})\left(\mathrm{CO}_{3}\right)(\mathrm{OH}, \mathrm{F})$

$\mathrm{Th}=\mathbf{0 . 4 4}$ percent.

Color: Waxy yellow to dark brown.

Am. Mineralogist, v. 50, p. 805 (1965).

\section{BRITHOLITE}

$(\mathrm{Ca}, \mathrm{Ce})_{5}\left[(\mathrm{Si}, \mathrm{P}) \mathrm{O}_{4}\right]_{3}(\mathrm{OH}, \mathrm{F})$

Apatite group.

$\mathrm{U}=$ up to 1.9 percent; $\mathrm{Th}=$ up to 17 percent but is usually less than 2 percent.

Heinrich, E. W., Mineralogy and geology of radioactive raw materials 1958, p. 148.

Compare Abukumalite, p. 50.

See also Fenghuanglite, p. 18. 


\section{BRITHOLITE-Continued}

Alumobritholite

An aluminum-rich britholite.

$\mathrm{Th}=$ up to 8.0 percent.

Color: Yellowish brown, slightly green. Occurs in pegmatites.

Am. Mineralogist, v. 46, p. 1514-1515 (1961).

Pravdite

An altered britholite.

Am. Mineralogist, v. 48, p. 709 (1963); v. 49, p. 1501 (1964).

\section{CAPPELENITE}

$(\mathrm{Ba}, \mathrm{Ca}, \mathrm{Ce}, \mathrm{La}, \mathrm{Na})_{3}\left(\mathrm{BO}_{3}\right)_{2}\left(\mathrm{SiO}_{3}\right)$

$\mathrm{Th}=$ up to 0.71 percent.

Strunz, Hugo, Mineralogische Tabellen, 4th ed., p. 359, Leipzig Akad. Verlags. (1966).

Heinrich, E. W., Mineralogy and geology of radioactive raw materials, 1958, p. 148.

CERITE

A cerium silicate with minor $\mathrm{Ca}$ and Fe. Formula uncertain.

$\mathrm{U}=0.4$ percent; $\mathrm{Th}=0.3$ percent.

Am. Mineralogist, v. 24, p. 381-404 (1940).

See also Hydrocerite, p. 21.

\section{CHINGLUSUITE}

A complex silicate of $\mathrm{Na}, \mathrm{Mn}, \mathrm{Ca}$, and $\mathrm{Ti}$, and contains small amounts of $\mathrm{Th}, \mathrm{U}$, and rare earths.

$\mathrm{Th}=0.05$ percent; $\mathrm{U}=$ up to 0.39 percent.

Mineralog. Abs., v. 7, p. 222 (1938).

Heinrich, E. W., Mineralogy and geology of radioactive raw materials, 1958, p. 148.

\section{CORDYLITE}

$(\mathrm{Ce}, \mathrm{La})_{2} \mathrm{Ba}\left(\mathrm{CO}_{3}\right) \mathrm{F}_{2}$

$\mathrm{Th}=0.26$ percent.

Dana VII, v. 2, p. 285-287.

\section{CRANDALLITE}

$\mathrm{CaAl}_{3}\left(\mathrm{PO}_{4}\right)_{2}(\mathrm{OH})_{5} \cdot \mathrm{H}_{2} \mathrm{O}$

$\mathrm{U}=$ up to 0.06 percent.

U.S. Geol. Survey Prof. Paper 300, p. 495-504 (1956).

French West Africa, Serv. Geol. et Prospect. Minière Bull. 25, p. 1-84 (1959).

Pseudowavellite

Synonym of crandallite.

\section{DOVERITE}

$\mathrm{YFCO}_{3} \cdot \mathrm{CaCO}_{3}(?)$

Yttrium analog of synchysite(?).

$\mathrm{U}=0.18$ percent; $\mathrm{Th}=1.4$ percent, but monazite was present.

Am. Mineralogist, v. 45, p. 92-98 (1960); v. 47, p. 337-343 (1962).

\section{EPIDOTE}

$\mathrm{Ca}_{2}(\mathrm{Al}, \mathrm{Fe})_{3}\left(\mathrm{SiO}_{4}\right)_{3}(\mathrm{OH})$

$\mathrm{U}=$ up to 0.14 percent; $\mathrm{Th}=\mathrm{up}$ to 0.05 percent.

Am. Geophys. Union Trans., v. 38, p. 939-944 (1957).

Akad. Nauk SSSR Doklady, v. 127, p. 865-868 (1959) (in Russian). 


\section{ERIKITE}

$(\mathrm{La}, \mathrm{Ce})_{x}(\mathrm{P}, \mathrm{Si}) \mathrm{O}_{4} \cdot \mathrm{H}_{2} \mathrm{O}$

$\mathrm{Th}=0.28$ to 1.01 percent.

Color: Yellow green, greenish yellow. Occurs in sodalite syenite pegmatites.

The type erikite from Greenland has been shown to be monazite (Am. Mineralogist, v. 44, p. 1329 (1959)), but the Kola Peninsula mineral appears to be different.

Perhaps a silicatian rhabdophane.

Vlasov, K. A., Kuz'menko, M. V. and Es'kova, E. M., The Lovozero alkaline massif, 1959, p. 425-427, Izdatel. Akad. Nauk SSSR, Moscow (in Russian).

Am. Mineralogist, v. 47, p. 419-420 (1962).

FERSMITE

$(\mathrm{Ca}, \mathrm{Ce}, \mathrm{Na})(\mathrm{Nb}, \mathrm{Ti}, \mathrm{Fe}, \mathrm{Al})_{2}(\mathrm{O}, \mathrm{OH}, \mathrm{F})_{6}$

$\mathrm{U}=0.02$ percent; $\mathrm{Th}=\mathbf{0 . 4 2}$ percent.

Am. Mineralogist, v. 32, p. 373 (1947); v. 44, p. 1-8 (1959).

FLORENCITE

$\mathrm{CeAl}_{3}\left(\mathrm{PO}_{4}\right)_{2}(\mathrm{OH})_{6}$

Radioactivity derived from thorium present.

South Australia Dept. Mines Geol. Survey Bull. 30, p. 132 (1954).

GADOLINITE

$\mathrm{Be}_{2} \mathrm{FeY}_{2} \mathrm{Si}_{2} \mathrm{O}_{10}$

$\mathrm{U}=$ up to 0.25 percent; $\mathrm{Th}=$ up to 1.5 percent.

Heinrich, E. W., Mineralogy and geology of radioactive raw materials 1958, p. 142.

Calciogadolinite

Variety of gadolinite.

Heinrich, E. W., Mineralogy and geology of radioactive raw materials, 1958 , p. 142.

\section{GAGARINITE}

$\mathrm{Na}_{2} \mathrm{Ca}_{2} \mathrm{Y}_{3}(\mathrm{~F}, \mathrm{Cl})_{1 \mathrm{~s}}$

$\mathrm{Th}=0.44$ percent.

Am. Mineralogist, v. 47, p. 805 (1962).

HELLANDITE

Near $\mathrm{Ca}_{3} \mathrm{Y}_{4} \mathrm{~B}_{4} \mathrm{Si}_{6} \mathrm{O}_{27} \cdot 3 \mathrm{H}_{2} \mathrm{O}$

$\mathrm{Th}=\mathbf{0 . 5 4}$ to 1.28 percent.

Heinrich, E. W., Mineralogy and geology of radioactive raw materials, 1958 , p. 148.

\section{HIBONITE}

(Ca, R.E.*)(Al, $\left.\mathrm{Fe}^{+3}, \mathrm{Ti}, \mathrm{Mg}\right)_{12} \mathrm{O}_{10}$

$\mathrm{U}=0.01$ percent or $\mathrm{Th}=0.1$ percent, from radioactivity.

Am. Mineralogist, v. 42, p. 119 (1957).

KARNASURTITE

$(\mathrm{Ce}, \mathrm{La}, \mathrm{Th})(\mathrm{Ti}, \mathrm{Nb})(\mathrm{Al}, \mathrm{Fe})(\mathrm{Si}, \mathrm{P})_{2} \mathrm{O}_{7}(\mathrm{OH})_{4} \cdot 3 \mathrm{H}_{2} \mathrm{O}$

Amorphous, gives a monazite-like $\mathrm{X}$-ray pattern when heated.

$\mathrm{Th}=2.48$ to 4.47 percent.

Color: Honey to pale yellow. Occurs in sodalite syenite pegamtites.

Am. Mineralogist, v. 45, p. 1133-1134 (1960); v. 47, p. 419-420 (1962).

\footnotetext{
*Rare earths.
} 


\section{KARNASURTITE-Continued}

Kozhanovite

Synonym of karnasurtite.

KOLM

Material resembling oil shale.

Form in which uranium is present is unknown.

$\mathrm{U}=\mathbf{0 . 4 3}$ percent.

Washington Acad. Sci. Jour., v. 21, p. 409-414 (1931).

\section{LOPARITE}

$(\mathrm{Na}, \mathrm{Ce}, \mathrm{Th})_{1-x}(\mathrm{Ti}, \mathrm{Nb})\left[\mathrm{O}_{3-x}(\mathrm{OH})_{x}\right]$

A member of the perovskite group.

$\mathrm{U}=$ up to 0.17 percent; $\mathrm{Th}=$ usually less than 1 percent. (For exception see irinite below.)

Color: Brown to black. Occurs in nepheline syenite pegmatite.

Dana VII, v. 1, p. 732-733.

Heinrich, E. W., Mineralogy and geology of radioactive raw materials, 1958, p. 125-126.

\section{Irinite}

A thorian loparite.

$\mathrm{Th}=11.4$ percent.

Akad. Nauk SSSR Doklady, v. 97, p. 725-728 (1954).

Am. Mineralogist, v. 40, p. 369 (1955).

Nioboloparite

A niobian loparite.

$\mathrm{Th}=1.0$ percent.

Am. Mineralogist, v. 43, p. 792 (1958).

\section{LOVOZERITE}

A complex silicate of $\mathrm{Ti}$ and $\mathrm{Zr}$.

$\mathbf{T h}=\mathbf{0 . 5 0}$ percent.

Mineralog. Abs., v. 7, p. 468-469 (1940).

\section{LUESHITE}

$(\mathrm{Na}, \mathrm{Ca}, \mathrm{Ce})(\mathrm{Nb}, \mathrm{Ti}) \mathrm{O}_{3}$

A member of the perovskite group.

$\mathrm{Th}=$ up to 2.9 percent.

Akad. Nauk SSSR Doklady, v. 171, p. 956-959 (1966).

MELANOCERITE

Chiefly a borosilicate of the Ce and $\mathrm{Y}$ metals.

$\mathrm{Th}=1.5$ percent.

Dana VI, p. 414-415.

See also Caryocerite, p. 14.

\section{MILLISITE}

$(\mathrm{Na}, \mathrm{K}) \mathrm{CaAl}_{6}\left(\mathrm{PO}_{4}\right)_{4}(\mathrm{OH})_{9} \cdot 3 \mathrm{H}_{2} \mathrm{O}$

$\mathrm{U}=0.03$ to 0.04 percent.

U.S. Geol. Survey Prof. Paper 300, p. 495-504 (1956).

\section{Pallite}

A ferrian variety of millisite.

$\mathrm{U}=0.01$ to 0.015 percent.

Acad. Sci. [Paris] Comptes Rendus, v. 239, p. 288-290 (1954).

Am. Mineralogist, v. 45, p. 256-257 (1960). 


\section{MOSANDRITE}

General formula $\mathrm{Na}_{2} \mathrm{Ca}_{4} \mathrm{CeTiSi}_{4} \mathrm{O}_{10}(\mathrm{~F}, \mathrm{OH})_{3}$

$\mathrm{U}=0.03$ percent; $\mathrm{Th}=$ up to 0.7 percent.

Am. Mineralogist, v. 43, p. 795-796 (1958).

Johnstrupite

Synonym of mosandrite.

Am. Mineralogist, v. 43, p. 795-796 (1958).

Lovchorrite

Synonym of mosandrite.

Am. Mineralogist, v. 43, p. 795-796 (1958).

Rinkite

Synonym of mosandrite.

Am. Mineralogist, v. 43, p. 795-796 (1958).

Rinkolite

Synonym of mosandrite.

Am. Mineralogist, v. 43, p. 795-796 (1958).

Vudyavrite

Final alteration product of mosandrite.

Mineralog. Abs. v. 13, p. 654 (1958).

\section{NATRONIOBITE}

$\mathrm{NaNbO}_{3}$

Apparently a dimorph of lueshite.

$\mathrm{Th}=0.49$ percent. Occurs in dolomitic carbonatites.

Am. Mineralogist, v. 47, p. 1483 (1962).

NIOBO-AESCHYNITE

$(\mathrm{Ce}, \mathrm{Y}, \mathrm{Ca}, \mathrm{Fe}, \mathrm{TH})(\mathrm{Nb}, \mathrm{Ti})_{2} \mathrm{O}_{6}$

Like aeschynite but with $\mathrm{Nb}$ greater than $\mathrm{Ti}$.

$\mathrm{Th}=2.2$ percent.

Color: Black. Occurs in quartz-arfvedsonite veinlets.

Am. Mineralogist, v. 47, p. 417 (1962).

\section{PANDAITE}

$(\mathrm{Ba}, \mathrm{Sr})(\mathrm{Nb}, \mathrm{Ti})(\mathrm{O}, \mathrm{OH})_{7}$, a member of the pyrochlore group.

$\mathrm{U}=0.3$ percent; $\mathrm{Th}=0.5$ to 0.7 percent.

Mineralog. Mag., v. 32, p. 10-25 (1959).

\section{PARISITE}

$(\mathrm{Ce}, \mathrm{La})_{2} \mathrm{Ca}\left(\mathrm{CO}_{3}\right)_{3} \mathrm{~F}_{2}$

$\mathrm{U}=0.002$ percent; $\mathrm{Th}=$ trace.

Heinrich, E. W., Mineralogy and geology of radioactive raw materials, 1958, p. 127.

\section{POLYMIGNYTE}

$A B \mathrm{O}_{4}$, where $A=\mathrm{Ca}, \mathrm{Fe}^{+2}, \mathrm{Y}, \mathrm{Zr}, \mathrm{Th} ; B=\mathrm{Nb}, \mathrm{Ti}, \mathrm{Ta}, \mathrm{Fe}^{+3}$.

$\mathrm{Th}=3.4$ percent.

Dana VII, v. 1, p. 764-766.

Heinrich, E. W., Mineralogy and geology of radioactive raw materials, 1958, p. 44. 


\section{POWELLITE}

$\mathrm{Ca}(\mathrm{Mo}, \mathrm{W}) \mathrm{O}_{4}$

Most analyses show no $U$, but up to 0.57 percent $U$ has been reported.

Dana VII, v. 2, p. 1079-1081.

United Nations Internat. Conf. on Peaceful Uses of Atomic Energy, 2d, Geneva, Proc. v. 2, p. 306-307 (1958).

Compare Wulfenite, p. 58.

\section{RHABDOPHANE}

$$
\text { (Ce,La) } \mathrm{PO}_{4} \cdot \mathrm{H}_{2} \mathrm{O}
$$

See also Erikite, p. 54; grayite, p. 20; ningyoite, p. 29; saryarkite, p. 35.

Silicorhabdophane

A variety of rhabdophane with high $\mathrm{SiO}_{2}$.

$\mathrm{T} h=0.28$ percent.

Am. Mineralogist, v. 47, p. 419-420 (1962).

\section{RIJKEBOERITE}

A barium analog of microlite.

$\mathrm{Ba}_{1-x}(\mathrm{Ta}, \mathrm{Nb})_{2}\left(\mathrm{O}, \mathrm{OH}, \mathrm{H}_{2} \mathrm{O}\right)_{7}$

$\mathrm{U}=1.45$ percent; $\mathrm{Th}=0.17$ percent.

Am. Mineralogist, v. 48, p. 1415 (1963).

ROWLANDITE

$(\mathrm{Y}, \mathrm{Ce}, \mathrm{Fe})_{8}\left(\mathrm{SiO}_{4}\right)_{2}(\mathrm{~F}, \mathrm{OH})(?)$

Possibly a metamict thalenite.

$\mathrm{U}=0.4$ percent.

Dana VI, p. 1047.

Strunz, Hugo, Mineralogische Tabellen., 4th ed., 1966, p. 339, Leipzig Akad. Verlag., $560 \mathrm{p}$.

\section{SPENCITE}

Perhaps (Y, Ce, $\mathrm{Ca}, \mathrm{Th})_{4} \mathrm{~B}_{3} \mathrm{Si}_{6} \mathrm{O}_{13}$

$\mathrm{Th}=2.1$ to 2.2 percent; $\mathrm{U}=0.75$ percent.

Canadian Mineralogist, v. 6, p. 576-581 (1961).

Am. Mineralogist, v. 47, p. 9-25 (1962).

\section{SPHENE}

$\mathrm{CaTiSi}(\mathrm{O}, \mathrm{OH}, \mathrm{F})_{5}$

$\mathrm{U}=\mathrm{up}$ to 0.14 percent; $\mathrm{Th}=\mathrm{up}$ to 0.65 percent.

Am. Geophys. Union Trans., v. 38, p. 939-944 (1957).

Titanite

Synonym of sphene.

STILLWELLITE

$(\mathrm{Ca}, \mathrm{Ce})(\mathrm{Si}, \mathrm{Al}, \mathrm{P}) \mathrm{B}(\mathrm{O}, \mathrm{OH}, \mathrm{F})_{5}$

$\mathrm{U}=0.19$ percent; $\mathrm{Th}=0.05$ percent.

Heinrich, E. W., Mineralogy and geology of radioactive raw materials, 1958, p. 148.

\section{SYNCHYSITE}

(Ce, $\mathrm{La}) \mathrm{Ca}\left(\mathrm{CO}_{3}\right)_{2} \mathrm{~F}$

$\mathrm{Th}=0.26$ percent.

Heinrich, E. W., Mineralogy and geology of radioactive raw materials, 1958 , p. 127. 


\section{TENGERITE}

$\mathrm{CaY}_{3}\left(\mathrm{CO}_{3}\right)_{4}(\mathrm{OH})_{3} \cdot 3 \mathrm{H}_{2} \mathrm{O}(?)$

The originally described tengerite (Dana VI, p. 306-307) is a different mineral, supposedly beryllium yttrium carbonate; no published analysis.

$\mathrm{Th}=0.3$ percent.

Dana VII, v. 2, p. 275-276.

Heinrich, E. W., Mineralogy and geology of radioactive raw materials, 1958, p. 127.

\section{THALENITE}

$\mathrm{Y}_{2} \mathrm{Si}_{2} \mathrm{O}_{7}$

Related to yttrialite.

$\mathrm{Th}=0.16$ percent.

Dana VI, app. I, p. 68.

TUNDRITE

$\mathrm{Ce}_{2} \mathrm{Ti}(\mathrm{Si}, \mathrm{P})(\mathrm{O}, \mathrm{OH})_{7} \cdot 4 \mathrm{H}_{2} \mathrm{O}$

Further work is needed on this mineral.

$\mathrm{Th}=0.69$ percent.

Am. Mineralogist, v. 47, p. 419-420 (1962); v. 50, p. 2097-2098 (1965).

Titanorhabdophane

Synonym of tundrite.

\section{TURANITE}

Supposedly $\mathrm{Cu}_{5}\left(\mathrm{VO}_{4}\right)_{2}(\mathrm{OH})_{4}$

May be related to mottramite.

Reported to contain 3.2 percent $\mathrm{U}$.

Dana VII, v. 2, p. 818.

\section{VANOXITE}

Perhaps $2 \mathrm{~V}_{2} \mathrm{O}_{4} \cdot \mathrm{V}_{2} \mathrm{O}_{5} \cdot 8 \mathrm{H}_{2} \mathrm{O}$

Reported to contain up to 0.42 percent $U$; species may be invalid; analysis was made on a mixture (Weeks, A. D., written commun., 1960).

Dana VII, v. 1, p. 601-602.

\section{VESUVIANITE (or idocrase)}

$\mathrm{Ca}_{10}(\mathrm{Mg}, \mathrm{Fe})_{2} \mathrm{Al}_{4}\left(\mathrm{SiO}_{4}\right)_{5}\left(\mathrm{Si}_{2} \mathrm{O}_{7}\right)_{2}(\mathrm{OH})_{4}$

Not usually reported to contain $\mathrm{U}$ and $\mathrm{Th}$, but a metamict variety is reported from nepheline syenite pegmatite.

$\mathrm{U}=0.85$ percent; $\mathrm{Th}=0.47$ percent.

Akad. Nauk SSSR Doklady, v. 130, p. 400-403 (1960).

\section{W ULFENITE}

$\mathrm{Pb}(\mathrm{Mo}, \mathrm{U}) \mathrm{O}_{4}$

Most analyses show no $U$, but up to 9.65 percent $U$ has been reported. Color: Orange, yellow, brown. A secondary mineral.

Zapiski Vses. Mineralog. Obshch., v. 89, p. 180-186 (1960) (in Russian).

See also Powellite, p. 57.

\section{XENOTIME}

$\mathrm{YPO}_{4}$

$\mathrm{U}=$ up to 3.6 percent: $\mathrm{Th}=$ up to 2.2 percent.

Dana VII, v. 2, p. 688-691. 


\section{YTTROTANTALITE}

$(\mathrm{Fe}, \mathrm{Y}, \mathrm{U})(\mathrm{Nb}, \mathrm{Ta}) \mathrm{O}_{4}$

Grades to samarskite.

$\mathrm{U}=$ as much as 4.8 percent; $\mathrm{Th}=$ as much as 2.2 percent.

Dana VII, v. 1, p. 763-764.

Mineralog. Abs., v. 11, p. 231-232 (1950).

Mineralog. Mag., v. 31 p. 763-780 (1958).

Yttrocolumbite

Similar to yttrotantalite.

Am. Mineralogist, v. 25, p. 155 (1940).

YTTROTUNGSTITE (or thorotungstite)

$\mathrm{Y}_{2} \mathrm{~W}_{5} \mathrm{O}_{18} \cdot 4 \mathrm{H}_{2} \mathrm{O}$

$\mathrm{Th}=$ up to 0.07 percent.

Akad. Nauk SSSR Doklady, v. 163, p. 447-449 (1965) (in Russian).

\section{ZIRCON}

$\mathrm{ZrSiO}_{4}$

$\mathrm{U}$ and Th low in most specimens, but as much as 2.7 percent $\mathrm{U}$ and $\mathbf{1 3 . 1}$ percent $\mathrm{Th}$ reported.

Dana VI, p. 482-486.

Alvite

Variety of zircon near cyrtolite.

Dana VI, p. 487-488.

Arshinovite

Variety of zircon.

$\mathrm{U}=$ several tenths of one percent.

Razumnaya, E. G., Smolianskaya, G. A., Korolev, K. G., and Pakulpis, G. V., United Nations Internat. Conf. on Peaceful Uses of Atomic Energy, 2d., Geneva, Proc. v. 2. p. 307-308 (1958).

Am. Mineralogist, v. 44, p. 210 (1959).

Azorite

Variety of zircon.

Dana VI, p. 482-484.

Calyptolite

Probably altered zircon.

Dana VI, p. 482, 486.

\section{Cyrtolite}

Altered zircon, containing $\mathrm{U}, \mathrm{Th}, \mathrm{Y}$ and other rare earths.

Dana VI, p. 487.

Am. Mineralogist, v. 38, p. 1007-1018 (1953).

Hagatalite

Synonym of zircon.

Am. Mineralogist, v. 11, p. 137 (1926).

Hoegtveitite

May be alvite.

Am. Mineralogist, v. 12, p. 97 (1927).

Malacon

Hydrated or altered zircon.

Dana VI, p. 486. 


\section{ZIRCON-Continued}

Naegite

Variety of zircon.

Mineralog. Abs., v. 2, p. 36 (1923).

Oerstedite

Altered zircon.

Dana VI, p. 486.

Oyamalite

Variety of zircon.

Am. Mineralogist, v. 11, p. 137-138 (1926).

Ribeirite

An yttrian zircon.

Mineralog. Abs., v. 12, p. 305 (1954).

Am. Mineralogist, v. 39, p. 159 (1954); v. 41, p. 168 (1956).

Tachyaphaltite

Probably altered zircon.

Dana VI, p. 486.

Yamagutilite (or yamaguchilite)

Variety of zircon.

Contains $\mathrm{P}_{2} \mathrm{O}_{5}$ and rare earths.

Mineralog. Mag., v. 24, p. 626 (1937).

\section{ZIRCONOLITE}

$(\mathrm{Ca}, \mathrm{Ce}) \mathrm{Zr}(\mathrm{Ti}, \mathrm{Nb})_{2} \mathrm{O}_{7}$

$\mathrm{U}=\mathrm{up}$ to 1.49 percent; $\mathrm{Th}=\mathrm{up}$ to 0.51 percent.

Am. Mineralogist, v. 42, p. 581-582 (1957).

Niobozirconolite

A niobium-rich zirconolite.

$\mathrm{U}=0.34$ percent, $\mathrm{Th}=2.5$ percent.

Am. Mineralogist, v. 46, p. 465 (1961). 


\section{MINERALS REPORTED TO CONTAIN URANIUM AND THORIUM MINERALS AS IMPURITIES OR INTERGROWTHS}

ADAMITE

$\mathrm{Zn}_{2}(\mathrm{OH})\left(\mathrm{AsO}_{4}\right)$

Reported to contain small amounts of $\mathrm{U}$.

Mineralog. Abs., v. 10, p. 375 (1948).

\section{ALLOPHANE}

$\mathrm{Al}_{2} \mathrm{O}_{3} \cdot \mathrm{SiO}_{2} \cdot n \mathrm{H}_{2} \mathrm{O}$

Reported by R. G. Coleman (written commun., 1960) to contain up to 0.91 percent $U$.

Compare Evansite, p. 62; opal, p. 63.

\section{BOBENBENDERITE}

Mixture of fluorite and spessartite.

$\mathrm{U}=0.11$ percent.

Am. Mineralogist, v. 34, p. 608-611 (1948).

\section{CALCIOVOLBORTHITE}

$\mathrm{CaCu}\left(\mathrm{VO}_{4}\right)(\mathrm{OH})$

Reported to contain 3.1 percent $U$.

Dana VII, v. 2, p. 816-818.

Soc. Française Minéralogie et Cristallographie Bull., v. 79, p. 246-250.

Tangeite (or tangueite)

Synonym of calciovolborthite.

\section{CHR YSOCOLLA}

$\mathrm{CuSiO}_{3} \cdot 2 \mathrm{H}_{2} \mathrm{O}$

$\mathrm{U}=\mathbf{0 . 0 3}$ percent.

Heinrich, E. W., Mineralogy and geology of radioactive raw materials, 1958, p. 530.

Katangite

Synonym of chrysocolla.

May contain $U$ in admixture.

Mineralog. Abs., v. 9, p. 23 (1946).

\section{COLUMBITE}

$(\mathrm{Fe}, \mathrm{Mn})(\mathrm{Nb}, \mathrm{Ta})_{2} \mathrm{O}_{6}$

$\mathrm{U}=$ up to 9.8 percent.

Dana VII, v. 1, p. 780-785.

Baierite (or baierine)

Synonym of columbite.

Dana VII, v. 1, p. 780.

Dianite

Synonym of columbite.

Dana VII, v. 1, p. 780. 


\section{COLUMBITE-Continued}

Ferrocolumbite

Synonym of columbite.

Dana VII, v. 1, p. 780, 783.

Ferro-ilmenite

Synonym of columbite.

Dana VII, v. 1, p. 780, 785.

Greenlandite

Synonym of columbite.

Dana VII, v. 1, p. 780, 784 .

Hermannolite

Synonym of columbite.

Dana VII, v. 1, p. 780, 785.

Manganocolumbite

Variety of columbite.

Dana VII, v. 1, p. 700, 783-784.

\section{CORVUSITE}

$\mathrm{V}_{2} \mathrm{O}_{4} \cdot 6 \mathrm{~V}_{2} \mathrm{O}_{5} \cdot x \mathrm{H}_{2} \mathrm{O}$

$\mathrm{U}=$ up to 2.5 percent.

Work in progress indicates species may be valid.

Reexamination of type material shows presence of rauvite, which might account for reported uranium content (Weeks, A. D., written commun., March 1952).

Am. Mineralogist, v. 18, p. 195-205 (1933); v. 44, p. 330-333 (1959).

\section{CRYPTOMELANE}

$(\mathrm{K}, \mathrm{Ba})(\mathrm{Mn}, \mathrm{Fe})_{8} \mathrm{O}_{16}$

Reported to contain up to 1 percent $U$.

Neues Jahrb. Mineralogie Monatsh., 1958, p. 73-84.

New Mexico Bur. Mines and Mineral Resources Mem. 15, p. 21-37 (1963).

\section{EVANSITE}

$\mathrm{Al}_{3}(\mathrm{OH})_{6} \mathrm{PO}_{4} \cdot 6 \mathrm{H}_{2} \mathrm{O}$

Reported to contain small amounts of $\mathrm{U}$ and $\mathrm{Th}$.

Mineralog. Abs., v. 10, p. 375 (1948).

Soc. Geol. Belgique Annales, v. 80, p. 497-501 (1957).

\section{FLUORITE}

$\mathrm{CaF}_{2}$

Some specimens, especially deep purple ones, are radioactive. This generally can be shown to be due to inclusions of uranium minerals, but the fluorite itself possibly may have $U$ in substitution for $\mathrm{Ca}$.

Dana VII, v. 2, p. 28-37.

Strong alpha bombardment produces the semiopaque black variety antozonite, as in the inner bands of halos surrounding uranininte and thorite inclusions. Centered thorium halos in fluorite have a maximum radius of about 41 microns. Uranium halos are smaller with a maximum radius of about 31 microns.

Am. Mineralogist, v. 37, p. 659-66, 910-930 (1952). 


\section{HYDROZINCITE}

$2 \mathrm{ZnCO} \cdot 3 \mathrm{Zn}(\mathrm{OH})_{2}$

$\mathrm{U}=0.07$ percent.

Heinrich, E. W., Mineralogy and geology of radioactive raw materials, 1958 , p. 530.

\section{LIMONITE}

Cryptocrystalline goethite, $\mathrm{FeO}(\mathrm{OH})$, with adsorbed or capillary water. $\mathrm{U}=\mathbf{0 . 0 3}$ percent.

Heinrich, E. W., Mineralogy and geology of radioactive raw materials, 1958, p. 530.

OPAL

$\mathrm{SiO}_{2} \cdot n \mathrm{H}_{2} \mathrm{O}$

Some varieties, particularly hyalite, show a green fluorescence due to uranium content.

$\mathrm{U}=$ usually 0.05 percent; however, up to 0.9 percent $\mathrm{U}$ has been reported in silica sinters from hot springs.

Mineralog. Abs., v. 10, p. 374-375 (1948).

Geol. Landesamt. Baden-Württemberg Abhandl., v. 3, p. 46, (1959).

PYROMORPHITE

$\mathrm{Pb}_{5}\left(\mathrm{PO}_{4}\right)_{3} \mathrm{Cl}$

Some specimens are uraniferous.

Zeitschr. Kristallographie, v. 62, p. 177-178 (1925).

SCAPOLITE

$(\mathrm{Na}, \mathrm{Ca})_{4} \mathrm{Al}_{3}\left(\mathrm{Al}, \mathrm{Si}_{3} \mathrm{Si}_{6} \mathrm{O}_{2}{ }_{4}\left(\mathrm{Cl}, \mathrm{CO}_{3}, \mathrm{SO}_{4}\right)\right.$

Chemie der Erde, v. 9, p. 139-144 (1934).

\section{SEFSTRÖMITE}

A mixture of ilmenite with minor amounts of radioactive minerals.

Dana VII, v. 1, p. 542.

Mineralog. Mag., v. 29, p. 112 (1950).

\section{TANTALITE}

$(\mathrm{Fe}, \mathrm{Mn})(\mathrm{Ta}, \mathrm{Cb})_{2} \mathrm{O}_{6}$

Dana VII, v. 1, p. 780-787.

Olovotantalit (or tintantalite)

Unnecessary name for stannian mangano-tantalite.

$\mathrm{U}=\mathbf{0 . 0 3}$ percent.

Am. Mineralogist, v. 46, p. 1514 (1961).

\section{TURQUOISE}

Perhaps $\mathrm{H}_{5}(\mathrm{CuOH})\left[\mathrm{Al}(\mathrm{OH})_{2}\right]_{8}\left(\mathrm{PO}_{4}\right)_{4}$

$\mathrm{U}=$ up to 0.005 percent.

Great Britain Geol. Survey Atomic Energy Div. Rept. 124, p. 1-16 (1952).

\section{VARISCITE}

$\mathrm{AlPO}_{4} \cdot 2 \mathrm{H}_{2} \mathrm{O}$

$\mathrm{U}=$ up to 0.005 percent.

Great Britain Geol. Survey Atomic Energy Div. Rept. 124, p. 1-16 (1952). 
64 GLOSSARY OF URANIUM- AND THORIUM-BEARING MINERALS

\section{WAVELLITE}

$\mathrm{Al}_{3}\left(\mathrm{PO}_{4}\right)_{2}(\mathrm{OH})_{3} \cdot 5 \mathrm{H}_{2} \mathrm{O}$

$\mathrm{U}=\mathrm{up}$ to 0.004 percent.

Heinrich, E. W., Mineralogy and geology of radioactive raw materials, 1958 , p. 130.

Fischerite

Probably is wavellite; reported to contain small amounts of $\mathrm{U}$.

Mineralog. Abs., v. 10, p. 375 (1948).

Am. Mineralogist., v. 41, p. 537-538 (1956). 


\section{INDEX}

[Valid mineral species in boldface]

A

Abernathyite.

Absite.

Abukumalite.

Adamite

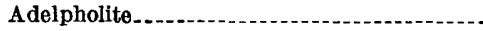

Aeschynite.

Aldanite

Allanite....................

Allophane.

Alpha-fergusonite.

Aluminobetafite

Alumo-aeschynite..........................

Alumobritholite......................................

Alvite

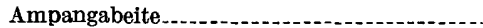

Ancylite.

Andersonite.

Anglesite

Anglesobarite.

Annerödite.

Anthraxolite

Antozonite.

Apatite

Arrhenite.

Arsenuranocircite

Arsenuranylite.

Arshinovite.

Autunite

Azorite....

Azor-pyrrhit

\section{B}

Baddeleyite.

Bagrationite

Baierite

Barite.

Bariumphosphoruranit.

Barium-phosphuranylite

Barium uranophane.

Bassetite

Bastnaesite.

Bayleyite.

Becquerelite

Bergenite.

Beta-fergusonite

Betafite

Beta-uranophane.

Beta-uranopilite

Beta-uranotile

Bilibinite

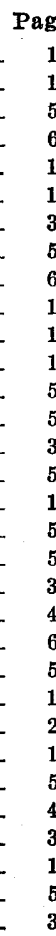

Billietite.

Page

13

Bismutomicrolite

Blomstrandine, Blomstrandinite............. 31

Blomstrandite

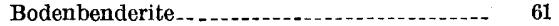

Bodenite

Boltwoodite.......... 13

Bragite_...

Brannerite_........... 13

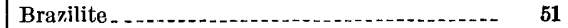

Britholite

Brockite

Bröggerite_............... 44

Broggite

Bucklandite......... 50

Buszite

Calciocarnotite........... 42

Calciogadolinite..................... 54

Calciosamarskite-............ 14

Calciotantalite

Calciothorite........... 38

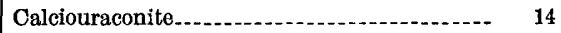

Calciovolborthite.............. 61

Calciumarsenuranit_..._._._._._._. 46

Calciumphosphoruranit_................... 11

Calciumursilite............ 46

Calcouranit_........... 11

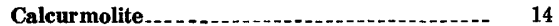

Caldasite

Calyptolite_..................... 59

Cappelenite

Carbocer................ 41

Carbonate-fluorapatite................... 51

51 Carburan..................... 41

50 Carnotite_....... 14

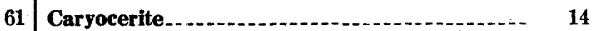

52 Cerianite_........... 14

26 Cerite................ 53

12 Cerphosphorhuttonite._._.

11 Chalcolamprite

11 Chalcolite_................................. 41

52 Chalco-uranite

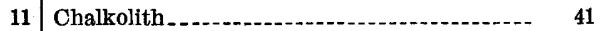

11 Cheralite

12 Chevkinite-_-_-_- 15

19 Chinglusuite_....... 53

12 Chinkolobwite

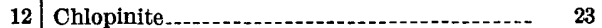

27 Chlorothorite................................ 39

13 Chrysocolla

13 Clarkeite_....... 15 


Cleveite_.
Coconinoite.
Coffinite.
Columbite.
Compreignacite.
Coracite..
Cordobaite.
Cordylite.
Corrusite.
Cousinite.
Crandallite.
Cryptolite...
Cryptomelane.
Cuprosklodowskite.
Cuprouranit...
Cuprozippeite.
Curite.s.
Cyrtolite.

\section{D}

Dakeite

Dauberite...........

\section{Davidite}

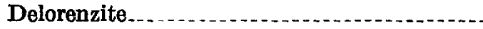

Demesmaekerite

Dewindtite.

Dianite.

Diderichite

\section{Doverite.}

Droogmansite.

Dumontite.

\section{$\mathbf{E}$}

Edwardsite

Ekanite

Eliasite

Ellsworthite

Ellweilerite

Enalite.

Endeiolite

Epidote.

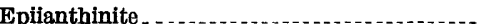

Erikite.

Eremito

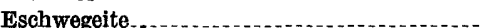

Eschynite.......................................

Eucrasite

Euxenite.

Evansite

Eytlandite.

\section{$\mathbf{F}$}

Fenghuanglite.

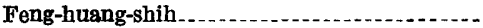
Ferghanite.

Fergusonite

Ferrocolumbite.

Ferro-llmenite

Terrothori

Fersmite.

Ferutite.

Fischerite

Florencite
Page
Fluochlore

Fluorite

Flutherite...

Formanite..._.

Fourmarierite. . .

Francevillite-_................. 19

Freyalite._......... 38

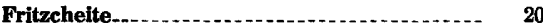

Fynchenite........ 18

Gadolinite-.................... 54

Gagarinite

Gastunite . . . .

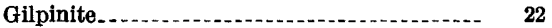

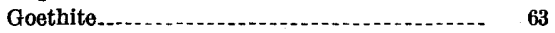

Grahamite. ................................ 41

Grayite

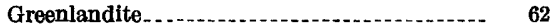

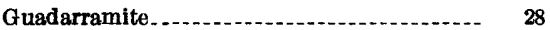

Guilleminite............ 20

Gummite.......................... 20

Haddamite $\ldots . . . . . . . . . . . . . . .27$

Hagatalite............ 59

Haiweeite ............. 20

Hallimondite.

Hatchettolite................................ 32

Heinrichite_................................. 21

Hellandite-.............. 54

Hermannolite....

Hibonite.

Hielmite... . .

Hjelmite............. 21

Hlopinite_. . . .

Hoegtveitite........... 59

Hokutolite

Hügelite.

Huttonite

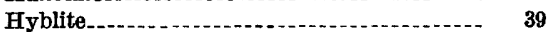

Hydrocerite.....

Hydrochlore ... . .

Hydroeuxenite........ 34

Hydrogen autunite........................ 21

Hydrothorite

Hydronasturan................ 22

Hydrosamarskite............... 34

Hydroxyl-bastnaesite........ 52

Hydroxyl-thorite ...................... 39

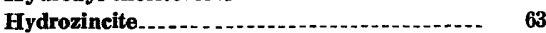

Ianthinite.

Idocrase

Iriginite

Irinite................ 55

Iron uranite

Ishikawaite......... 22

Jáchymovite

Jiningite-1. 38

Johannite- . -

Johnstrupite-(nt 
Kahlerite

24 Meta-uranopilite _........................ 26

Kalk-uran-carbonat.........................

Karnasurtite

Kasolite.

Katangite

Khlopinite

Kieselkupfer-uranoxyd

Kivuite.

Kobeite

Kochelite

Kolm

Koppite

Korarfveite

Kozhanovite.

Kupferarsenuranit

Kupferphosphoruranit.

\section{L}

Lambertite

Lermontovite.

Liebigite

Lime-uranite

Limonite

Lodochnikite

Loparite.

Lovchorrite

Lovozerite.

Lueshite.

Lyndochite.

\section{M}

Mackelveyite

Mackintoshite

Magnesium-phosphoruranit

Magnesium ursilite

Maitlandite.

Malacon

Manganocolumbite

Manganosteenstrupine

Marignacite.

Masuyite

Mavudzite..

McKelveyite -

Medjidite

Melanocerite.

Mendeleyevite

Mengite

Meta-ankoleïte

Meta-autunite I

Meta-autunite II.

Metachalcolite.

Metahaiweeite.

Metaheinrichite

Metakahlerite.

Metakalkuranite..

Metakirchheimerite

Metakupieruranit.

Metanovacekite

Metasalpéeite

Metasandbergerite.

Metaschoepite.

Metasimpsonite

Metatorbernite.

Metatyuyamunite

Meta-uranocircite

Naegite

Meta-uranospinite-- 27

Metavandendriesscheite..................... 47

Metazellerite............... 27

Metazeunerite........ 27

Microlite_.............. 27

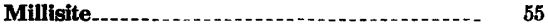

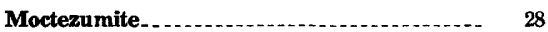

Moluranite_..._.

Monazite......................... 28

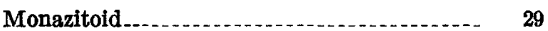

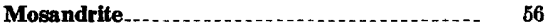

Mourite.......... 29

Mozambikite................. 38

Muromontite

Nagatelite.....

Nasturan

Natroautunite. ........ 29

Natroniobite............ 56

Nenadkevite.

Neogastunite................. 36

Neotantalite................................ 28

Nicolayite............................. 40

Ningyoite.......... 29

Niobo-aeschynite

Nioboloparite......

Niobozirconolite.............................. 60

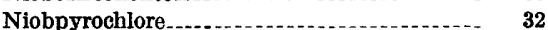

Niobtantalpyrochlore......................... 28

Nivenite_............ 45

Nohlite.............. 30

Novacekite. . . . .

Nuevite....

Nuolaite

Obruchevite-1... 30

Oerstedite................ 60

Oliveiraite_......... 18

Olovotantalit................................. 63

Opal

Orangite-........... 38

Orlite.............. 30

Orthite_... 50

Orthochevkinite.................. 30

Oyamalite...... 60

Pallite-_._.

Pandaite...

Para-autunite..........

Parapitchblende............ 30

Paraschoepite................ 35

Parisite......... 56

Parsonsite ............. 30

Paulite

Peligotite_-_._.

Perrierite-........ 31

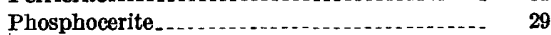

Phosphuranylite.................. 31

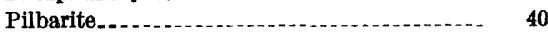

Pisekite_.......... 31 


Pitchblende.
Pittinite.
Plumboniobite.
Polycrase.
Powellite.
Pravdite.
Priazovite.
Priorite...
Prjevalskite.
Przhevalskite.
Pseudo-autunite.
Pseudowavellite.
Pyrochlore.
Pyromorphite.
Pyrrbite...

\section{$\mathbf{R}$}

Rabbittite.

Radiobarite...

Randite.

Ranquilite.

Rauvite.

Renardite

Rhabdophane

Ribeirite

Richetite

Rijkeboerite

Rinkite..... . . . .

Rinkolite

Risörite-

Rogersite

Rowlandite

Rutherfordine

Rutherfordite.

\section{S}

Sabugalite

\section{Saléite}

Samarskite

Samiresite

Sandbergerite

Saryarkite

Seapolite

Schoepite.

Schroeckingerite

Sedovite.

Sengierite

Sharpite

Shentulite.

Shinkolobwite

Silicorhabdophane

Silicosmirnovskite

Sinicite

Sipylite

Sklodovskite

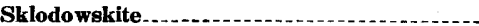

Smirnovite

Smirnovskite

Soda-autunite

Soddite.

Sodium autunite

Sodium uranospinite
Page

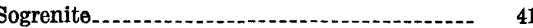

Spencite-.........- 57

Sphene-_._.

Stasite.... . - 17

Steenstrupine. . . .

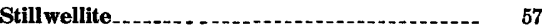

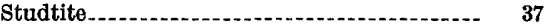

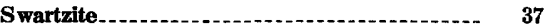

Synchysite... .

\section{$\mathbf{T}$}

Tachyaphaltite................................ 60

Tangeite, Tangueite_................. 61

Tantalite........................... 63

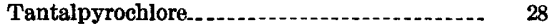

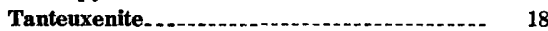

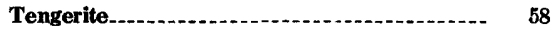

Thalenite

Thorbastnaesite_............. 37

Thorianite.................. 37

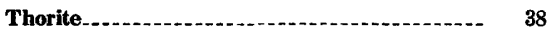

Thoro-aeschynite-_...

Thorogummite_................. 39

Thorosteenstrupine

Thorotungstite............................... 59

Thorutite. . .

Thucholite_............ 40

Tintantalite

Titanite......... .57

Titanoniobite. ......-...-. 18

Titanorhabdophane........... 58

Titanothucholite........ 41

Toddite_............. 35

Torberite........................................ 41

Torbernite-............... 41

Torutite

Treanorite_....... 50

Tritomite-_._.

Troegerite-................ 42

Tscheffkinite..................... 15

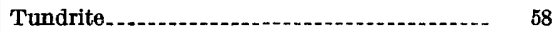

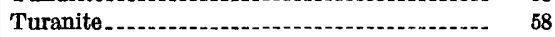

Turnerite

Turquoise.

Tyrite-_._- 19

Tyuyamunite._._............ 42

Ufertite............. 16

Ulrichite......... 45

Umohoite

Unnamed mineral(s) of -

Antipov

Belova and others. 42

Chernik......... 43

Chirvinsky.................................. 43

Frondel .

Melkov - .

Protas

Soboleva and Pudovkina..... 43

TAte district of Mozambique............ 17

Walenta............................................. 44

Uraconite.............. 44

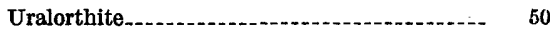

Uramphite_....................... 44 
Uran-apatite

Page

Page

Uranatemnite

Voglite

Uraninite

Uranite

Vudyavrite

Uran-Kalk-Kupter-C

Uran-mica

Uranochalcite

Uranocher

Uranocircite.

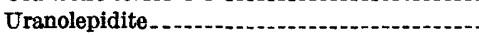

Uranoniobite.

Uranophane.

Uranphyllit.

Uranopilite.

Uranopissite

Uranospathite

Uranosphaerite, Uranospherite

Uranospinite

Uranotantalite.

Walpurgite.

\section{W}

Uranothallite

Uranothorianite.........................................

Uranothorite.

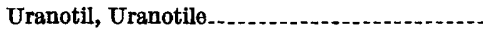

Uranphyllit

Uranpyrochlore

Uranvitriol

Urdite.

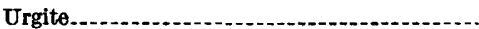

Urhyte.

Ursilite

Usigite, Usihyte.

Uvanite.

Waltherite._..................................... 48

Wasite............. 51

Wavellite........................... 64

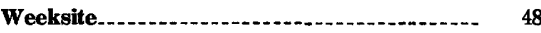

Widenmannite........... 48

Wiikite_.............. 48

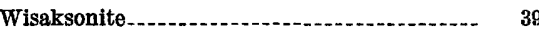

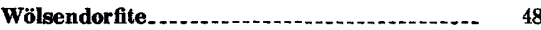

Wulfenite................... 58

Wyartite....... 48

Xanthorite_................... 51

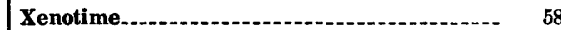

Vandenbrandeite

Yamagutilite, Yamaguchilite

Yanshainshynite..................... 39

Yttrialite........................................ 49

Yttrium orthite

Yttrobetafite.......... 30

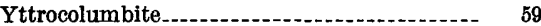

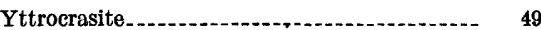

Yttrogummite.............. 20

Yttro-ilmenite_.................................. 35

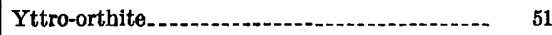

Yttrotantalite................................ 59

Yttrotungstite_...

Vandendriegscheite

Vanoxite.

Vanuralite..........................................

Vanuranylite.

Variseite.

Vesuvianite.

Vietinghofite.

Voglianite.

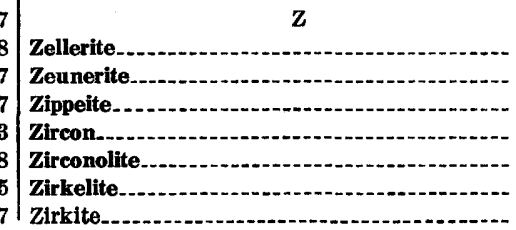






